\title{
KNOWLEDGE REPRESENTATION WITH LOGIC PROGRAMS
}

\author{
Gerhard Brewka and Jürgen Dix
}

\begin{abstract}
In this tutorial-overview, which resulted from a lecture course given by the authors at the European Summer School in Logic, Language and Information 1997 in Aix-en-Provence (http: //ww.lpl.univ-aix.fr/ "esslli97/), we show how knowledge representation (KR) can be done with the help of generalized logic programs. We start by introducing the core of PROLOG, which is based on definite logic programs. Although this class is very restricted (and will be enriched by various additional features in the rest of the paper), it has a very nice property for KR-tasks: there exist efficient query-answering procedures - a top-down approach and a bottom-up evaluation. In addition we can not only handle ground queries but also queries with variables and compute answer-substitutions.

It turns out that more advanced KR-tasks can not be properly handled with definite programs. Therefore we extend this basic class of programs by additional features like negation-as-finite-failure, defaultnegation, explicit negation, preferences, and disjunction. The need for these extensions is motivated by suitable examples and the corresponding semantics are discussed in detail.

Clearly, the more expressive the respective class of programs under a certain semantics is, the less efficient are potential query-answering methods. This point will be illustrated and discussed for every extension. By well-known recursion-theoretic results, it is obvious that there do not exist complete query-answering procedures for the general case where variables and function symbols are allowed. Nevertheless we consider it an important topic of further research to extract feasible classes of programs where answer-substitutions can be computed.
\end{abstract}

\section{INTRODUCTION}

One of the major reasons for the success story (if one is really willing to call it a success story) of human beings on this planet is our ability to invent tools that help us improve our-otherwise often quite limited—capabilities. The invention of machines that are able to do interesting things, like transporting people from one place to the other (even through the air), sending moving pictures and sounds around the globe, bringing our email to the right person, and the like, is one of the cornerstones of our culture and determines to a great degree our everyday life.

Among the most challenging tools one can think of are machines that are able to handle knowledge adequately. Wouldn't it be great if, instead of the stupid device which brings coffee from the kitchen to your offi ce every day at 9 am, and which needs complete reengineering whenever your coffee preferences change, you could (for the same price, admitted) get a smart robot who simply can be told that you want your coffee black this morning, and that you need an extra Aspirin since it was your colleague's birthday yesterday? To react in the right way to your needs such a robot would have to know a lot, for instance that Aspirin should come with a glass of water, or that people in certain situations need their coffee extra strong.

Building smart machines of this kind is at the heart of Artifi cial Intelligence (AI). Since such machines will need tremendous amounts of knowledge to work 
properly, even in very limited environments, the investigation of techniques for representing knowledge and reasoning is highly important.

In the early days of $\mathrm{AI}$ it was still believed that modeling general purpose problem solving capabilites, as in Newell and Simon's famous GPS (General Problem Solver) program, would be suffi cient to generate intelligent behaviour. This hypothesis, however, turned out to be overly optimistic. At the end of the sixties people realized that an approach using available knowledge about narrow domains was much more fruitful. This led to the expert systems boom which produced many useful application systems, expert system building tools, and expert system companies. Many of the systems are still in use and save companies millions of dollars per year ${ }^{1}$.

Nevertheless, the simple knowledge representation and reasoning methods underlying the early expert systems soon turned out to be insuffi cient. Most of the systems were built based on simple rule languages, often enhanced with ad hoc approaches to model uncertainty. It became apparent that more advanced methods to handle incompleteness, defeasible reasoning, uncertainty, causality and the like were needed.

This insight led to a tremendous increase of research on the foundations of knowledge representation and reasoning. Theoretical research in this area has blossomed in recent years. Many advances have been made and important results were obtained. The technical quality of this work is often impressive.

On the other hand, most of these advanced techniques have had surprisingly little influence on practical applications so far. To a certain degree this is understandable since theoretical foundations had to be laid fi rst and pioneering work was needed. However, if we do not want research in knowledge representation to remain a theoreticians' game more emphasis on computability and applicability seems to be needed. We strongly believe that the kind of research presented in this tutorial, that is research aiming at interesting combinations of ideas from logic programming and nonmonotonic reasoning, provides an important step into this direction.

\subsection{Some History}

Historically, logic programs have been considered in the logic programming community for more than 20 years. It began with [Colmerauer et al., 1973; Kowalski, 1974; van Emden and Kowalski, 1976] and led to the defi nition and implementation of $P R O L O G$, a by now theoretically well-understood programming language (at least the declarative part consisting of Horn-clauses: pure PROLOG). Extensions of PROLOG allowing negative literals have been also considered in this area: they rely on the idea of negation-as-fi nite-failure, we call them logicprogramming-semantics (or shortly LP-semantics).

\footnotetext{
${ }^{1}$ We refer the interested reader to [Russel and Norvig, 1995] which gives a very detailed and nice exposition of what has been done in AI since its very beginning until today.
} 
In parallel, starting at about 1980, Nonmonotonic Reasoning entered into computer science and began to constitute a new fi eld of active research. It was originally initiated because Knowledge Representation and Common-Sense Reasoning using classical logic came to its limits. Formalisms like classical logic are inherently monotonic and they seem to be too weak and therefore inadequate for such reasoning problems.

In recent years, independently of the research in logic programming, people interested in knowledge representation and nonmonotonic reasoning also tried to defi ne declarative semantics for programs containing default or explicit negation and even disjunctions. They defi ned various semantics by appealing to (different) intuitions they had about programs.

This second line of research started in 1986 with the Workshop on the Foundations of Deductive Databases and Logic Programming organized by Jack Minker: the revised papers of the proceedings were published in [Minker, 1988]. The stratifi ed (or the similar perfect) semantics presented there can be seen as a splittingpoint: it is still of interest for the logic programming community (see [Cavedon and Lloyd, 1989]) but its underlying intuitions were inspired by nonmonotonic reasoning and therefore much more suitable for knowledge representation tasks. Semantics of this kind leave the philosophy underlying classical logic programming in that their primary aim is not to model negation-as-fi nite-failure, but to construct new, more powerful semantics suitable for applications in knowledge representation. Let us call such semantics NMR-semantics.

Nowadays, due to the work of Apt, Blair and Walker, Fitting, Lifschitz, Przymusinski and others, very close relationships between these two independent research lines became evident. Methods from logic programming, e.g. least fi xpoints of certain operators, can be used successfully to defi ne NMR-semantics.

The NMR-semantics also shed new light on the understanding of the classical nonmonotonic logics such as Default Logic, Autoepistemic Logic and the various versions of Circumscription. In addition, the investigation of possible semantics for logic programs seems to be useful because

1. parts of nonmonotonic systems (which are usually defi ned for full predicate logic, or even contain additional (modal)-operators) may be "implemented" with the help of such programs,

2. nonmonotonicity in these logics may be described with an appropriate treatment of negation in logic programs.

\subsection{Non-Monotonic Formalisms in KR}

As already mentioned above, research in nonmonotonic reasoning has begun at the end of the seventies. One of the major motivations came from reasoning about actions and events. John McCarthy and Patrick Hayes had proposed their situation calculus as a means of representing changing environments in logic. The basic idea is to use an extra situation argument for each fact which describes the situation in 
which the fact holds. Situations, basically, are the results of performing sequences of actions. It soon turned out that the problem was not so much to represent what changes but to represent what does not change when an event occurs. This is the so-called frame problem. The idea was to handle the frame problem by using a default rule of the form

If a property $P$ holds in situation $S$ then $P$ typically also holds in the situation obtained by performing action $A$ in $S$.

Given such a rule it is only necessary to explicitly describe the changes induced by a particular action. All non-changes, for instance that the real colour of the kitchen wall does not change when the light is turned on, are handled implicitly. Although it turned out that a straightforward formulation of this rule in some of the most popular nonmonotonic formalisms may lead to unintended results the frame problem was certainly the challenge motivating many people to join the fi eld.

In the meantime a large number of different nonmonotonic logics have been proposed. We can distinguish four major types of such logics:

1. Logics using nonstandard inference rules with an additional consistency check to represent default rules. Reiter's default logic (see Appendix A.3) and its variants are of this type.

2. Nonmonotonic modal logics using a modal operator to represent consistency or (dis-) belief. These logics are nonmonotonic since conclusions may depend on disbelief. The most prominent example is Moore's autoepistemic logic.

3. Circumscription (see Appendix A.4) and its variants. These approaches are based on a preference relation on models. A formula is a consequence iff it is true in all most preferred models of the premises. Syntactically, a second order formula is used to eliminate all non-preferred models.

4. Conditional approaches which use a non truth-functional connective $\sim$ to represent defaults. A particularly interesting way of using such conditionals was proposed by Kraus, Lehmann and Magidor. They consider $p$ as a default consequence of $q$ iff the conditional $q \sim p$ is in the closure of a given conditional knowledge base under a collection of rules. Each of the rules directly corresponds to a desirable property of a nonmonotonic inference relation.

The various logics are intended to handle different intuitions about nonmonotonic reasoning in a most general way. On the other hand, the generality leads to problems, at least from the point of view of implementations and applications. In the fi rst order case the approaches are not even semi-decidable since an implicit consistency check is needed. In the propositional case we still have tremendous complexity problems. For instance, the complexity of determining whether a formula is contained in all extensions of a propositional default theory is on the second 
level of the polynomial hierarchy. As mentioned earlier we believe that logic programming techniques can help to overcome these diffi culties.

Originally, nonmonotonic reasoning was intended to provide us with a fast but unsound approximation of classical reasoning in the presence of incomplete knowledge. Therefore one might ask whether the higher complexity of NMRformalisms (compared to classical logic) is not a real drawback of this aim? The answer is that NMR-systems allow us to formulate a problem in a very compact way as a theory $T$. It turns out that for some problems any equivalent formulation in classical logic (if possible at all) as a theory $T^{\prime}$ is much larger: the size of $T^{\prime}$ is exponential in the size of $T$ ! We refer to [Gogic et al., 1995] and [Cadoli et al., 1996; Cadoli et al., 1997; Cadoli et al., 1995] where such problems are investigated.

\subsection{How this Paper is organized}

In this overview paper we show how Knowledge Representation can be done with the help of generalized logic programs. We start by introducing the core of PROLOG, which is based on defi nite logic programs. Although this class is very restricted (and will be enriched by various additional features in the rest of the paper), it has a very nice property for KR-tasks: there exist effi cient query-answering procedures - a top-down approach and a bottom-up evaluation. In addition we can not only handle ground queries but also queries with variables and compute answer-substitutions.

It turns out that more advanced KR-tasks can not be properly handled with definite programs. Therefore we extend this basic class of programs by additional features like negation-as-fi nite-failure, default-negation, explicit negation, preferences, and disjunction. The need for these extensions is motivated by suitable examples and the corresponding semantics are also discussed.

Clearly, the more expressive the respective class of programs under a certain semantics is, the less effi cient are potential query-answering methods. This point will be illustrated and discussed for every extension. By well-known recursiontheoretic results, it is obvious that there do not exist complete query-answering procedures for the general case where variables and function symbols are allowed. Nevertheless we consider it an important topic of further research to extract feasible classes of programs where answer-substitutions can be computed.

\section{DEFINITE LOGIC PROGRAMS}

In this section we consider the most restricted class of programs: defi nite logic programs, programs without any negation at all. All the extensions of this basic class we will introduce later contain at least some kind of negation (and perhaps additional features). But here we also allow the ocurrence of free variables as well as function symbols. 
In Section 2.1 we introduce as a representative for the top-down approach the SLD-resolution. Section 2.2 presents the main competing approach of SLD: bottomup evaluation. This approach is used in the database community and it is effi cient when additional assumptions are made ( $f$ niteness-assumptions, no function symbols). In Section 2.3 we consider the influence and appropriateness of Herbrand models and their underlying intuition. Finally in Section 2.4 we present and discuss two important examples in KR: reasoning in inheritance hierarchies and reasoning about actions. Both examples clearly motivate the need of extending defi nite programs by a kind of default-negation "not".

First some notation used throughout this paper. A language $\mathcal{L}$ consists of a set of relation symbols and a set of function symbols (each symbol has an associated arity). Nullary functions are called constants. Terms and atoms are built from $\mathcal{L}$ in the usual way starting with variables, applying function symbols and relationsymbols.

Instead of considering arbitrary $\mathcal{L}$-formulae, our main object of interest is a program:

Definition 2.1 (Definite Logic Program).

A defi nite logic program consists of a fi nite number of rules of the form

$$
A \leftarrow B_{1}, \ldots, B_{m}
$$

where $A, B_{1}, \ldots, B_{m}$ are positive atoms (containing possibly free variables). We call $A$ the head of the rule and $B_{1}, \ldots, B_{m}$ its body. The comma represents conjunction $\wedge$.

We can think of a program as formalizing our knowledge about the world and how the world behaves. Of course, we also want to derive new information, i.e. we want to ask queries:

\section{Definition 2.2 (Query).}

Given a defi nite program we usually have a defi nite query in mind that we want to be solved. A defi nite query $Q$ is a conjunction of positive atoms $C_{1} \wedge \ldots \wedge C_{l}$ which we denote by

$$
\text { ?- } C_{1}, \ldots, C_{l} \text {. }
$$

These $C_{i}$ may also contain variables. Asking a query $Q$ to a program $P$ means asking for all possible substitutions $\Theta$ of the variables in $Q$ such that $Q \Theta$ follows from $P$. Often, $\Theta$ is also called an answer to $Q$. Note that $Q \Theta$ may still contain free variables.

Note that if a program $P$ is given, we usually assume that it also determines the underlying language $\mathcal{L}$, denoted by $\mathcal{L}_{P}$, which is generated by exactly the symbols ocurring in $P$. The set of all these atoms is called the Herbrand base and denoted by $B_{\mathcal{L}_{P}}$ or simply $B_{P}$. The corresponding set of all ground terms is the Herbrand universe. Another important notion that we are not explaining in detail here is that of unifi cation. Given two atoms $A$ and $B$ with free variables we can ask if we can 
compute two substitutions $\Theta_{1}, \Theta_{2}$ for the variables such that

$$
A \Theta_{1} \text { is identical to } B \Theta_{2} \text {, }
$$

or if we can decide that this is not possible at all. In fact, if the two atoms are unifi able we can indeed compute a most general unifi er, called mgU (see[Lloyd, 1987]). The $\mathrm{mgU} \Theta$ is a substitution defi ned on the set of variables occurring in both $A$ and $B$ such that $A \Theta$ is identical to $B \Theta$.

This will be important in our framework because if an atom appears as a subgoal in a query, we may want to determine if there are rules in the program whose heads unify with this atom.

How are our programs related to classical predicate logic? Of course, we can map a program-rule into classical logic by interpreting " $\leftarrow$ " as material implication " $\supset$ " and universally quantifying. This means we view such a rule as the following universally quantifi ed formula

$$
B_{1} \wedge \ldots \wedge B_{m} \supset A \text {. }
$$

However, as we will see later, there is a great difference: a logic program-rule takes some orientation with it. This makes it possible to formulate the following principle as an underlying intuition of all semantics of logic programs:

\section{Principle 1 (Orientation).}

If a ground atom $A$ does not unify with some head of a program rule of $P$, then this atom is considered to be false. In this case we say that "not $A$ " is derivable from $P$ to distinguish it from classical $\neg A$.

The orientation principle is nothing but a weak form of negation-by-failure. Given an intermediate goal not $A$, we fi rst try to prove $A$. But if $A$ does not unify with any head, $A$ fails and this is the reason to derive not $A$.

\subsection{Top-Down}

SLD-Resolution ${ }^{2}$ is a special form of Robinson's general resolution rule. While Robinson's rule is complete for full fi rst order logic, SLD is complete for defi nite logic programs (see Theorem 2.1 on page 9). We do not give a complete defi nition of SLD-resolution (see [Lloyd, 1987]) but rather prefer to illustrate its behaviour on the following example.

\section{Example 2.1 (SLD-Resolution).}

Let the program $P_{S L D}$ consist of the following three clauses

(1) $p(x, z) \leftarrow q(x, y), p(y, z)$

(2) $p(x, x)$

(3) $q(a, b)$

${ }^{2}$ SL-resolution for Definite clauses. SL-resolution stands for Linear resolution with Selection function. 


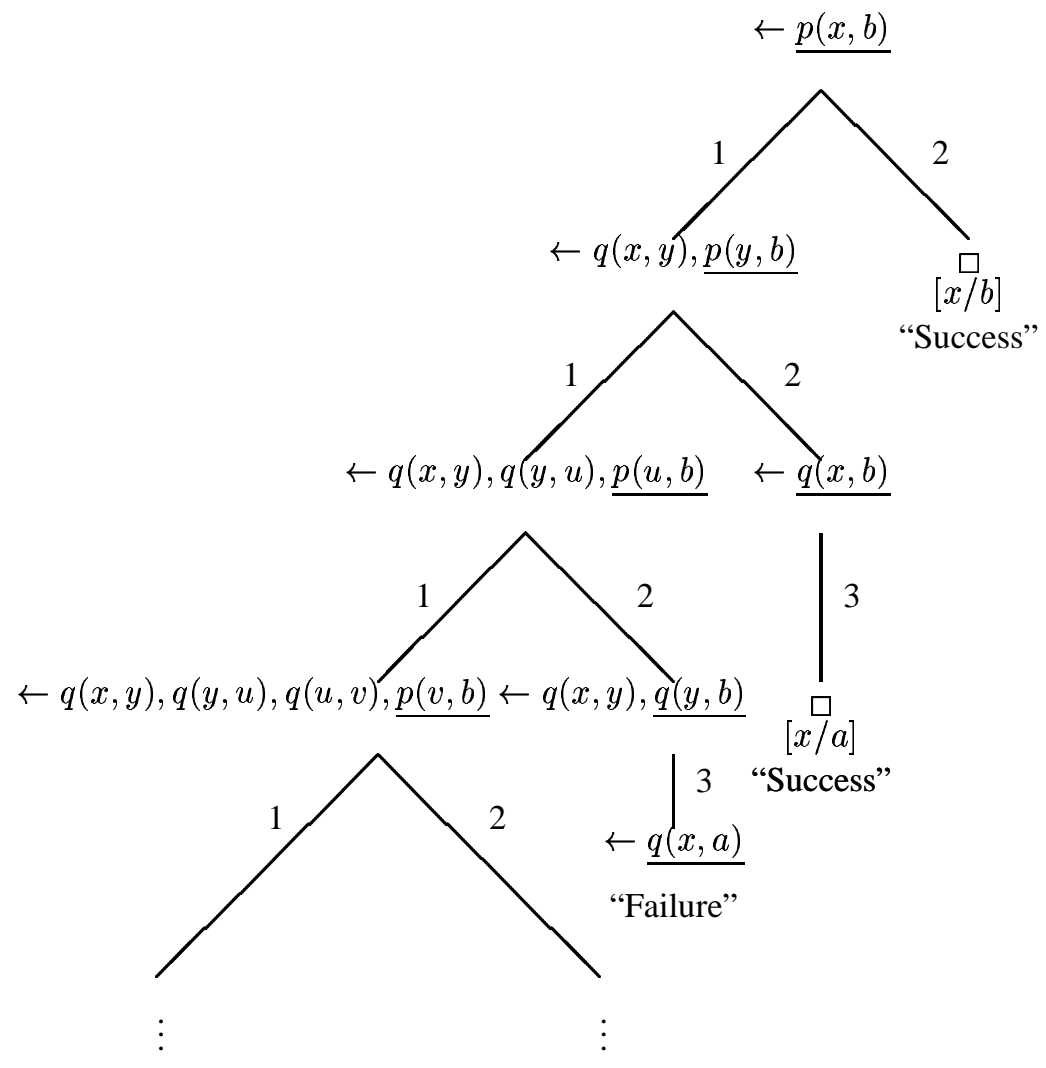

Figure 1. An infi nite SLD-Tree

The query $Q$ we are interested in is given by $p(x, b)$. I.e. we are looking for all substitutions $\Theta$ for $x$ such that $p(x, b) \Theta$ follows from $P$.

Figure 1 illustrates the behaviour of SLD-resolution. We start with our query in the form $\leftarrow Q$. Sometimes the notation $\square \leftarrow Q$ is also used, where $\square$ denotes the falsum. In any round the selected atom is underlined: numbers 1,2 or 3 indicate the number of the clause which the selected atom is resolved against. Obviously, there are three different sorts of branches, namely

1. infi nite branches,

2. branches that end up with the empty clause, and

3. branches that end in a deadlock ("Failure"): no applicable rule is left.

In this example we always resolve with the last atom in the goal under consideration. If we choose always the $f i r s t$ atom in the goal, we will obtain, at least in this example, a fi nite tree. 
Defi nite programs have the nice feature that the intersection of all Herbrandmodels exists and is again a Herbrand model of $P$. It is denoted by $M_{P}$ and called the least Herbrand-model of $P$. Note that our original aim was to fi nd substitutions $\Theta$ such that $Q \Theta$ is derivable from the program $P$. This task as well as $M_{P}$ is closely related to SLD:

\section{Theorem 2.1 (Soundness and Completeness of SLD).}

The following properties are equivalent:

- $P=\forall Q \Theta$, i.e. $\forall Q \Theta$ is true in all models of $P$,

- $M_{P}=\forall Q \Theta$,

- SLD computes an answer $\tau$ that subsumes ${ }^{3} \Theta$ wrt $Q$.

Note that not any correct answer is computed, only the most general one is (which of course subsumes all the correct ones).

The main feature of SLD-resolution is its goal-orientedness. SLD automatically ensures (because it starts with the Query) that we consider only those rules that are relevant for the query to be answered. Rules that are not at all related are simply not considered in the course of the proof.

\subsection{Bottom-Up}

We mentioned in the last section the least Herbrand model $M_{P}$. The bottom-up approach can be described as computing this least Herbrand model from below. We start fi rst with rules with empty bodies (in our example these are all instantiations of rules (2) and (3)). We get as facts all atoms that are in the heads of rules with empty bodies (namely $p(a, a), p(b, b), q(a, b)$ in Example 2.1 on page 7). In the next round we use the facts that we computed before and try to let the rules "fi re", i.e. when their bodies are true, we add their heads to the atoms we already have (this gives us $p(a, b)$ ).

To be more precise we introduce the immediate consequence operator $T_{P}$ which associates to any Herbrand model another Herbrand model.

Example 2.2 $\left(T_{P}\right)$.

Given a defi nite program $P$ let $T_{P}: 2^{B_{P}} \longmapsto 2^{B_{P}} ; \mathcal{I} \longmapsto T_{P}(\mathcal{I})$

$$
\begin{aligned}
T_{P}(\mathcal{I}):=\left\{A \in B_{P}:\right. & \text { there is an instantiation of a rule in } P \\
& \text { s.t. } A \text { is the head of this rule and all } \\
& \text { body-atoms are contained in } \mathcal{I}\}
\end{aligned}
$$

It turns out that $T_{P}$ is monotone and continuous so that (by a general theorem of Knaster-Tarski) the least fi xpoint is obtained after $\omega$ steps. Moreover we have

${ }^{3}$ i.e. $\exists \sigma: Q \tau \sigma=Q \Theta$. 
Theorem $2.2\left(T_{P}\right.$ and $\left.M_{P}\right)$. $M_{P}=T_{P} \uparrow^{\omega}=l f p\left(T_{P}\right)$.

This approach is especially important in database applications, where the underlying language does not contain function symbols (DATALOG) - this ensures the Herbrand universe to be fi nite. Under this condition the iteration stops after fi nitely many steps. In addition, rules of the form

$$
p \leftarrow p
$$

do not make any problems. They simply can not be applied or do not produce anything new. Note that in the top-down approach, such rules give rise to infi nite branches! Later, elimination of such rules will turn out to be an interesting property. We therefore formulate it as a principle:

\section{Principle 2 (Elimination of Tautologies).}

Suppose a program $P$ has a rule which contains the same atom in its body as well as in its head (i.e. the head consists of exactly this atom). Then we can eliminate this rule without changing the semantics.

Unfortunately, such a bottom-up approach has two serious shortcomings. First, the goal-orientedness of SLD-resolution is lost: we are always computing the whole $M_{P}$, even those facts that have nothing to do with the query. The reason is that in computing $T_{P}$ we do not take into account the query we are really interested in. Second, in any step facts that are already computed before are recomputed again. It would be more effi cient if only new facts were computed. Both problems can be (partially) solved by appropriate refi nements of the naive approach:

- Semi-naive bottom-up evaluation ([Bry, 1990; Ullman, 1989a]),

- Magic Sets techniques ([Beeri and Ramakrishnan, 1991; Ullman, 1989b]).

\subsection{Herbrand-Models and the underlying language}

Usually when we represent some knowledge in fi rst order logic or even in logic programs, it is understood that the underlying language is given exactly by the symbols that occur in the formal theory. Suppose we have represented some knowledge about the world as a theory $T$ in a language $\mathcal{L}$. Classical predicate logic formalizes the notion of a formula $\phi$ entailed by the theory $T$. This means that $\phi$ is true in all models of $T$ (we denote this set by $\operatorname{MOD}(T)$ ). Why are we considering all models? Doesn't it make sense to look only at Herbrand models, i.e. to models generated by the underlying language? After all we are not interested in models that contain elements which are not representable as terms in our language. These requirements are usually called unique names assumption and domain closure assumption:

\section{Definition 2.3 (UNA and DCA).}

Let a language $\mathcal{L}$ be given. We understand by the unique names assumption the 
restriction to those models $\mathcal{I}$, where syntactically different ground $\mathcal{L}$-terms $t_{1}, t_{2}$ are interpreted as nonidentical elements: $t_{1}^{\mathcal{I}}$ is not identical to $t_{2}^{\mathcal{I}}$.

By the domain closure assumption we mean the restriction to those models $\mathcal{I}$ where for any element $a$ in $\mathcal{I}$ there is a $\mathcal{L}$-term $t$ that represents this element: $a=t^{\mathcal{I}}$.

As an example, in Theorem 2.1 on page 9 of Section 2.1 we referred to $M_{P}$, the least Herbrand model of $P$. The reason that the fi rst equivalence in this theorem holds is given by the fact that for universal theories $T$ and existential formulae $\phi$ the following holds

$$
\operatorname{MOD}(T) \models \phi \text { iff } \operatorname{Herb}_{\mathcal{L}^{-}} \operatorname{MOD}(T) \models \phi .
$$

In our particular case, where $T$ is a defi nite program $P$, we can even replace $\operatorname{Herb}_{\mathcal{L}}-\operatorname{MOD}(T)$ in the above equation by the single model $M_{P}$.

This last result does not hold in general. But what happens if we nevertheless are interested in only the Herbrand-models of a theory $T$ (and therefore automatically ${ }^{4}$ assume UNA and DCA)? At fi rst sight one can argue that such an approach is much simpler: in contrast to all models we only need to take care about the very specifi c Herbrand models. But it turns out that determining the truth of a formula in all Herbrand models is a much more complex task (namely $\Pi_{1}^{1}$-complete) than to determine if it is true in all models. This latter task is also undecidable in general, but it is recursively enumerable, i.e. $\Pi_{1}^{0}$-complete. The fact that this task is recursively enumerable is the content of the famous completeness theorem of Gödel, where "truth of a formula in all models" is shown to be equivalent to deriving this formula in a particular axiomatization of the predicate calculus of fi rst order. We refer to the appendix (Section A.1 and Section A.2) where the necessary notions are introduced.

But we have still a problem with Theorem 2.1 on page 9 in our restricted setting:

\section{Example 2.3 (Universal Query Problem).}

Consider the program $P:=p(a)$, the query $Q:=p(x)$ and the empty substitution $\Theta:=\epsilon$. We have

- $M_{P}=\forall x p(x)$,

- but SLD only computes the answer $x / a$.

Przymusinski called this the universal query problem.

There are essentially two solutions to avoid this behaviour: to use a language which is rich enough (i.e. contains suffi ciently many terms, not only those ocurring in the program $P$ itself) or to consider arbitrary models, not only Herbrand models. Both approaches have been followed in the literature but they are beyond the scope of this paper.

\footnotetext{
${ }^{4}$ The only difference between Herbrand models and models satisfying UNA and DCA is that the interpretation of terms is uniquely determined in Herbrand models. It is required that a term " $f\left(t_{1}, \ldots, f_{n}\right)$ " is interpreted in a Herbrand model $\mathcal{I}$ as " $f\left(\mathcal{I}\left(t_{1}^{\mathcal{I}}, \ldots, t_{n}^{\mathcal{I}}\right)\right.$ ".
} 


\subsection{Why going beyond Defi nite Programs?}

So far we have a nice query-answering procedure, SLD-Resolution, which is goaloriented as well as sound and complete with respect to general derivability. But note that up to now we are not able to derive any negative information. Not even our queries allow this. From a very pragmatic viewpoint, we can consider " not $A$ " to be derivable if $A$ is not. Of course, this is not sound with respect to classical logic but it is with respect to $M_{P}$.

In KR we do not only want to formulate negative queries, we also want to express default-statements of the form

Normally, unless something abnormal holds, then $\psi$ implies $\phi$.

Such statements were the main motivation for nonmonotonic logics, like Default Logic or Circumscription (see Section A.3 and Section A.4 of the appendix). How can we formulate such a statement as a logic program? The most natural way is to use negation " not"

$$
\phi \leftarrow \psi, \operatorname{not} a b
$$

where $a b$ stands for abnormality. Obviously, this forces us to extend defi nite programs by negative atoms, we call them default atoms.

A typical example for such statements occurs in Inheritance Reasoning. We take the following example from [Baral and Gelfond, 1994]:

\section{Example 2.4 (Inheritance Hierachies).}

Suppose we know that birds typically fly and penguins are non-flying birds. We also know that Tweety is a bird. Now an agent is hired to build a cage for Tweety. Should the agent put a roof on the cage? After all it could be still the case that Tweety is a penguin and therefore can not fly, in which case we would not like to pay for the unneccessary roof. But under normal conditions, it should be obvious that one should conclude that Tweety is flying.

A natural axiomatization is given as follows:

$$
\begin{aligned}
& P_{\text {Inheritance }}: \operatorname{flies}(x) \leftarrow \operatorname{bird}(x), \quad \operatorname{not} a b\left(r_{1}, x\right) \\
& \operatorname{bird}(x) \leftarrow \operatorname{penguin}(x) \\
& a b\left(r_{1}, x\right) \leftarrow \operatorname{penguin}(x) \\
& \text { make_top }(x) \leftarrow \operatorname{flies}(x)
\end{aligned}
$$

together with some particular facts, like e.g. bird(Tweety) and penguin(Sam). The fi rst rule formalizes our default knowledge, while the third formalizes that the default rule should not be applied in abnormal or exceptional cases. In our example, it expresses the famous specifi city principle which says that more specifi c knowledge should override more general one ([Touretzky, 1986; Touretzky et al., 1988; Horty et al., 1990]).

For the query "make_top(Tweety)" we expect the answer "yes" while for "make_top(Sam)" we expect the answer "no". 
Another important KR task is to formalize knowledge for reasoning about action. We again consider a particular important instance of such a task, namely temporal projection. The overall framework consists in describing the initial state of the world as well as the effects of all actions that can be performed. What we want to derive is how the world looks like after a sequence of actions has been performed.

\section{Example 2.5 (Temporal Projection: Yale-Shooting Problem).}

We distinguish between three sorts ${ }^{5}$ of variables:

- situation variables: $S, S^{\prime}, \ldots$,

- fluent variables: $F, F^{\prime}, \ldots$,

- action variables: $A, A^{\prime}, \ldots$.

The initial situation is denoted by the constant $s_{0}$, and the two-ary function symbol $\operatorname{res}(A, S)$ denotes the situation that is reached when in situation $S$ the action $A$ has been performed. The relation symbol holds $(F, S)$ formalizes that the fluent $F$ is true in situation $S$.

For the YSP there are three actions (wait, load and shoot) and two fluents (alive and loaded). Initially a turkey called Fred is alive. We then load a gun, wait and shoot. The effect should be that Fred is dead after this sequence of actions. The common-sense argument from which this should follow is the

Law of Inertia: Things normally tend to stay the same.

Using our intuition from the last example, a natural formalization is given as follows:

$$
\begin{aligned}
& P_{Y S P}: \operatorname{holds}(F, \operatorname{res}(A, S)) \quad \leftarrow \operatorname{holds}(F, S), \operatorname{not} a b\left(r_{1}, A, F, S\right) \\
& \text { holds(loaded, res(load, } S)) \quad \leftarrow \\
& \left.a b\left(r_{1}, \text { shoot, alive, } S\right) \quad \leftarrow \text { holds(loaded, } S\right) \\
& \text { holds (alive, } \left.s_{0}\right)
\end{aligned}
$$

Such a straightforward formalization leads in most versions of classical nonmonotonic logic to the unexpected result, that Fred is not neccesarily dead. But obviously we expect to derive holds(alive, res $\left(\right.$ load, $\left.s_{0}\right)$ ) and

$$
\text { not holds(alive, res(shoot, res(wait, res } \left.\left.\left(\text { load, } s_{0}\right)\right)\right) \text { ) }
$$

Up to now we only have stated some very "natural" axiomatizations of given knowledge. We have motivated that something like default-negation " not" should be added to defi nite programs in order to do so and we have explicitly stated the answers to particular queries. What is still missing are solutions to the following very important problems

\footnotetext{
${ }^{5}$ To be formally correct we have to use many-sorted logic. But since all this could also be coded in predicate logic by using additional relation symbols, we do not emphasize this fact. We also understand that instantiations are done in such a way that the sorts are respected.
} 
- How should an appropriate query answering mechanism handling defaultnegation "not" look like?

- What is the formal semantics that such a procedural mechanism should be checked against?

Such a semantics is certainly not classical predicate logic because of the default character of "not" - not is not classical $\neg$. Both problems will be considered in detail in Section 3.

\subsection{What is a Semantics?}

In the last subsections we have introduced two principles (Orientation and Elimination of Tautologies) and used the term semantics of a program in a loose, imprecise way. We end this section with a precise notion of what we understand by a semantics.

As a first attempt, we can view a semantics as a mapping that associates to any program a set of positive atoms and a set of default atoms. In the case of SLD-Resolution the positive atoms are the ground instances of all derivable atoms. But sometimes we also want to derive default atoms (like in our two examples above). Our Orientation-Principle formalizes a minimal requirement for deriving such default-atoms.

Of course, we also want that a semantics SEM should respect the rules of $P$, i.e. whenever SEM makes the body of a rule true, then SEM should also make the head of the rule true. But it can (and will) happen that a semantics SEM does not always decide all atoms. Some atoms $A$ are not derivable nor are their defaultcounterparts not $A$. This means that a semantics SEM can view the body of a rule as being undefined.

This already happens in classical logic. Take the theory

$$
T:=\{(A \wedge B) \supset C, \neg A \supset B\} .
$$

What are the atoms and negated atoms derivable from $T$, i.e. true in all models of $T$ ? No positive atom nor any negated atom is derivable! The classical semantics therefore makes the truthvalue of $A \wedge B$ undefi ned in a sense.

Suppose a semantics SEM treats the body of a program rule as undefi ned. What should we conclude about the head of this rule? We will only require that this head is not treated as false by SEM-it could be true or undefi ned as well. This means that we require a semantics to be compatible with the program viewed as a 3-valued theory - the three values being "true", "false" and "undefined". For the understanding it is not neccessary to go deeper into 3 -valued logic. We simply note that we interpret " $\leftarrow$ " as the Kleene-connective which is true for "undefined $\leftarrow$ undefined" and false for "false $\leftarrow$ undefined".

Our discussion shows that we can view a semantics SEM as a 3-valued model of a program. In classical logic, there is a different viewpoint. For a given theory 
$T$ we consider there the set of all classical models $\operatorname{MOD}(T)$ as the semantics. The intersection of all these models is of course a 3-valued model of $T$, but $\operatorname{MOD}(T)$ contains more information. In order to formalize the notion of semantics as general as possible we defi ne

\section{Definition 2.4 (SEM).}

A semantics SEM is a mapping from the class of all programs into the powerset of the set of all 3-valued structures. SEM assigns to every program $P$ a set of 3-valued models of $P$ :

$$
\operatorname{SEM}(P) \subseteq \operatorname{MOD}_{3-v a l}^{\mathcal{L}_{P}}(P)
$$

This defi nition covers both the classical viewpoint (classical models are 2valued and therefore special 3-valued models) as well as our first attempt in the beginning of this subsection. Later on, in most cases we will be really interested only in Herbrand models.

Formally, we can associate to any semantics SEM in the sense of Defi nition 2.4 two entailment relations

sceptical: $\operatorname{SEM}^{\text {scept }}(P)$ is the set of all atoms or default atoms that are true in all models of $\operatorname{SEM}(P)$.

credulous: $\operatorname{SEM}^{\text {cred }}(P)$ is the set of all atoms or default atoms that are true in at least one model of $\operatorname{SEM}(P)$.

In this tutorial we only consider the sceptical viewpoint. Also, to facilitate notation, we will not formally distinguish between SEM and SEM ${ }^{s c e p t}$. In cases where by defi nition SEM can only contain a single model (like in the case of wellfounded semantics) we will omit the outer brackets and write

$$
\operatorname{SEM}(P)=M
$$

instead of $\operatorname{SEM}(P)=\{M\}$. We will also slightly abuse notation and write $l \in$ $\operatorname{SEM}(P)$ as an abbreviation for $l \in M$ for all $M \in \operatorname{SEM}(P)$.

\section{ADDING DEFAULT-NEGATION}

In the last section we have illustrated that logic programs with negation are very suitable for KR - they allow a natural and straightforward formalization of defaultstatements. The problem still remained to defi ne an appropriate semantics for this class and, if possible, to fi nd effi cient query-answering methods. Both points are adressed in this section.

We can distinguish between two quite different approaches:

LP-Approach: This is the approach taken mainly in the logic programming community. There one tried to stick as close as possible to SLD-resolution and 
treat negation as "fi nite-failure". This resulted in an extension of SLD, called SLDNF-resolution, a procedural mechanism for query answering. For a nice overview, we refer to [Apt and Bol, 1994].

NML-Approach: This is the approach suggested by non-monotonic reasoning people. Here the main question is "What is the right semantics?" I.e. we are looking fi rst for a semantics that correctly fi ts to our intuitions and treats the various KR-Tasks in the right (or appropriate) way. It should allow us to jump to conclusions even when only little information is available. Here it is of secondary interest how such a semantics can be implemented with a procedural calculus. Interesting overviews are [Minker, 1993; Minker, 1996] and [Dix, 1995c; Dix et al., 2001a].

The LP-approach is dealt with in Section 3.1. It is still very near to classical predicate logic-default negation is interpreted as fi nite-failure. To get a stronger semantics, we interpret "not" as failure in Section 3.2. The main difference is that the principle Elimination of Tautologies holds. We then introduce a principle GPPE which is related to partial evaluation. In KR one can see this principle as allowing for defi nitional extensions-names or abbreviations can be introduced without changing the semantics.

All these principles do not yet determine a unique semantics - there is still room for different semantics and a lot of them have been defi ned in the last years. We do not want to present the whole zoo of semantics nor to discuss their merits or shortcomings. We refer the reader to the overview articles [Apt and Bol, 1994] and [Dix, 1995c] and the references given therein. We focus on the two main competing approaches that still have survived. These are the wellfounded semantics WFS (Section 3.3) and the stable semantics STABLE (Section 3.4). Finally, in Section 3.5 we discuss complexity and expressibility results for the semantics presented so far.

\subsection{Negation-as-Finite-Failure}

The idea of negation treated as fi nite-failure can be best illustrated by still considering defi nite programs, but queries containing default-atoms. How should we handle such default-atoms by modifying our SLD-resolution? Let us try this:

- If we reach a default-atom "not $A$ " as a subgoal of our original query, we keep the current SLD-tree in mind and start a new SLD-tree by trying to solve " $A$ ".

- If this succeeds, then we falsifi ed "not $A$ ", the current branch is failing and we have to backtrack and consider a different subquery.

- But it can also happen that the SLD-tree for " $A$ " is fi nite with only failing branches. Then we say that $A$ fi nitely fails, we turn back to our original 
SLD-tree, consider the subgoal "not $A$ " as successfully solved and go on with the next subgoal in the current list.

It is important to note that an SLD-tree for a positive atom can fail without being fi nite. The SLD-tree for the program consisting of the single rule $p \leftarrow p$ with respect to the query $p$ is infi nite but failing (it consists of one single infi nite branch). In Figure 1 on page 8 the leftmost branch is also failing but infi nite.

Although this idea of fi nite-failure is very procedural in nature, there is a nice model theoretical counterpart-Clark's completion $\operatorname{comp}(P)$ ([Clark, 1978]). The idea of Clark was that a program $P$ consists not only of the implications, but also of the information that these are the only ones. Roughly speaking, he argues that one should interpret the " $\leftarrow$ "-arrows in rules as equivalences " $\equiv$ " in classical logic. We do not give the exact defi nitions here, as they are very complex; in the nonpropositional case, a symbol for equality, together with axioms describing it $^{6}$, has to be introduced. However, for the propositional case, $\operatorname{comp}(P)$ is obtained from $P$ by just

1. collecting all given clauses with the same head into one new "clause" with this respective head and a disjunctive body (containing all bodies of the old clauses), and

2. replacing the implication-symbols “ $\leftarrow$ ” by “三”.

Definition 3.1 (Clark's Completion $\operatorname{comp}(P)$ ).

Clark's semantics for a program $\mathrm{P}$ is given by the set of all classical models of the theory $\operatorname{comp}(P)$.

We can now see the classical theory $\operatorname{comp}(P)$ as the information contained in the program $P$. $\operatorname{comp}(P)$ is like a sort of closed world assumption applied to $P$. We are now able to derive negative information from $P$ by deriving it from $\operatorname{comp}(P)$. In fact, the following soundness and completeness result for defi nite programs $P$ and defi nite queries $Q=\bigwedge_{i} A_{i}$ (consisting of only positive atoms) holds:

\section{Theorem 3.1 (COMP and Fair FF-Trees).}

The following conditions are equivalent:

- $\operatorname{comp}(P) \models \forall \neg Q$

- Every fair SLD-tree for $P$ with respect to $Q$ is fi nitely failed.

Note that in the last theorem we did not use default negation but classical negation $\neg$ because we just mapped all formulae into classical logic. We need the fairness assumption to ensure that the selection of atoms is reasonably well-behaving: we want that every atom or default-atom occurring in the list of preliminary goals will eventually be selected.

\footnotetext{
${ }^{6}$ CET: Clark's Equational Theory. $\operatorname{CET}\left(\mathcal{L}_{P}\right)$ axiomatizes the equality theory of all $\operatorname{Herbrand}\left(\mathcal{L}_{P}\right)$ models. See [Mancarella et al., 1988; Shepherdson, 1992] for the problem of equality and the underlying language.
} 
But even this result is still very weak-after all we want to handle not only negative queries but programs containing default-atoms. From now on we consider programs with default-atoms in the body. We usually denote them by

$$
A \leftarrow \mathcal{B}^{+} \wedge \operatorname{not} \mathcal{B}^{-}
$$

where $\mathcal{B}^{+}$contains all the positive body atoms and not $\mathcal{B}^{-}$all default atoms "not $C$ ".

Our two motivating examples in Section 2.4 contain such default atoms. This gives rise to an extension of SLD, called SLDNF, which treats negation as FiniteFailure

$$
\mathrm{SLDNF}=\mathrm{SLD}+\operatorname{not} L \text { succeeds, if } L \text { fi nitely fails. }
$$

The precise defi nitions of SLDNF-resolution, tree, etc. are very complex: we refer to [Lloyd, 1987; Apt, 1990]. Apt and Bol gave interesting improved versions of these notions: see [Apt and Bol, 1994, Section 3.2]. In order to get an intuitive idea, it is suffi cient to describe the following underlying principle:

\section{Principle 3 (A “Naive” SLDNF-Resolution).}

If in the construction of an SLDNF-tree a default-atom not $L_{i j}$ is selected in the list $\mathcal{L}_{i}=\left\{L_{i 1}, L_{i 2}, \ldots\right\}$, then we try to prove $L_{i j}$.

If this fails fi nitely (it fails because the generated subtree is fi nite and failing), then we take not $L_{i j}$ as proved and we go on to prove $L_{i(j+1)}$.

If $L_{i j}$ succeeds, then not $L_{i j}$ fails and we have to backtrack to the list $\mathcal{L}_{i-1}$ of preliminary subgoals (the next rule is applied: "backtracking").

Does SLDNF-resolution properly handle Examples 2.4 on page 12 and 2.5 on page 13? It does indeed:

Inheritance: The query make_top (Tweety) generates an SLD-tree with one main branch, the nodes of which are:

$$
\begin{aligned}
& \text { flies }(\text { Tweety }), \\
& \operatorname{bird}(\text { Tweety }), \operatorname{not} a b\left(r_{1}, \text { Tweety }\right) \text {, } \\
& \text { not } a b\left(r_{1}, \text { Tweety }\right), \\
& \text { Success. }
\end{aligned}
$$

The third node has a sibling-node penguin(Tweety), not $a b\left(r_{1}\right.$, Tweety) which immediately fails because Tweety does not unify with Sam. The Success-node is obtained from not $a b\left(r_{1}\right.$, Tweety) because the corresponding SLD-tree for $a b\left(r_{1}\right.$, Tweety) fails fi nitely (this tree consists only of $a b\left(r_{1}\right.$, Tweety) and penguin(Tweety $\left.)\right)$.

YSP: The crucial query is

$$
\text { ?- not holds(alive, res(shoot, res(wait, res } \left.\left.\left(\text { load, } s_{0}\right)\right)\right) \text { ). }
$$

So we consider?-holds(alive, res(shoot, res(wait, res $\left(\right.$ load, $\left.\left.\left.s_{0}\right)\right)\right)$ ). Again the SLD-tree for this query consists mainly of one branch: the nodes are 


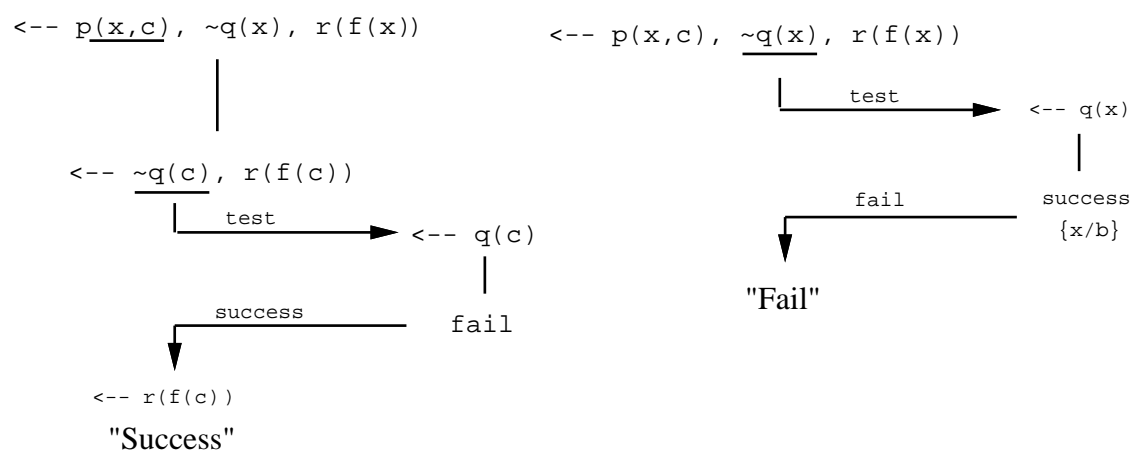

Figure 2. The Floundering-Problem

obtained from the query by applying successively the fi rst program rule (law of inertia). By evaluation of the holds-predicate, we eventually arrive at the fact holds(alive, $\left.s_{0}\right)$ and the "not $a b$ " predicates remain to be solved. For any of these predicates we again have to consider separate SLDtrees. But for $a b\left(r_{1}\right.$, shoot, alive, res (wait, res $\left(\right.$ load, $\left.\left.\left.s_{0}\right)\right)\right)$ it is easy to see that the associated tree already fi nitely fails (because it generates the subgoal " not $a b\left(r_{1}\right.$, wait, loaded, res $\left(\right.$ load, $\left.\left.s_{0}\right)\right)$ " the corresponding SLD-tree of which immediately fi nitely fails) and therefore, since no backtracking is possible, the tree for

$$
\text { ?- holds(alive, res(shoot, res(wait, res } \left.\left.\left(\text { load, } s_{0}\right)\right)\right) \text { ) }
$$

fi nitely fails and our original query succeeds: Fred is dead.

Up to now it seems that SLDNF-resolution solves all our problems. It handles our examples correctly, and is defi ned by a procedural calculus strongly related to SLD. There are two main problems with SLDNF:

- SLDNF can not handle free variables in negative subgoals,

- SLDNF is still too weak for knowledge representation.

The latter problem is the most important one. By looking at a particular example, we will motivate in Section 3.2 the need for a stronger semantics. This will lead us in the remaining sections to the wellfounded and the stable semantics.

For the rest of this section we consider the fi rst problem, known as the floundering problem. This problem will also occur later in implementations of the wellfounded or the stable semantics. We consider the program $P_{\text {flounder }}$ consisting of the three facts

$$
p(c, c), q(b), r(f(c)) .
$$


Our query is ? $-p(x, c)$, not $q(x), r(f(x))$, that is, we are interested in instantiations of $x$ such that the query follows from the program. The situation is illustrated in Figure 2 on the page before. Let us suppose that we always select the first atom or default-atom: it is underlined in the sequel. The SLDNF-tree of this trivial example is linear and has three nodes: the fi rst node is the query itself

$$
?-\underline{p(x, c)}, \operatorname{not} q(x), r(f(x)),
$$

the second node is ? - not $q(c), r(f(c))$. Now, we enter the negation-as-failure mode and ask $?-q(c)$. This query immediately fails (the generated tree exists, is fi nite and fails) so that we give back the answer "yes, the default atom not $q(c)$ succeeds and can be skipped from the list". The last node is ? $-\underline{r(f(c)) \text { which }}$ immediately succeeds.

Note that in the last step, the test for $?-q(c)$ has to be fi nished before the tree can be extended. If we get no answer, the SLDNF-tree simply does not exist: this can not happen with SLD-trees.

So far everything was fi ne. But what happens if we select the second atom in the fir rst step

$$
?-p(x, c), \underline{\operatorname{not} q(x)}, r(f(x)) ?
$$

\section{Example 3.1 (Floundering).}

We again consider the program $P_{\text {flounder }}$ consisting of the three facts

$$
p(c, c), q(b), r(f(c)) .
$$

Our query is ? $-p(x, c)$, not $q(x), r(f(x))$, and in the fi rst step we will select the second default-atom, i.e. one with a free variable. Thus we enter the negation-asfailure mode with the query ? - not $q(x)$. In this case, $x$ may be instantiated to $b$ so that we have to give back the answer "no, the default-atom not $q(x)$ fails" and the whole query will fail. This is because SLDNF treats the subgoal as " $\forall x$ not $q(x)$ " instead of " $\exists x$ not $q(x)$ " which is intended. There exist approaches to overcome this shortcoming by treating negation as constructive negation: see [Chan, 1988; Chan and Wallace, 1989; Drabent, 1994].

In the classical SLDNF-resolution negation-as-fi nite-failure is only a test, no bindings are produced. On the one hand this may be considered a shortcoming, on the other hand, it makes the SLDNF procedure more tractable. Note that the problem to decide if a given program flounders is undecidable [Börger, 1987]). See also [Shepherdson, 1991] for more unsolvable problems related to SLDNF.

SLDNF is a procedural mechanism. It would be nice to have a modeltheoretical counterpart. In Theorem 3.1 on page 17 we already related a restricted form of fi nite failure to Clark's completion. We will see later that $\operatorname{comp}(P)$ is inconsistent even in cases where we would not expect it. Therefore Fitting [Fitting, 1985] introduced a three-valued formulation $\operatorname{comp}_{3}(P)$ of the original completion. Kunen ([Kunen, 1987]) then proved in the propositional case SLDNF is sound and complete with respect to $\mathrm{comp}_{3}(P)$. 
In the predicate logic case, SLDNF is not complete but it is always correct [Shepherdson, 1988, Theorem 39]) with respect to $c o m p_{3}(P)$ : given a query $Q$,

- if SLDNF succeeds with answer $\Theta$, then $c o m p_{3}(P) \models_{3} \forall Q \Theta$, and

- if SLDNF fails, then $\operatorname{comp}_{3}(P) \models_{3} \neg \exists Q$.

This correctness result is also the reason for the incompleteness of SLDNF with respect to two-valued $\operatorname{comp}(P)$. It states that any formula derivable by SLDNF is a three-valued consequence of $c o m p_{3}(P)$. But, since there are two-valued consequences of a theory that are not three-valued ones (three-valued logic is weaker than two-valued logic), SLDNF can not be complete. Extensions of the above completeness result to certain subclasses of predicate logic programs require severe restrictions on the syntactic form of $P$. To defi ne these syntactic restrictions, we need the notion of the dependency-graph:

\section{Definition 3.2 (Dependency-Graph $\mathcal{G}_{P}$ ).}

For a logic program $P$ with negation, the dependency graph $\mathcal{G}_{P}$ is a fi nite directed graph whose vertices are the predicate symbols from $P$. There is a positive (respectively negative) edge from $R$ to $R^{\prime}$ iff there is a clause in $\mathrm{P}$ with $R$ in its head and $R^{\prime}$ occurring positively (respectively negative) in its body.

We also say

- $R$ depends on $R^{\prime}$ if there is a path in $\mathcal{G}_{P}$ from $R$ to $R^{\prime}$ (by defi nition, $R$ depends on itself),

- $R$ depends positively (resp. negatively) on $R^{\prime}$ if there is a path in $\mathcal{G}_{P}$ from $R$ to $R^{\prime}$ containing only positive edges (resp. at least one negative edge). (by defi nition $R$ depends positively on itself),

- $R$ depends evenly (resp. oddly) on $R^{\prime}$ if there is a path in $\mathcal{G}_{P}$ from $R$ to $R^{\prime}$ containing an even (resp. odd) number of negative edges (by defi nition $R$ depends evenly on itself).

The following properties of a program $P$ turn out to be very important:

stratifi ed: no predicate depends negatively on itself,

strict: there are no dependencies that are both even and odd,

call-consistent: no predicate depends oddly on itself ${ }^{8}$,

allowedness: every variable occurring in a clause must occur in at least one positive atom of the body of that clause.

Strictness and allowedness turn out to be the most important restrictions that imply completeness results for SLDNF:

\footnotetext{
${ }^{7}$ or: there are no cycles containing at least one negative edge.

${ }^{8}$ or: there are no odd cycles.
} 


\begin{tabular}{||c|c|l||}
\hline \hline Prog. $\mathbf{P}$ & Semantics & Completeness \\
\hline \hline $\begin{array}{c}\text { allowed + hierarchical } \\
\text { allowed + stratifi ed } \\
\text { allowed }\end{array}$ & $\begin{array}{c}\operatorname{comp}(P) \\
\operatorname{comp}(P) \\
\operatorname{comp}_{3}(P)\end{array}$ & $\begin{array}{l}\text { yes, no recursion at all } \\
\text { yes, if } P \cup\{\leftarrow A\} \text { strict } \\
\text { yllowed + call-consistent } \vdash_{3}\end{array}$ \\
\hline $\operatorname{comp}(P)$ & $\begin{array}{l}\text { yes, if } P \cup\{\leftarrow A\} \text { strict: } \\
\operatorname{comp}(P) \vdash \forall A \text { iff } \\
\operatorname{comp} p_{3}(P) \vdash_{3} \forall A\end{array}$ \\
\hline \hline
\end{tabular}

Table 1. Completeness for SLDNF

While strictness excludes situations of the form $p(x) \leftarrow q(x), p(c) \leftarrow \neg q\left(f\left(c^{\prime}\right)\right)$, allowedness excludes constructs of the form equal $(x, x) \leftarrow$ and also solves the floundering-problem.

Strictness implies that $\operatorname{comp}_{3}(P)$ and $\operatorname{comp}(P)$ are equivalent [Kunen, 1989])

$$
\operatorname{comp}_{3}(P) \models_{3} \forall Q \Theta \text { iff } \operatorname{comp}(P) \models \forall Q \Theta .
$$

Table 1 gives an overview of the different completeness results. Note that the query $A$ is always considered to be allowed.

Much work was done in LP (see [Decker and Cavedon, 1990; Barbuti and Martelli, 1986; Stärk, 1994]) to fi nd other syntactically characterizable classes, for which SLDNF is also complete.

\subsection{Negation-as-Failure}

Let us first illustrate that SLDNF answers quite easily our requirements of a semantics SEM (stated explicitly in Defi nition 2.4 on page 15 ). We can formulate these requirements as two program-transformations (they will be used later for computing a semantics). We call them reductions for obvious reasons.

\section{Principle 4 (Reduction).}

Suppose we are given a program $P$ with possibly default-atoms in its body. If a ground atom $A$ does not unify with any head of the rules of $P$, then we can delete in every rule any occurrence of "not $A$ " without changing the semantics.

Dually, if there is an instance of a rule of the form " $B \leftarrow$ " then we can delete all rules that contain " not $B$ " in their bodies.

It is obvious that SLDNF "implements" these two reductions automatically. The weakness of SLDNF for knowledge representation is in a sense inherited from SLD. When we consider rules of the form " $p \leftarrow p$ ", then SLD resolution gets 
into an infi nite loop and no answer to the query ?- $p$ can be obtained. This has often the effect that when we enter into negation-as-failure mode, the SLD-tree to be constructed is not fi nite, although it is not successful and therefore should be considered as failed.

Let us discuss this point with a more serious example.

\section{Example 3.2 (The Transitive Closure).}

Assume we are given a graph consisting of nodes and edges between some of them. We want to know which nodes are reachable from a given one. A natural formalization of the property "reachable" would be

$$
\text { reachable }(x) \leftarrow \text { edge }(x, y) \text {, reachable }(y) .
$$

What happens if we are given the following facts

$$
\operatorname{edge}(a, b), \operatorname{edge}(b, a), \operatorname{edge}(c, d)
$$

and reachable $(c)$ ? Of course, we expect that neither $a$ nor $b$ are reachable because there is no path from $c$ to either $a$ or $b$.

But SLDNF-Resolution does not derive "not reachable (a)"!

How does this result relate to Theorem 3.1 on page 17 ? Note that our query has exactly the form as required there. Clark's completion of our program rule is

$$
\text { reachable }(x) \equiv(x \doteq c \vee \exists y(\text { reachable }(y) \wedge \operatorname{edge}(y, x)))
$$

from which, together with our facts about the edge-relation, $\neg$ reachable $(a)$ is indeed not derivable. This is due to the wellknown fact that transitive closure is not expressible in fi rst order predicate logic.

Note also that our Principle 2 on page 10 does not help, because it simply does not apply. It turns out that we can augment our two principles by a third one, that constitutes together with them a very nice calculus handling the above example in the right way. This principle is related to partial evaluation, hence its name GPPE ${ }^{9}$. Let us motivate this principle with the last example. The query "not reachable $(a)$ " leads to "reachable $(a) \leftarrow$ edge $(a, b)$, reachable $(b)$ " and "reachable $(b)$ " leads to "reachable $(b) \leftarrow$ edge $(b, a)$, reachable $(a)$ ". Both rules can be seen as defi nitions for reachable $(a)$ and reachable $(b)$ respectively. So it should be possible to replace in these rules the body atoms of reachable by their defi nitions. Thus we obtain the two rules

$$
\begin{aligned}
& \text { reachable }(a) \leftarrow \operatorname{edge}(a, b), \text { edge }(b, a), \text { reachable }(a) \\
& \text { reachable }(b) \leftarrow \operatorname{edge}(b, a), \text { edge }(a, b), \text { reachable }(b)
\end{aligned}
$$

that can both be eliminated by applying Principle 2 on page 10. So we end up with a program that does neither contain reachable $(a)$ nor reachable $(b)$ in one

\footnotetext{
${ }^{9}$ Generalized Principle of Partial Evaluation
} 
of the heads. Therefore, according to Principle 1 on page 7 both atoms should be considered false. The precise formulation of this principle is as follows:

Principle 5 (GPPE, [Brass and Dix, 1994; Sakama and Seki, 1994]).

We say that a semantics SEM satisfi es GPPE, if the following transformation does not change the semantics. Replace a rule $A \leftarrow \mathcal{B}^{+} \wedge$ not $\mathcal{B}^{-}$where $\mathcal{B}^{+}$contains a distinguished atom $B$ by the rules

$$
A \leftarrow\left(\mathcal{B}^{+} \backslash\{B\}\right) \cup \mathcal{B}_{i}^{+} \wedge \operatorname{not}\left(\mathcal{B}^{-} \cup \mathcal{B}_{i}^{-}\right)(i=1, \ldots, n)
$$

where $B \leftarrow \mathcal{B}_{i}^{+} \wedge \operatorname{not} \mathcal{B}_{i}^{-}(i=1, \ldots, n)$ are all rules with head $B$.

Note that any semantics SEM satsfying GPPE and Elimination of Tautologies can be seen as extending SLD by doing some Loop-checking. We will call such semantics NMR-semantics in order to distinguish them from the classical $L P$ semantics which are based on SLDNF or variants of Clark's completion $\operatorname{comp}(P)$ :

- NMR-Semantics $=$ SLDNF + Loop-check .

The following, somewhat artifi cial example illustrates this point.

\section{Example 3.3 (COMP vs. NMR).}

$$
\begin{aligned}
& P_{N M R}: p \leftarrow p \\
& q \leftarrow \operatorname{not} p \\
& P_{N M R}^{\prime}: p \leftarrow p \\
& q \leftarrow \operatorname{not} p \\
& r \leftarrow \operatorname{not} r \\
& \begin{aligned}
\operatorname{comp}\left(P_{N M R}\right): \quad p & \equiv p \\
q & \equiv \neg p
\end{aligned} \\
& \operatorname{comp}\left(P_{N M R}^{\prime}\right): p \equiv p \\
& \begin{aligned}
q & \equiv \neg p \\
r & \equiv \neg r
\end{aligned}
\end{aligned}
$$

For both programs, the answers of the completion-semantics do not match our NMR-intuition! In the case of $P_{N M R}$ we expect $q$ to be derivable, since we expect not $p$ to be derivable: the only possibility to derive $p$ is the rule $p \leftarrow p$ which, obviously, will never succeed. But $q \notin T h(\{q \equiv \neg p\})=\operatorname{comp}\left(P_{N M R}\right)$ ! In the case of $P_{N M R}^{\prime}$ we expect $p$ not to be derivable, for the same reason: the only possibility to derive $p$ is the rule $p \leftarrow p$. But $p \in F m l=T h(\{r \equiv \neg r\})=$ $\operatorname{comp}\left(P_{N M R}^{\prime}\right)$ !

Note that the answers of the completion-semantics agree with the mechanism of SLDNF: $p \leftarrow p$ represents a loop. The completion of $P^{\prime}$ is inconsistent: this led Fitting to consider the three-valued version of $\operatorname{comp}(P)$ mentioned at the end of Section 3.1. This approach avoids the inconsistency (the query ? $-p$ is not answered "yes") but it still does not answer "no" as we would like to have. 
The last principle in this section is related to subsumption: we can get rid of non-minimal rules by simply deleting them.

Principle 6 (Subsumption).

In a program $P$ we can delete a rule $A \leftarrow \mathcal{B}^{+} \wedge$ not $\mathcal{B}^{-}$whenever there is another rule $A \leftarrow \mathcal{B}^{\prime+} \wedge \operatorname{not} \mathcal{B}^{\prime-}$ with

$$
\mathcal{B}^{+} \subseteq \mathcal{B}^{+} \text {and } \mathcal{B}^{\prime-} \subseteq \mathcal{B}^{-} .
$$

As a simple example, the rule $A \leftarrow B, C$, not $D$, not $E$ is subsumed by the 3 rules $A \leftarrow C, \operatorname{not} D, \operatorname{not} E$ or $A \leftarrow B, C, \operatorname{not} E$ and by $A \leftarrow C$, not $E$.

\subsection{The Wellfounded Semantics: WFS}

The wellfounded semantics, originally introduced in [Van Gelder et al., 1988], is the weakest semantics satisfying our 4 principles (see [Brass and Dix, 1999; Brass and Dix, 1998; Dix, 1995b]). We call a semantics

$$
\mathrm{SEM}_{1} \text { weaker than } \mathrm{SEM}_{2} \text {, written } \mathrm{SEM}_{1} \leq_{k} \mathrm{SEM}_{2} \text {, }
$$

if for all programs $P$ and all atoms or default-atoms $l$ the following holds:

$$
\operatorname{SEM}_{1}(P) \models l \text { implies } \operatorname{SEM}_{2}(P) \models l .
$$

I.e. all atoms derivable from $\mathrm{SEM}_{1}$ with respect to $P$ are also derivable from $\mathrm{SEM}_{2}$. The notion $\leq_{k}$ refers to the knowledge ordering in three-valued logic. This is a nice theorem and gives rise to the following defi nition:

Theorem 3.2 (WFS, [Brass and Dix, 1999]).

There exists the weakest semantics satisfying our four principles Elimination of Tautologies, Reduction, Subsumption and GPPE. This semantics is called wellfounded semantics WFS.

It can also be shown, that for propositional programs, our transformations can be applied to compute this semantics.

\section{Theorem 3.3 (Confluent Calculus for WFS,[Brass and Dix, 1998]).}

The calculus consisting of these four transformations is confluent, i.e. whenever we arrive at an irreducible program, it is uniquely determined. The order of the transformations does not matter.

For fi nite propositional programs, it is also terminating: any program $P$ is therefore associated a unique normalform res $(P)$. The wellfounded semantics of $P$ can be read off from $\operatorname{res}(P)$ as follows

$$
W F S(P)=\{A: A \leftarrow \in \operatorname{res}(P)\} \cup\{\operatorname{not} A: A \text { is in no head of } \operatorname{res}(P)\}
$$

We note that the size of the residual program is in general exponential in the size of the original program. Recently it was shown in [Brass et al., 2001b] how a 
small modifi cation of the residual program, which still satisfi es the nice characterization of computing WFS as given in Theorem 3.3 on the page before, results in a polynomial computation.

Therefore the wellfounded semantics associates to every program $P$ with negation a set consisting of atoms and default-atoms. This set is a 3-valued model of $P$. It can happen, of course, that this set is empty. But it is always consistent, i.e. it does not contain an atom $A$ and its negation not $A$. Moreover, it extends SLDNF: whenever SLDNF derives an atom or default-atom and does not flounder, then WFS derives it as well. Therefore the two examples of Section 2.4 are handled in the right way. But also for Example 3.2 on page 23 we get the desired answers.

Let us discuss whether every sequence of program transformations terminates, i.e. if our calculus is strongly terminating. Already the simple program consisting of just the loop " $A \leftarrow A$ " shows a problem. Applying GPPE leads to the same program, so GPPE can be applied infi nitely often without leading to the residual program. Of course, in this particular case an application of the Elimination of Tautologies immediately leads to the residual program. But still another problem can occur ${ }^{10}$ :

$$
\begin{aligned}
P_{\text {loop }}: A & \leftarrow B \\
B & \leftarrow A \\
C & \leftarrow A, \neg C
\end{aligned}
$$

If we apply $\mathrm{GPPE}_{A}$ to the third clause, this clause is replaced by $C \leftarrow B, \neg C$. We can now apply $\mathrm{GPPE}_{B}$ again to this clause and get the original program. So we have an oscillation

$$
G P P E_{B} \circ G P P E_{A}\left(P_{l o o p}\right)=P_{l o o p}
$$

To summarize, not every sequence of transformations terminates. But a simple additional property will ensure this.

\section{Definition 3.3 (Fair Sequences).}

We call a sequence of program transformations fair, if in the corresponding sequence of programs

1. every positive body-atom is eventually removed (either by removing the whole clause using a suitable transformation or by an application of GPPE), and

2. every tautology clause is eventually removed (either by applying Elimination of Tautologies or another suitable transformation).

\section{Theorem 3.4 (Strong Termination for Fair Sequences).}

Our calculus of transformations is strongly terminating for fair sequences of transformations. Such fair sequences therefore always lead to the residual program.

\footnotetext{
${ }^{10}$ brought to our attention by Frieder Stolzenburg
} 
As we said above, loop-checking is in general undecidable. Therefore WFS is in the most general case where variables and function-symbols are allowed, undecidable. Only for fi nite propositional programs it is decidable. In fact, it is of quadratic complexity (see Section 3.5).

Let us end this section with another example, which contains negation.

\section{Example 3.4 (Van Gelder's Example).}

Assume we are describing a two-players game like checkers. The two players alternately move a stone on a board. The moving player wins when his opponent has no more move to make. We can formalize that by

- $\operatorname{wins}(x) \leftarrow$ move_from_to $_{-}(x, y)$, not wins $(y)$

meaning that

- the situation $\mathrm{x}$ is won (for the moving player A), if he can lead over ${ }^{11}$ to a situation $y$ that can never be won for B.

Assume we also have the facts

move_from_to $(a, b), m_{-} e_{-} f r o m_{-} t o(b, a)$ and move_from_to $(b, c)$.

Our query to this program $P_{\text {game }}$ is ?- wins $(b)$. Here we have no problems with floundering, but using SLDNF we get an infi nite sequence of oscillating SLD-trees (none of which fi nitely fails).

WFS, however, derives the right results

$$
W F S\left(P_{\text {game }}\right)=\{\text { not wins }(c), \text { wins }(b), \text { not wins }(a)\}
$$

which matches completely with our intuitions.

\subsection{The Stable Semantics: STABLE}

We defi ned WFS as the weakest semantics satisfying our four principles. This already indicates that there are even stronger semantics. One of the main competing approaches is the stable semantics STABLE. The stable semantics associates to any program $P$ a set of 2 -valued models, like classical predicate logic. STABLE satisfi es the following property, in addition to those that have been already introduced:

\section{Principle 7 (Elimination of Contradictions).}

Suppose a program $P$ has a rule which contains the same atom $A$ and not $A$ in its body. Then we can eliminate this rule without changing the semantics.

This principle can be used, in conjunction with the others to defi ne the stable semantics

\footnotetext{
${ }^{11}$ With the help of a regular move, given by the relation move $_{-}$from_to $/ 2$.
} 
Theorem 3.5 (STABLE, [Brass and Dix, 1997]).

There exists the weakest semantics satisfying our fi ve principles Elimination of Tautologies, Reduction, Subsumption, GPPE and Elimination of Contradictions.

If a semantics SEM satisfi es Elimination of Contradictions it is based on 2valued models ([Brass and Dix, 1997]). The underlying idea of STABLE is that any atom in an intended model should have a defi nite reason to be true or false. This idea was made explicit in [Bidoit and Froidevaux, 1991a; Bidoit and Froidevaux, 1991b] and, independently, in [Gelfond and Lifschitz, 1988]. We use the latter terminology and introduce the Gelfond-Lifschitz transformation: for a program $P$ and a model $N \subseteq B_{P}$ we defi ne

$$
P^{N}:=\left\{\text { rule }^{N}: \text { rule } \in P\right\}
$$

where rule $:=A \leftarrow B_{1}, \ldots, B_{n}$, not $C_{1}, \ldots, \operatorname{not} C_{m}$ is transformed as follows

$$
(\text { rule })^{N}:= \begin{cases}A \leftarrow B_{1}, \ldots, B_{n}, & \text { if } \forall j: C_{j} \notin N, \\ \mathbf{t}, & \text { otherwise. }\end{cases}
$$

Note that $P^{N}$ is always a defi nite program. We can therefore compute its least Herbrand model $M_{P^{N}}$ and check whether it coincides with the model $N$ with which we started:

Definition 3.4 (STABLE).

$N$ is called a stable model ${ }^{12}$ of $P$ iff $M_{P^{N}}=N$.

What is the relationship between STABLE and WFS? We have seen that they are based on rather identical principles.

- Stable models $N$ extend WFS: $l \in \mathrm{WFS}(P)$ implies $N=l$.

- If $\operatorname{WFS}(P)$ is two-valued, then $\operatorname{WFS}(P)$ is the unique stable model.

But there are also differences. We refer to Example 3.4 on the page before and consider the program $P$ consisting of the clause

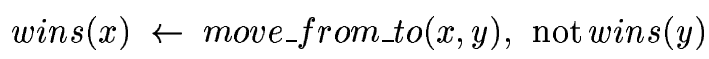

together with the following facts: move $f r o m_{-} t o(a, b)$, move_from_to $_{-}(b, a)$, as well as move_from_to $(b, c)$, and move $f r o m_{-} t o(c, d)$. In this particular case we have two stable models: $\{$ wins $(a)$, wins $(c)\}$ and $\{\operatorname{wins}(b)$, wins $(c)\}$ and therefore

$$
\operatorname{WFS}(P)=\{\operatorname{wins}(c), \text { not wins }(d)\}=\bigcap_{\mathcal{N} \text { a stable model of } P} \mathcal{N} .
$$

This means that the 3-valued wellfounded model is exactly the set of all atoms or default-atoms true in all stable models. But this is not always the case, as the program of $P_{\text {splitting }}$ shows:

\footnotetext{
${ }^{12}$ Note that we only consider Herbrand models.
} 
Example 3.5 (Reasoning by cases).

$$
\begin{aligned}
P_{\text {splitting }}: a & \leftarrow \operatorname{not} b \\
b & \leftarrow \operatorname{not} a \\
p & \leftarrow a \\
p & \leftarrow b
\end{aligned}
$$

Although neither $a$, nor $b$ can be derived in any semantics based on two-valued models (as STABLE for example), the disjunction $a \vee b$, thus also $p$, is true. In this way the example is handled by the completion semantics, too. WFS $(P)$, however, is empty; if the WFS cannot decide between $a$ or not $a$, then $a$ is undefi ned.

The main differences between STABLE and WFS are

- STABLE is not always consistent,

- STABLE does not allow for a goal-oriented implementation.

The inconsistency comes from odd, negative cycles

$$
S T A B L E(p \leftarrow \operatorname{not} p)=\emptyset .
$$

The idea to consider 2-valued models for a semantics neccessarily implies its inconsistency ([Brass and Dix, 1997]). Note that $W F S(p \leftarrow \operatorname{not} p)=\{\emptyset\}$ which is quite different! Suffi cient criteria for the existence of stable models are contained in [Dung, 1992; Fages, 1993].

That STABLE does not allow for a Top-Down evaluation is a more serious drawback and has nothing to do with inconsistency. This behaviour led Dix to defi ne the notion of Relevance and Modularity (see Section 7.1 and [Dix, 1992a; Dix, 1992b; Dix, 1995b]. Bry reinvented Modularity (he termed it compositionality) and argued that a semantics should satisfy it.

\section{Example 3.6 (STABLE is not Goal-Oriented).}

$$
\begin{aligned}
& P_{\text {rel }(a)}: a \leftarrow \operatorname{not} b \quad P: a \leftarrow \operatorname{not} b \\
& b \leftarrow \operatorname{not} a \quad b \leftarrow \operatorname{not} a \\
& p \leftarrow \operatorname{not} p \\
& p \leftarrow a
\end{aligned}
$$

$P_{r e l(a)}$ is the subprogram of $P$ that consists of all rules that are relevant to answer the query ?- $a$. It has two stable models $\{a\}$ and $\{b\}-a$ is not true in all of them. But the program $P$ has the unique stable model $\{p, a\}$, so $a$ is true in all stable models of $P$.

The last example shows that the truthvalue of an atom $a$ also depends on atoms that are totally unrelated with $a$ ! This is considered a drawback of STABLE by many people. Note that a straightforward modifi cation of STABLE is not possible ([Dix and Müller, 1994b; Dix and Müller, 1994c]). 
We end this section with another description of WFS and STABLE that will be useful in later sections. It was introduced in [Baral and Subrahmanian, 1991; Baral and Subrahmanian, 1992]:

Definition 3.5 (Antimonotone Operator $\gamma_{P}$ ).

For a program $P$ and a set $N \subset B_{P}$ we defi ne an operator $\gamma_{P}$ mapping Herbrandstructures to Herbrand structures:

$$
\gamma_{P}(N):=M_{P^{N}}
$$

It is easy to see that $\gamma_{P}$ is antimonotone. Therefore its twofold application $\gamma^{2}$ is monotone ([Tarski, 1955]).

Obviously, the stable models of a program $P$ are exactly the fi xpoints of $\gamma_{P}$. This is just a reformulation of Defi nition 3.4 on page 28. WFS is related to $\gamma$ as follows

Theorem 3.6 (WFS and $\gamma^{2}$ ).

A positive atom $A$ is in WFS(P) iff $A \in l f p\left(\gamma_{P}^{2}\right)$. A default-atom $\operatorname{not} A$ is in WFS(P) iff $A \notin g f p\left(\gamma_{P}^{2}\right)$ :

$$
W F S(P)=l f p\left(\gamma_{P}^{2}\right) \cup\left\{\operatorname{not} A: A \notin g f p\left(\gamma_{P}^{2}\right)\right\} .
$$

Atom or default-atoms that do occur in neither of the two sets are undefi ned.

\subsection{Complexity and Expressibility}

In this section we collect some complexity results for the semantics considered so far. The reason why NMR-semantics are in the general case (free variables and function symbols) undecidable is strongly related to loop-checking. Let us consider the program

$$
P(x) \leftarrow P(f(x))
$$

or, equivalently, the infi nite propositional program

$$
p_{0} \leftarrow p_{1}, p_{1} \leftarrow p_{2}, \ldots, p_{i} \leftarrow p_{i+1}, \ldots
$$

Any NMR-semantics should derive " not $P(t)$ " (resp. " not $p_{i}$ ") for all terms $t$, but a procedure to detect such infi nite loops is impossible in general. Our principles GPPE and Elimination of Tautologies can detect $f$ nite loops.

From a model theoretic point of view it is easy to defi ne a semantics that derives " not $P(t)$ ": we could just take all minimal Herbrand models as the intended semantics. Of course, this does not change the general undecidability.

For the exact terminology, defi nitions and results presented in this section we refer the interested reader to the following interesting overviews [Schlipf, 1990; Schlipf, 1992; Cadoli and Schaerf, 1993]. Further results are contained in [Eiter et al., 1993; Sacca, 1993; Chomicki and Subrahmanian, 1990; Eiter and Gottlob, 1993b].

While Table 2 on the next page treats the complexity Table 3 on page 33 treats the expressibility problem. Some general explanations are appropriate. 


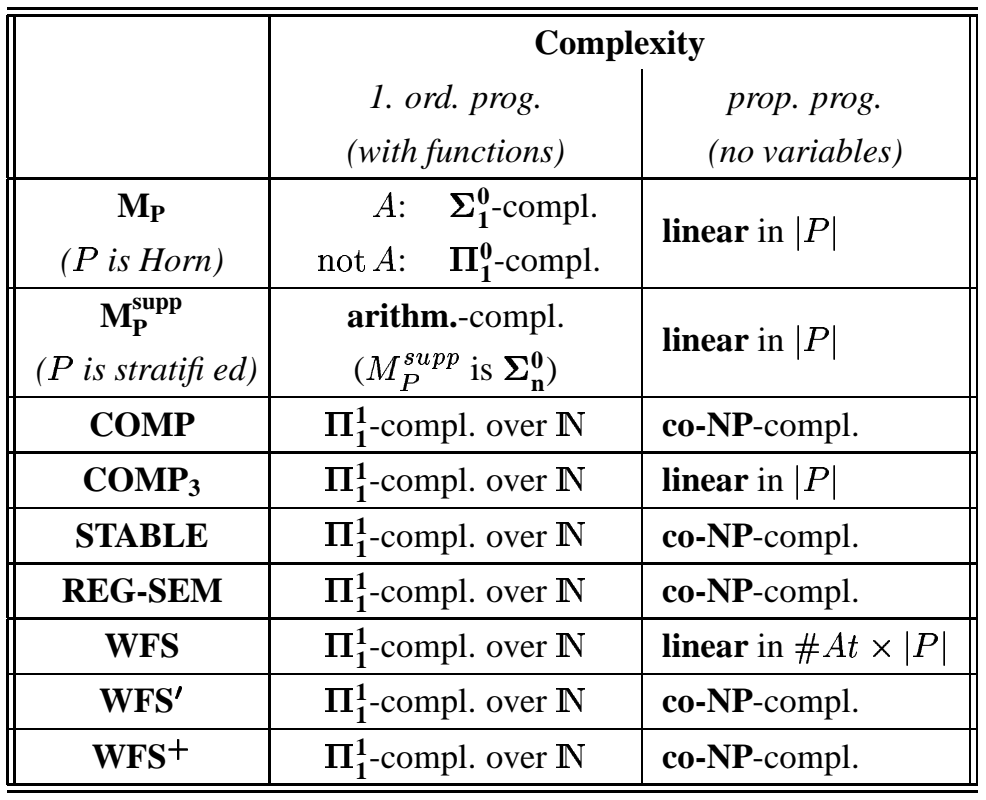

Table 2. Complexity of Non-Disjunctive Semantics

Table 2

We consider the complexity of deciding if a given ground atom or default-atom is contained in the respective semantics (i.e. if it is true in all intended models).

For the fir rst column, we consider arbitrary fi rst-order programs with function symbols. We therefore get undecidability results of varying strength. Since we restrict to Herbrand models, we can assume (by standard recursive encoding techniques, like Gödel-numberings) that all models have universes which are subsets of the natural numbers $\mathbb{N}$. The completeness results mean that for every set of the respective complexity class there is a program that defi nes this set under the respective sceptical semantics. Unless indicated otherwise, there is no difference between deciding ground atoms or ground negated atoms.

For the second column, we consider propositional programs. Hence we get decidable problems of various degrees. We denote by $|P|$ the total length of the program and by \#At the number of distinct proposition letters in $P$. See also [BenEliyahu and Dechter, 1992; Schlipf, 1992; Imielinski, 1991; Marek et al., 1992; Witteveen, 1991b] for more results on the complexity of propositional programs. 
Table 3

Here we consider the expressibility (or expressive power) of fi rst order programs without function symbols. The idea is to distinguish between EDB-relations (relations that do not appear in the head of a program) and IDB-relations (which are contained in some heads). For a given program $P$ we can view any instance $\mathcal{D}$ of the (fi nite) EDB-relations as an input argument and then compute the (fi nite) IDB-relations (the output) under the respective sceptical semantics. So we are asking

What are the relations expressible with logic programs under certain semantics?

Roughly speaking, a relation $R$ over fi nite EDB's $\mathcal{D}$ (i.e. for every fi nite $\mathcal{D}$ is associated a relation $R^{\mathcal{D}}$ on $\mathcal{D}$ ) is expressible if there is a program $P$ containing an IDB-symbol $r \mathrm{~s}$. t. for every relational database $\mathcal{D}$ and tuple $\underline{t}$ corresponding to $r$ :

$$
r(\underline{t}) \in S E M(P+\mathcal{D}) \text { if and only if } R(\underline{t}) \text { holds in } \mathcal{D} .
$$

This is the classical notion of expressibility ([Schlipf, 1990; Eiter et al., 1993]).

We are in particular interested to express all relations of some complexity class (note that the complexity is always with respect to the fi nite relational database as input, the program is fixed). It is well-known that the relations inductively defi nable over $\mathcal{D}$, we denote them by $\operatorname{IND}(\mathcal{D})$ (or simply IND to avoid the explicit occurrence of the EDB), is a strict subclass of the relations that are polynomial over $\mathcal{D}$ (see [Barwise, 1975; Moschovakis, 1974; Gurevich, 1988]).

It is worth noting that in the general predicate logic case, all semantics are highly undecidable. The entries for comp and comp $\mathrm{p}_{3}$ are to be understood as restricted to Herbrand models.

In the propositional case, WFS is of quadratic complexity (a folklore resultfor a proof see [Witteveen, 1991a]), while STABLE is co-NP-complete. The low complexity of WFS can be traced back to Dowling and Gallier's result whereby satisfi ability of Horn clauses can be tested in linear time (Dowling and Gallier, 1984]). In Dowling and Gallier's approach it is actually a minimal model of a Horn theory that is computed in linear time. Since minimal models of Horn theories are equivalent to closures of rules without negation the result is directly applicable to well-founded semantics for logic programs with default-atoms.

As far as expressibility is concerned, STABLE is more expressive: all co-NPrelations can be expressed, while WFS can only describe all inductively defi nable relations. As an example, STABLE can express the satisfi ability problem. WFS is not able to do this (unless the polynomial hierachy collapses).

\section{ADDING EXPLICIT NEGATION}

So far we have considered programs with one special type of negation, namely default negation. Default negation is particularly useful in domains where complete 


\begin{tabular}{||c|c||}
\hline \hline & $\begin{array}{c}\text { Expressibility } \\
\text { 1. ord. prog. } \\
\text { (no functions) }\end{array}$ \\
\hline $\begin{array}{c}\mathbf{M}_{\mathbf{P}} \\
(P \text { is Horn) }\end{array}$ & $\subset \mathbf{I N D}$ (thus $\subset \mathbf{P})$ \\
\hline $\mathbf{C O M P}$ & $=\mathbf{c o}-\mathbf{N P}$ \\
\hline $\mathbf{C O M P}_{3}$ & $=\mathbf{I N D}$ (thus $\subset \mathbf{P})$ \\
\hline $\mathbf{S T A B L E}$ & $=\mathbf{c o}-\mathbf{N P}$ \\
\hline REG-SEM $^{\mathbf{P}}$ & $=\mathbf{\Pi}_{\mathbf{2}}$ \\
\hline $\mathbf{W F S}^{\prime}$ & $=\mathbf{I N D}$ (thus $\subset \mathbf{P})$ \\
\hline $\mathbf{W F S}^{\prime}$ & $=\mathbf{I N D}$ (thus $\subset \mathbf{P}$ ) \\
\hline $\mathbf{W F S}^{+}$ & $=\mathbf{I N D}$ (thus $\subset \mathbf{P}$ ) \\
\hline \hline
\end{tabular}

Table 3. Expressibility of Non-Disjunctive Semantics

positive information can be obtained. For instance, if one wants to represent flight connections from Budapest to the US it is very convenient to represent all existing flights and to let default negation handle the derivation of negative information. There are domains, however, where the lack of positive information cannot be assumed to support (or support with enough strength) that this information is false. In such domains it becomes important to distinguish between cases where a query does not succeed and cases where the negated query succeeds. The following example was used by McCarthy to illustrate the issue. Assume one wants to represent the rule: cross the railroad tracks if no train is approaching. The straightforward representation of this rule with default negation would be

$$
\text { crosstracks } \leftarrow \text { not train }
$$

It seems obvious that in many practical settings the use of such a rule would not lead to intended behaviour, in fact it might even have disasterous consequences. What seems to be needed here is the possibility of using a different negation symbol representing a stronger form of negation. This new negation-we will call it explicit negation-should be true only if the corresponding negated literal can actually be derived. We will use the classical negation symbol $\neg$ to represent explicit negation. The track crossing rule will be represented as

$$
\text { crosstracks } \leftarrow \neg \text { train }
$$

The idea is that this latter rule will only be applicable if $\neg$ train has been proved, contrary to the fir rst rule which is applicable whenever train is not provable. 
In the next subsection we will shortly discuss that explicit negation is not (or should not be) classical negation and how it should interfere with default negation. In the two following subsections we will generalize the semantics STABLE and WFS, respectively, to programs with explicit negation.

\subsection{Explicit vs. Classical and Strong Negation}

First we defi ne the language we are using more precisely.

Definition 4.1 (Extended Logic Program).

An extended logic program consists of rules of the form

$$
c \leftarrow a_{1}, \ldots, a_{n}, \operatorname{not} b_{1}, \ldots, \operatorname{not} b_{m}
$$

where the $a_{i}, b_{j}$ and $c$ are literals, i.e., either propositional atoms or such atoms preceded by the classical negation sign. The symbol "not" denotes negation by failure (default negation), “ $\neg "$ denotes explicit negation.

We have already motivated the need of a second kind of negation " $\neg$ " different from "not". What should the semantics of " $\neg$ " be? Should it be just like in classical logic? Note that classical negation satisfi es the law of excluded middle

$$
A \vee \neg A \text {. }
$$

The following example taken from [Alferes et al., 1996] shows that classical negation is sometimes inappropriate for KR-tasks.

Example 4.1 (Behaviour of Classical Negation).

Suppose an employer has several candidates that apply for a job. Some of them are clearly qualifi ed while others are not. But there may also be some candidates whose qualifi cations are not clear and who should therefore be interviewed in order to find out about their qualifi cations. If we express the situation by

$$
\text { hire }(X) \leftarrow \text { qualified }(X) \text { and } \operatorname{reject}(X) \leftarrow \neg \text { qualified }(X)
$$

then, interpreting “ $\neg$ " as classical negation, we are forced to derive that every candidate must either be hired or rejected! There is no room for those that should be interviewed. Also, applying the law of excluded middle has a highly nonconstructive flavor.

Let us now consider again the example crosstracks $\leftarrow \neg$ train from the beginning of this section. Suppose that we replace $\neg$ train by free_track. We obtain

$$
\text { crosstracks } \leftarrow \text { free_track. }
$$

From this program, "not crosstracks" will be derivable for any semantics. Therefore we should make sure that "not crosstracks" is also derivable from

crosstracks $\leftarrow \neg$ train 
After all, the second program is obtained from the fi rst one by a simple syntactic operation. This means we have to make sure that default negation "not" treats positive and negated atoms symmetrically.

Such a negation, we will call it explicit will be introduced in the next two subsections. Note that Gelfond/Lifschitz called the negation they introduced in their stable semantics classical, although it is not classical in the sense that we just discussed. Sometimes explicit negation is also called strong negation and denotes still a variant of our explicit negation. In [Alferes et al., 1996] the authors introduce both a strong and explicit negation and discuss their relation with classical and default negation at length.

\subsection{STABLE for Extended Logic Programs}

The extension of STABLE to extended logic programs is based on the notion of answer sets which generalize the original notion of stable models in a rather straightforward manner. Let us fi rst introduce some useful notation. We say a rule $r=c \leftarrow a_{1}, \ldots, a_{n}$, not $b_{1}, \ldots$, not $b_{m} \in P$ is defeated by a literal $l$ iff $l=b_{i}$ for some $i \in\{1, \ldots, m\}$. We say $r$ is defeated by a set of literals $X$ if $X$ contains at least one literal that defeats $r$. Furthermore, we call the rule obtained by deleting default negated antecedents from $r$ the monotonic counterpart of $r$ and denote it with $\operatorname{Mon}(r)$. We also apply Mon to sets of rules with the obvious meaning.

Definition 4.2 ( $X$-reduct).

Let $P$ be an extended logic program, $X$ a set of literals. The $X$-reduct of $P$ is the set of rules

$$
P^{X}:=\{\operatorname{Mon}(r): r \text { not defeated by } X\} .
$$

Note that the only difference between the defi nition of $P^{N}$ in Section 3.4 and this defi nition is that the new one handles literals and not only than atoms.

The defi nition of stable models (Defi nition 3.4) was based on the minimal model of the reduct. Since the reduct now may contain explicit negation we need a different notion here, namely a notion of consequence:

Definition 4.3 (Consequences of Rules).

Let $R$ be a set of rules without default negation. $\operatorname{Cn}(R)$ denotes the smallest set of literals that is

1. closed under $R$, and

2. logically closed, i.e., either consistent or equal to the set of all literals.

Definition $4.4\left(\gamma_{P}\right)$.

Let $P$ be a logic program, $X$ a set of literals. Defi ne an operator $\gamma_{P}$ as follows:

$$
\gamma_{P}(X)=\operatorname{Cn}\left(P^{X}\right)
$$

$X$ is an answer set of $P$ iff $X=\gamma_{P}(X)$. 
A literal $l$ is a consequence of a program $P$ under the new semantics, denoted $l \in S T A B L E(P)$, iff $l$ is contained in all answer sets of $P$.

It is not diffi cult to see that for programs without explicit negation stable models and answer sets coincide. Here is an example involving both types of negation. The example describes the strategy of a certain college for awarding scholarships to its students. It is taken from [Baral and Gelfond, 1994]:

$$
\begin{aligned}
& P_{e l}:(1) \text { eligible }(x) \leftarrow \operatorname{high} G P A(x) \\
& \text { (2) eligible }(x) \leftarrow \text { minority }(x) \text {, fairGPA(x) } \\
& \text { (3) } \neg \text { eligible }(x) \leftarrow \neg \text { fairGPA(x), ᄀhighGPA(x) } \\
& \text { (4) } \operatorname{interview}(x) \leftarrow \operatorname{not} \text { eligible }(x) \text {, not } \neg \text { eligible }(x)
\end{aligned}
$$

Assume in addition to the rules above the following facts about Anne are given:

$$
\text { fairGPA(Anne), ᄀhighGPA(Anne) }
$$

We obtain exactly one answer set, namely

$$
\{\text { fairGPA(Anne), } \neg \text { highGPA(Anne), interview(Anne) }\}
$$

Anne will thus be interviewed before a decision about her eligibility is made. If we use the above rules together with the facts

$$
\text { minority (Mike), fairGPA(Mark) }
$$

then the program entails eligible $(M i k e)$.

The following results are taken from [Lifschitz, 1996]:

\section{Lemma 4.1 (Program Types).}

Let $P$ be an extended logic program. $P$ satisfi es exactly one of the following conditions:

- $P$ has no answer sets,

- the only answer set for $P$ is 1 Lit,

- $P$ has an answer set, and all its answer sets are consistent.

A program is consistent if the set of its consequences is consistent, and inconsistent otherwise. The former corresponds to the fi rst two cases listed in the proposition, the latter to the third case.

We say that a set $X$ of literals is supported by $P$ if, for each literal $l \in X$, there exists a rule $l \leftarrow a_{1}, \ldots, a_{n}$, not $b_{1}, \ldots$, not $b_{m}$ in $P$ such that

1. $\left\{a_{1}, \ldots, a_{n}\right\} \subseteq X$, and

2. $\left\{b_{1}, \ldots, b_{m}\right\} \cap X=\emptyset$. 
Lemma 4.2 (Properties of answer sets).

Let $P$ be an extended logic program. The following properties hold:

- Any consistent answer set for $P$ is supported by $P$.

- If $X$ and $Y$ are answer sets of $P$ and $X \subseteq Y$ then $X=Y$.

- Each element of a consistent answer set of $P$ is a head literal ${ }^{13}$ of $P$.

From the last property it follows immediately that every consequence of $P$ is a head literal of $P$ whenever $P$ is consistent. We would fi nally like to mention the following theorem:

Theorem 4.3 (Head Consistency).

If the set of head literals of an extended program $P$ is consistent then every answer set of $P$ is consistent.

Note that a program satisfying the conditions of the last theorem can still be inconsistent since it may have no answer set at all.

It should be noted that extended logic programs under answer set semantics can be reduced to general logic programs as follows: for any predicate $p$ occurring in a program $P$ we introduce a new predicate symbol $p^{\prime}$ of the same arity representing the explicit negation of $p$. We then replace each occurrence of $\neg p$ in the program with $p^{\prime}$, thus obtaining the general logic program $P^{\prime}$. It can be proved that a consistent set of literals $S$ is an answer set of $P$ iff the set $S^{\prime}$ is a stable model of $P^{\prime}$, where $S^{\prime}$ is obtained from $S$ by replacing $\neg p$ with $p^{\prime}$.

\subsection{WFS for Extended Logic Programs}

We now show how the second major semantics for general logic programs, WFS, can be extended to logic programs with explicit negation. For our purposes the characterization of WFS given in Theorem 3.6 on page 30 will be useful. WFS is based on a particular three-valued model. To simplify our presentation in this section we will restrict ourselves to the literals which are true in this three-valued model. The literals which are false will be left implicit. They can be added in a canonical way as follows: let $T$, the set of true literals, be defi ned as the least fi xpoint of a monotone operator composed of two antimonotone operators $o p_{1} o p_{2}$. Then the literals which are false in the three-valued model are exactly those which are not contained in $O p_{2}(T)$. Given this canonical extension to the full three-valued model we can safely leave the false literals implicit from now on.

We will fi rst present a formulation which can be found in various papers, e.g. [Baral and Gelfond, 1994; Lifschitz, 1996]. We then slightly modify this formulation to obtain stronger results. We fi nally discuss a further modifi cation by Pereira and Alferes.

\footnotetext{
${ }^{13} \mathrm{~A}$ head literal of a program $P$ is the head of a rule of $P$ (see also Principle 1 on page 7 and Definition 6.3 on page 51 ).
} 
Like answer set semantics well-founded semantics for extended logic programs can be based on the operator $\gamma_{P}$. However, the operator is used in a totally different way. Since $\gamma_{P}$ is anti-monotone the operator $\Gamma_{P}=\left(\gamma_{P}\right)^{2}$ is monotone. According to the famous Knaster-Tarski theorem [Tarski, 1955] every monotone operator has a least fi xpoint. We can thus defi ne

\section{Definition 4.5 (WFS for extended programs).}

Let $P$ be an extended logic program. The set of well-founded conclusions of $P$, denoted $W F S(P)$, is the least fi xpoint of $\Gamma_{P}$.

The fi xpoint can be approached from below by iterating $\Gamma_{P}$ on the empty set. In case $P$ is fi nite this iteration is guaranteed to actually reach the fi xpoint.

The intuition behind this use of the operator is as follows: whenever $\gamma_{P}$ is applied to a set of literals $X$ known to be true it produces the set of all literals that are still potentially derivable. Applying it to such a set of potentially derivable literals it produces a set of literals known to be true, often larger than the original set $X$. Starting with the empty set and iterating until the fi xpoint is reached thus produces a set of true literals.

We fi rst want to illustrate this using an example without explicit negation:

$$
\begin{aligned}
& P: \quad(1) \quad b \leftarrow \operatorname{not} a \\
& \text { (2) } c \leftarrow \operatorname{not} b \\
& \text { (3) } e \leftarrow \operatorname{not} d \\
& \text { (4) } d \leftarrow \operatorname{not} e
\end{aligned}
$$

In the beginning we know nothing about derivable literals, i.e., we start with empty set $X$. The $X$-reduct of the program is

$$
\begin{array}{ll}
(1) & b \\
(2) & c \\
(3) & e \\
(4) & d
\end{array}
$$

The set of consequences of this program, or in other words, the literals still considered to be potentially derivable, is thus $\{b, c, d, e\}$. If we now reduce the program with this set we obtain

$$
\text { (1) } b
$$

that is, the first iteration of the two-fold application of $\gamma_{p}$ tells us that $b$ is provable.

If we now use $X=\{b\}$ to continue the iteration we obtain the reduced program

$$
\begin{array}{ll}
\text { (1) } & b \\
\text { (3) } & e \\
\text { (4) } & d
\end{array}
$$

that is $\{b, d, e\}$ is the current set of potential conclusions. Using this set to reduce the program gives us again

(1) $b$ 
We thus have reached the least fi xpoint of $\gamma_{P}^{2}$ and $b$ is the single literal provable under WFS.

It can be shown that every well-founded conclusion is a conclusion under the answer set semantics. Well-founded semantics can thus be viewed as an approximation of answer set semantics.

Unfortunately it turns out that for many programs the set of well-founded conclusions as defi ned in Defi nition 4.5 is extremely small and provides a very poor approximation of answer set semantics. Consider the following program $P_{0}$ which has also been discussed by Baral and Gelfond [Baral and Gelfond, 1994]:

$$
\begin{aligned}
& P_{0}: \quad(1) \quad b \quad \leftarrow \operatorname{not} \neg b \\
& \text { (2) } a \leftarrow \operatorname{not} \neg a \\
& \text { (3) } \neg a \leftarrow \operatorname{not} a
\end{aligned}
$$

The set of well-founded conclusions is empty since $\gamma_{P_{0}}(\emptyset)$ equals Lit, the set of all literals, and the $L i t$-reduct of $P_{0}$ contains no rule at all. This is surprising since, intuitively, the conflict between (2) and (3) has nothing to do with $\neg b$ and $b$.

This problem arises whenever the following conditions hold:

1. a complementary pair of literals is provable from the monotonic counterparts of the rules of a program $P$, and

2. there is at least one proof for each of the complementary literals whose rules are not defeated by $\operatorname{Cn}\left(P^{\prime}\right)$, where $P^{\prime}$ consists of the "strict" rules in $P$, i.e., those without negation as failure.

In this case well-founded semantics concludes $l$ iff $l \in \operatorname{Cn}\left(P^{\prime}\right)$. It should be obvious that such a situation is not just a rare limiting case. To the contrary, it can be expected that many commonsense knowledge bases will give rise to such undesired behaviour. Let us consider again our Example 2.4 on page 12 from Section 2.

$$
\begin{aligned}
& \text { (1) } \operatorname{fly}(x) \leftarrow \operatorname{not} \neg f l y(x), \operatorname{bird}(x) \\
& \text { (2) } \neg \operatorname{fly}(x) \leftarrow \operatorname{not} f l y(x), \operatorname{penguin}(x)
\end{aligned}
$$

Assume further that the knowledge base contains the information that Tweety is a penguin bird. Now if neither $f l y($ Tweety) nor $\neg f l y(T$ weety) follows from strict rules in the knowledge base we are in the same situation as with $P_{0}$ : well-founded semantics does not draw any "defeasible" conclusion, i.e. a conclusion derived from a rule with default negation in the body, at all.

We want to show that a minor reformulation of the fi xpoint operator can overcome this weakness and leads to better results. Consider the following operator

$$
\gamma_{P}^{\star}(X)=C l\left(P^{X}\right)
$$

where $C l(R)$ denotes the minimal set of literals closed under the (classical) rules $R$. $C l(R)$ is thus like $\operatorname{Cn}(R)$ without the requirement of logical closedness. Now 
defi ne

$$
\Gamma_{P}^{\star}(X)=\gamma_{P}\left(\gamma_{P}^{\star}(X)\right)
$$

Again we iterate on the empty set to obtain the well-founded conclusions of a program $P$ which we will denote $W F S^{\star}(P)$.

Consider the effects of this modifi cation on our example $P_{0}$ :

$$
\gamma_{P_{0}}^{\star}(\emptyset)=\{a, \neg a, b\} .
$$

Rule (1) is contained in the $\{a, \neg a, b\}$-reduct of $P_{0}$ and thus $\Gamma_{P_{0}}^{\star}(\emptyset)=\{b\}$. Since $b$ is also the only literal contained in all answer sets of $P_{0}$ our approximation actually coincides with answer set semantics in this case.

In the Tweety example both $f l y($ Tweety $)$ and $\neg f l y($ Tweety $)$ are provable from the $\emptyset$-reduct of the knowledge base. However, this has no influence on whether a rule not containing the default negation of one of these two literals in the body is used to produce $\gamma_{P}^{\star}(\emptyset)$ or not. The effect of the conflicting information about Tweety's flying ability is thus kept local and does not have the disastrous consequences it has in the original formulation of well-founded semantics.

It is not diffi cult to see that the new monotone operator is equivalent to the original one whenever $P$ does not contain negation as failure. In this case the $X$-reduct of $P$, for arbitrary $X$, is equivalent to $P$ and for this reason it does not make any difference whether to use $\gamma_{P}$ or $\gamma_{P}^{\star}$ as the operator to be applied fi rst in the defi nition of $\Gamma_{P}$. The same is obviously true for programs without classical negation: for such programs $\mathrm{Cn}$ can never produce complementary pairs of literals and for this reason the logical closedness condition is obsolete.

In the general case the new operator produces more conclusions than the original one:

Lemma 4.4. Let $P$ be an extended logic program. For an arbitrary set of literals $X$ we have

$$
\Gamma_{P}(X) \subseteq \Gamma_{P}^{\star}(X) .
$$

It can also be shown that the new operator produces no unwanted results, i.e., that our new semantics can still be viewed as an approximation of answer set semantics.

Lemma 4.5. Let $P$ be an extended logic program. $W F S^{\star}$ is correct wrt. $S T A B L E$, i.e., $l \in W F S^{\star}(P)$ implies $l \in S T A B L E(P)$.

An alternative, somewhat stronger approach, was developed by Pereira and Alferes [Pereira and Alferes, 1992; Alferes and Pereira, 1995; Alferes and Pereira, 1996], the semantics WFSX. This semantics implements the intuition that a literal with default negation should be derivable from the corresponding explicitly negated literal. The authors call this the coherence principle. To satisfy the principle they use the seminormal version of a program $P$, denoted $S(P)$, which is obtained from $P$ by replacing each rule

$$
c \leftarrow a_{1}, \ldots, a_{n}, \operatorname{not} b_{1}, \ldots, \operatorname{not} b_{m}
$$


by the rule

$$
c \leftarrow a_{1}, \ldots, a_{n}, \operatorname{not} b_{1}, \ldots, \operatorname{not} b_{m}, \operatorname{not}-c
$$

where $-c$ is the complement of $c$, i.e. $\neg c$ if $c$ is an atom and $a$ if $c=\neg a$. Based on this notion Pereira and Alferes consider the following monotone operator:

$$
\Omega_{P}(X)=\gamma_{P}^{\star} \gamma_{S(P)}^{\star}(X)
$$

The use of the seminormal version of the program in the first application of $\gamma^{\star}$ guarantees that a literal $l$ is not considered a potential conclusion whenever the complementary literal is already known to be true. In the general case $S(P)^{X}$ contains fewer rules than $P^{X}$. Therefore, fewer literals are considered as potential conclusions and thus more conclusions are obtained in each iteration of the monotone operator. Here is an example [Baral and Gelfond, 1994]:

$$
\begin{array}{rlll}
P_{W F S X}: & (1) & a & \leftarrow \operatorname{not} b \\
& (2) \quad b & \leftarrow \operatorname{not} a \\
& (3) \quad \neg a & \leftarrow
\end{array}
$$

The original version of WFS does not conclude $b$. In WFSX the set $X=\{\neg a\}$ is obtained after the fi rst iteration of the monotone operator. Since rule (1) is not contained in the $X$-reduct of the seminormal version of the program the monotonic counterpart of (2) produces $b$ after the second iteration.

\section{ADDING PREFERENCES}

In this section we describe how preferences among rules can be taken into account in logic programs with two types of negation. The basic idea is the following: in case of a conflict between rules preferences are used to break ties wherever possible. For programs under answer set semantics this means that some of the answer sets are preferred, the others disregarded. The conclusions of a prioritized logic program are then defi ned as the intersection of all preferred answer sets. In the general case this leads to a larger set of conclusions.

For well-founded semantics we will take preferences into account by modifying the monotone operator whose least fi xpoint defi nes the well-founded conclusions. Again more well-founded conclusions will be generated than without preferences.

We fi rst give some motivation in Section 5.1. We then describe how preferred answer sets can be defi ned on the basis of preferences among rules. Our presentation of this subsection is based on [Brewka and Eiter, 1999]. Section 5.3 adds preferences to well-founded semantics and is based on [Brewka, 1996b] as well as [Brewka, 2001]. Subsection 5.4 illustrates the expressiveness of our approach using a decision making example.

Note that we use $r_{1}<r_{2}$ to express that $r_{1}$ is preferred over $r_{2}$, that is the smaller rules are the better ones in this section. 


\subsection{Motivation}

Preferences among defaults play a crucial role in nonmonotonic reasoning. One source of preferences that has been studied intensively is specifi city[Poole, 1985; Touretzky, 1986; Touretzky et al., 1991]—we already discussed it in Example 2.4 on page 12. In case of a conflict between defaults we tend to prefer the more specifi c one since this default provides more reliable information. E.g., if we know that students are adults, adults are normally employed, students are normally not employed, we want to conclude "Peter is not employed" from the information that Peter is a student, thus preferring the student default over the conflicting adult default.

Specifi city is an important source of preferences, but not the only one, and at least in some applications not necessarily the most important one. In the legal domain it may, for instance, be the case that a more general rule is preferred since it represents federal law as opposed to state law [Prakken, 1993]. In these cases preferences may be based on some basic principles regulating how conflicts among rules are to be resolved.

Also in other application domains, like model based diagnosis or confi guration, preferences play a fundamental role. Model based diagnosis uses logical descriptions of the normal behaviour of components of a device together with a logical description of the actually observed behaviour. One tries to assume normal behaviour for as many components as possible. A diagnosis corresponds to a set of components for which these normalcy assumptions lead to inconsistency. Very often a large number of possible diagnoses is obtained. In real life some components are less reliable than others. To eliminate less plausible diagnoses one can give the normalcy assumptions for reliable components of higher priority.

In confi guration tasks it is often impossible to achieve all of the design goals. Often one can distinguish more important goals from less important ones. To construct the best possible confi gurations goals then have to be represented as defaults with different preferences according to their desirability.

Preferences also turn out to be relevant for reasoning about action. For instance, Kakas, Miller and Toni developed an approach where preferences between different types of rules (inertia rules, effect rules etc.) are used to model commonsense reasoning involving actions [Kakas et al., 2001].

Prioritized versions for most of the existing nonmonotonic formalisms have been proposed, e.g., prioritized circumscription [Lifschitz, 1994], hierarchic autoepistemic logic [Konolige, 1988], prioritized default logic [Marek and Truszczyński, 1993; Brewka, 1994; Baader and Hollunder, 1995], prioritized theory revision [Benferhat et al., 1993; Nebel, 1998], or prioritized abduction [Eiter and Gottlob, 1995]. Somewhat surprisingly, at least for some time preferences have received less attention in logic programming. This may be explained by the fact that for a long period, logic programming was mainly conceived as a logical paradigm for declarative programming, and to a less extent as a tool for knowledge representation and reasoning. However, it has become evident that logic programming can 
serve as a powerful framework for knowledge representation, cf. [Gelfond, 1994; Baral and Gelfond, 1994]. If logic programming wants to successfully stand this challenge, it must provide the features which have been recognized as indispensable in the context of knowledge representation. One such feature is the possibility to handle specifi city and priority of knowledge.

\subsection{Preferred Answer Sets}

In this section we will briefly describe the approach developed in [Brewka and Eiter, 1999]. For a more general treatment, more detail and more motivation consult the original paper.

Let us fi rst defi ne what we mean by a prioritized logic program. For simplicity we consider only propositional programs in this and the following section, i.e. we assume that programs are ground and fi nite:

\section{Definition 5.1 (Prioritized Program).}

A (propositional) prioritized logic program is a pair

$$
\mathcal{R}=(R,<)
$$

where $R$ is a fi nite set of ground rules and < is a strict partial order on $P$.

The treatment of partially ordered prioritized programs is reduced to that of totally ordered programs as follows:

\section{Definition 5.2 (Preferred Answer Set).}

Let $\mathcal{R}=(R,<)$ be a prioritized logic program. $A$ is a preferred answer set of $\mathcal{R}$ iff $A$ is a preferred answer set of $\mathcal{R}^{\prime}=\left(R,<^{\prime}\right)$, where $<^{\prime}$ is an arbitrary total order on $R$ extending <.

What remains to be defi ned then are preferred answer sets for totally ordered programs. The approach presented here was originally introduced using a new reduct which can be viewed as dual to the Gelfond-Lifschitz reduct. Rather than eliminating negation based on a given set of literals, as the Gelfond-Lifschitz reduct, the new reduct eliminates prerequisites. The idea is that the treatment of preferences can thus be reduced to prerequisite-free programs which can be handled much easier.

The exact defi nitions are somewhat involved. For the purposes of this overview it is suffi cient to give a characterization of preferred answer sets which is based on Proposition 5.1 in [Brewka and Eiter, 1999]. We use the following notation: pre $(r)$ denotes the set of antecedents of the rule $r$ are which are not default negated (called prerequisites of $r$ ), head $(r)$ denotes the head of $r$, and a literal $l$ defeats a rule $r$ if not $l$ appears in the body of $r . r$ is said to be a generating rule of answer set $A$ iff $p r e(r) \in A$ and $r$ is not defeated by any literal in $A$.

PROPOSITION 1 (Preferred Answer Sets and Generating Rules).

Let $\mathcal{R}=(R,<)$ be a totally prioritized logic program and let $A$ be an answer set of $R$. A is a preferred answer set of $\mathcal{R}$ if and only if for each rule $r \in R$ such that 
pre $(r) \subseteq A$ and head $(r) \notin A$ there is a generating rule $r^{\prime} \in R$ such that $r^{\prime}<r$ and head $\left(r^{\prime}\right)$ defeats $r$.

Here is a simple example illustrating this characterization of preferred answer sets:

(1) $b \leftarrow \operatorname{not} a$

(2) $a \leftarrow \operatorname{not} b$

Aussume $(1)<(2)$. The program has two answer sets, $A_{1}=\{b\}$ and $A_{2}=\{a\}$, the former generated by rule (1), the latter by (2). Rule (2) was not applied in $A_{1}$ although its prerequisites are in $A_{1}$. This is perfectly ok since rule (1) which is a generating rule of higher priority defeats (2). $A_{1}$ is thus a preferred answer set.

Now consider $A_{2}$. Rule (1) was not applied, yet there is no generating rule of $A_{2}$ defeating (1) which is of higher priority than (1). For this reason, $A_{2}$ is not a preferred answer set.

In [Brewka and Eiter, 1999] it was proven that the approach presented here, contrary to several competing approaches, satisfi es two natural principles of preference handling in rule based systems. The paper also contains a discussion of many alternative approaches found in the literature. A more recent comparison of some of these approaches can be found in [Schaub and Wang, 2001].

\subsection{Prioritized WFS}

We now show how preferences can adequately be added to well-founded semantics. We will use the variant of WFS for extended logic programs which is based on the operator $\gamma^{\star}$ as described in Section 4.3.

For this section a minor reformulation turns out to be convenient. Instead of using the monotonic counterparts of undefeated rules we will work with the original rules and extend the defi nitions of the consequence operator $\mathrm{Cn}$ and the closure operator $\mathrm{Cl}$ accordingly. We simply require that default negated preconditions be neglected, i.e., for an arbitrary set of rules $P$ possibly containing default negation we defi ne $\mathrm{Cn}(P)=\mathrm{Cn}(\operatorname{Mon}(P))$ and $\mathrm{Cl}(P)=\mathrm{Cl}(\operatorname{Mon}(P))$. We can now equivalently characterize $\gamma_{P}$ and $\gamma_{P}^{\star}$ by the equations

$$
\begin{aligned}
& \gamma_{P}(X)=\operatorname{Cn}\left(P_{X}\right) \\
& \gamma_{P}^{\star}(X)=\operatorname{Cl}\left(P_{X}\right)
\end{aligned}
$$

where $P_{X}$ denotes the set of rules not defeated by $X$.

As mentioned earlier, the intuition behind well-founded semantics can be described as follows: given a set of literals $S$ already known to be derivable, $\gamma^{*}(S)$ produces a set of potential conclusions which still might defeat rules in $P$. The conclusions of rules not defeated by any of the potential defeaters are clearly derivable. Starting with the empty set, we thus generate larger and larger sets $S$ until a fi xpoint is reached. The following terminology - somewhat influenced by argumentation theory - reflects this intuition: 
Definition 5.3 (Defeated Rules).

Let $P$ be an extended logic program.

- A literal $l$ is an $S$-potential defeater iff $l$ is in the closure of the rules in $P$ not defeated by $S$.

- A rule $r$ is $S$-undefeatable iff $r$ is not defeated by any $S$-potential defeater.

- A literal $l$ is $S$-derivable iff $l$ is a consequence of the $S$-undefeatable rules in $P$.

It is obvious that $l$ is $S$-derivable iff $l \in \gamma\left(\gamma^{*}(S)\right)$. The least fi xpoint of $\gamma \gamma *$, $W F S(P)$, can thus equivalently be characterized as the least fi xpoint of the operator which, given a set $S$, produces the set of $S$-derivable literals. It turns out that this reformulation is most adequate for introducing preferences.

To take preferences into account we fi rst introduce a notion of dominance. Intuitively, a rule $r$ dominates a rule $r^{\prime}$ in the context of a set of literals $S$ if $r$ has higher priority and if the application of $r$ in context $S$ actually defeats $r^{\prime}$. As pointed out in [Brewka, 1996a] the second condition is necessary to guarantee that prioritized well-founded semantics is an extension of well-founded semantics. Here is the formal defi nition

Definition 5.4 ( $S$-dominates $\left.r^{\prime}\right)$.

Let $r$ and $r^{\prime}$ be rules, $S$ a set of literals. We say $r S$-dominates $r^{\prime}$ iff

1. $r<r^{\prime}$, and

2. $\mathrm{Cl}(\{r\} \cup\{s: s$ is $S$-undefeatable $\})$ defeats $r^{\prime}$.

For the case of prioritized programs Defi nition 5.3 becomes

\section{Definition 5.5 (Potential Defeater).}

Let $(P,<)$ be a prioritized logic program.

- A literal $l$ is an $S$-potential $r$-defeater iff $l$ is in the closure of rules in $P$ which are (1) not defeated by $S$ and (2) not $S$-dominated by $r$.

- A rule $r$ is $S$-safe iff $r$ is not defeated by any $S$-potential $r$-defeater.

- A literal $l$ is $S$-derivable iff $l$ is a consequence of the set of $S$-safe rules in $P$.

The defi nition for prioritized logic programs differs from the one for non-prioritized programs in two respects. Firstly, there is not a single set of potential defeaters for all rules but each rule $r$ has its own set of potential defeaters. Secondly, the rules which are used to derive potential defeaters must satisfy an additional condition: to potentially defeat $r$ a rule must not be dominated by $r$ in context $S$. Since fewer rules can be used to derive potential defeaters for a rule the safe rules are a superset 
of the undefeatable rules. We thus obtain more derivable literals. For the special case where $<$ is empty the two defi nitions of $S$-derivable clearly coincide.

The set of $S$-derivable literals grows monotonically with $S$. We thus can start as usual with the empty set of literals and iterate the computation of $S$-derivable formulae until a fi xpoint is reached.

Here is a small example illustrating the defi nition.

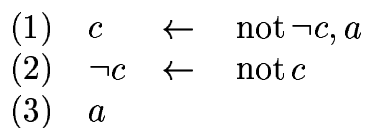

Let $(1)<(2)$. Clearly, rule (3) is $\emptyset$-safe since there is no way of defeating a rule without default negation. But also (1) is $\emptyset$-safe since the closure of (1) together with the $\emptyset$-undefeatable rule (3) defeats (2) and thus (1) $\emptyset$-dominates (2). Therefore, the set of $\emptyset$-derivable literals is $\{c, a\}$. This set is already the least fi xpoint. Note that $c$ is not a well-founded conclusion of the program without priorities.

\subsection{An Application: Qualitative Decision Making}

In this section we want to discuss a somewhat more realistic example and show how prioritized logic programs can be used for qualitative decision making. Although we considered fi nite propositional programs only in the last two subsections we will, for simplicity, use rules with variables in this subsection. Since we do not need functions the Herbrand universe is fi nite and we clearly do not go beyond fi nite propositional programs. Note that $r_{1}<r_{2}$ for rules with variables means that all ground instantiations of $r_{1}$ are preferred over all ground instantiations of $r_{2}$. We also write $R_{1}<R_{2}$ for sets of rules $R_{1}$ and $R_{2}$ to express that each element of $R_{1}$ is preferred over each element of $R_{2}$.

Assume you want to buy a car. You have collected the following information about different types of cars:

$$
\begin{gathered}
\text { expensive(Chevrolet), safe (Chevrolet), safe(Volvo), } \\
\text { nice (Porsche), fast(Porsche) }
\end{gathered}
$$

Your decision which car to buy is based on different criteria. We can use rules corresponding to normal defaults [Reiter, 1980] in order to represent the properties which you consider relevant. Let's assume you like fast and nice cars. On the other hand, your budget does not allow you to purchase a very expensive car. Moreover, you have to take your wife's wishes into account, and she insists on a car which is known to be safe.

$$
\begin{aligned}
\neg \text { buy }(x) & \leftarrow \operatorname{not} \operatorname{buy}(x), \text { expensive }(x) \\
\text { buy }(x) & \leftarrow \operatorname{not} \neg \text { buy }(x), \text { safe }(x) \\
\operatorname{buy}(x) & \leftarrow \operatorname{not} \neg \text { buy }(x), \operatorname{nice}(x)
\end{aligned}
$$




$$
b u y(x) \leftarrow \operatorname{not} \neg b u y(x), \text { fast }(x)
$$

Since buying more than one car is out of the question these different decision criteria may obviously lead to conflict, and a preference ordering is necessary. Since there is not much you can do about your restricted budget, rule (1) gets highest preference. Moreover, since your wife is very concerned about safety you better give (2) higher priority than (3) and (4). Since there is tremendous traffi c on highways in your area, (3) is more important to you than (4). This means we have

$$
(1)<(2)<(3)<(4)
$$

We still have to represent that you cannot afford more than one car. A straightforward idea would be to use the rule

$$
\neg b u y(y) \leftarrow b u y(x), x \neq y
$$

with highest priority. Unfortunately, this does not work. The reason is that the high priority of the instances of this rule would allow us to defeat instances of (2), (3) and (4) even if this is not intended. In our example we would obtain two preferred answer sets, the intended one containing buy (Volvo), but also an unintended one (at least from your wife's point of view) containing buy(Porsche). In the latter, the instance of (2) with $x=$ Volvo would be defeated by the instance of $(0)$ with $x=$ Porsche and $y=$ Volvo.

To represent our problem adequately, we have to make sure that the consequences of a certain decision, namely that certain cars are not purchased, do not have higher priority than the decision itself, that is, the decision to buy a specific car. Instead of adding the single rule $(0)$ we therefore represent our criteria for buying a car as pairs of rules. To each rule of the form

(r) $\quad \operatorname{buy}(x) \leftarrow \operatorname{not} \neg b u y(x), c_{1}(x), \ldots, c_{n}(x)$

we add a second one of the form

(r') $\quad \neg b u y(y) \leftarrow c_{1}(x), \ldots, c_{n}(x), \operatorname{buy}(x), x \neq y$

with the same priority as $(r)$. In our example we have to add

(3') $\quad \neg$ buy $(y) \leftarrow \operatorname{nice}(x)$, buy $(x), x \neq y$

(4') $\quad \neg$ buy $(y) \leftarrow \operatorname{fast}(x)$, buy $(x), x \neq y$

and use the following preferences

$$
(1)<\left\{(2),\left(2^{\prime}\right)\right\}<\left\{(3),\left(3^{\prime}\right)\right\}<\left\{(4),\left(4^{\prime}\right)\right\}
$$

Given this information we obtain a single preferred answer set containing buy (Volvo).

This leaves you somewhat dissatisfi ed since you really like the Porsche. You might try to convince your wife that in case a car is both nice and fast you would have heard about any safety problems. That is, you would like to add the following pair of rules: 


$$
\begin{aligned}
(1.5) & \operatorname{buy}(x) & \leftarrow \operatorname{not} \neg b u y(x), \text { not } \neg \text { safe }(x), \operatorname{nice}(x), \text { fast }(x) \\
\left(1.5^{\prime}\right) & \neg b u y(y) & \leftarrow \operatorname{not} \neg \operatorname{safe}(x), \operatorname{nice}(x), \text { fast }(x), \text { buy }(x), x \neq y
\end{aligned}
$$

If your wife accepts this rule with preferences $\left\{(1.5),\left(1.5^{\prime}\right)\right\}<\left\{(2),\left(2^{\prime}\right)\right\}$ you are happy since now the single preferred answer set contains buy(Porsche).

\section{ADDING DISJUNCTION}

In this section we will extend our programs to disjunctive statements. In Knowledge Representation it often occurs that we know $A \vee B \vee C$ without being sure which of these propositions hold. In fact, such a disjunction leaves it open: there might be states in the world where $A$ holds or $B$ or $C$ or any combination thereof. Nevertheless, we can have information that $A$ implies $D$ and $B$ implies $D$ and $C$ implies $D$ from which we would like to derive that $D$ holds for sure. We will see in Section 6.5 that even with disjunctive programs without negation we can already express relations which belong to the second level of the polynomial hierarchy.

Concerning the right semantics for such programs, we are in the same situation as in Section 3-for positive programs there is general agreement while for disjunctive programs with default-negation there exist several competing approaches.

We present in Section 6.1 the generalized closed world assumption introduced by Minker. In Section 6.2 we show that our defi nition of WFS from Section 3.3 immediately carries over to the disjunctive case. The original defi nition of STABLE (Defi nition 3.4 on page 28) also carries over-we present it in Section 6.3. We mention some other attempts to defi ne disjunctive semantics in Section 6.4. Finally we discuss complexity and expressibility in Section 6.5.

\section{$6.1 \quad G C W A$}

GCWA was defi ned by Minker (Minker, 1982]) and can bee seen as a refi ned version of the CWA introduced by Reiter ([Reiter, 1978]):

\section{Definition 6.1 (CWA).}

$$
\mathrm{CWA}(\mathrm{DB})=\mathrm{DB} \cup\{\neg P(t): \mathrm{DB} \not \models P(t)\},
$$

where $P(t)$ is a ground predicate instance.

That is, if a ground term cannot be inferred from the database, its negation is added to the closure. A weakness of CWA is that already for very simple theories, like $A \vee B$ it is inconsistent. Since neither $\neg A$ nor $\neg B$ is derivable, we have to add them both which makes the whole set inconsistent.

GCWA is defi ned for positive disjunctive programs consisting of rules of the form

$$
A_{1} \vee \ldots \vee A_{n} \leftarrow B_{1}, \ldots, B_{m}
$$

by declaring all the minimal models to be the intended ones: 
Definition 6.2 (GCWA).

The generalized closed world assumption GCWA of $P$ is the semantics given by the set of all minimal Herbrand models of $P$ :

$$
\operatorname{GCWA}(P):=\operatorname{Min}-\operatorname{MOD}(P)
$$

Originally, Minker denoted by GCWA(P) a set of negated atoms with the property that $P \cup G C W A(P) \models \operatorname{not} A$ if and only if $\operatorname{MinMOD}(\mathrm{P}) \models \operatorname{not} A$ but we prefer here to denote by GCWA a semantics in the sense of Defin nition 2.4 on page 15.

GCWA is very important because it plays the same role for positive disjunctive programs as the least Herbrand model $M_{P}$ does for defi nite programs. In addition it turns out that some semantics SEM defi ned for arbitrary disjunctive programs (i. e. with default-negation) can be characterized, sometimes even implemented, by reducing them to positive programs and then applying recursively GCWA. Thus an appropriate procedure iterating GCWA can "implement" such semantics SEM.

Note also that as far as we consider deriving positive disjunctions, we stay entirely within classical logic - a positive disjunction is true in GCWA if and only if it follows from the program considered as a classical theory. Therefore this task can be accomplished be methods and techniques developed in theorem proving in the last 30 years. In fact this was one of the main starting points of the DisLoPproject in Koblenz (see Section 7.2).

Of course, GCWA is nothing else than Circumscription (see Section A.4) for a special class of theories. Methods developed for CIRC can be used to compute GCWA. For recent approaches that work in polynomial space see [Niemelä, 1996a; Niemelä, 1996b].

In Sections 2 and 3 we have introduced the general notion of a semantics and various principles. Do they carry over to the disjunctive case? Fortunately, the answer is yes. In addition, GCWA not only satisfi es all these properties, it is also uniquely characterized by them as the next theorem shows (we will introduce these properties in the next section).

Theorem 6.1 (Characterization of GCWA, [Brass and Dix, 1997]). Let SEM be a semantics satisfying GPPE and Elimination of Tautologies.

a) Then: $\operatorname{SEM}(P) \subseteq$ Min-MOD ${ }_{2-v a l}(P)$ for positive disj. programs $P$.

I.e. any such semantics is already based on 2-valued minimal models. In particular, GCWA is the weakest semantics with these properties.

b) If SEM is non-trivial and satisfi es in addition ${ }^{14}$ Isomorphy and Relevance, then it coincides with GCWA on positive disjunctive programs.

We end this section with the discussion of a well-known example that can not be handled adequately by Circumscription:

${ }^{14}$ See Section 7.1 for the precise definitions of Relevance and Isomorphy. 


\section{Example 6.1 (Poole's Broken Arm).}

Usually, a person's left arm is useable. But if the left arm is broken, it is an exception. The same statement holds for the right arm. Suppose that we saw Fred yesterday with a broken arm but we do not remember if it was the left or the right one. We also know that Fred can make out a cheque if he has at least one useable arm (he is ambidextrous) but that he is completely disabled if both arms are broken. Here is the natural formalization:

\begin{tabular}{|c|c|}
\hline left_use $(x)$ & $\leftarrow \operatorname{not} a b(l e f t, x)$ \\
\hline$a b(l e f t, x)$ & $\leftarrow \quad l e f t \_b r o k(x)$ \\
\hline right_use $(x)$ & $\leftarrow \operatorname{not} a b($ right,$x)$ \\
\hline$a b($ right,$x)$ & $\leftarrow \quad$ right_brok $(x)$ \\
\hline left_brok $($ Fred $) \vee$ right_brok $($ Fred $)$ & $\leftarrow$ \\
\hline make_cheque $(x)$ & $\leftarrow \quad$ left_use $(x)$ \\
\hline make_cheque $(x)$ & $\leftarrow \quad$ right_use $(x)$ \\
\hline $\operatorname{disabled}(x)$ & $\leftarrow \quad l e f t \_b r o k(x)$, right_brok $(x)$ \\
\hline
\end{tabular}

Of course, we expect that Fred is able to make out a cheque even without knowing which arm he is actually using. Also we derive that he is not (completely) disabled.

For general Circumscription, the problem is to rule out the unintended model where both arms are broken and Fred is disabled. As we will see later, both DWFS and DSTABLE derive that Fred is not disabled but only DSTABLE is strong enough to also conclude that Fred can make out a cheque.

\section{$6.2 D-W F S$}

Before we can state the defi nition of D-WFS we have to extend our principles to disjunctive programs with default-negation. We abbreviate general rules

$$
A_{1} \vee \ldots \vee A_{k} \leftarrow B_{1}, \ldots, B_{m}, \operatorname{not} C_{1}, \ldots, \operatorname{not} C_{n},
$$

by

$$
\mathcal{A} \leftarrow \mathcal{B}^{+}, \operatorname{not} \mathcal{B}^{-}
$$

where $\mathcal{A}:=\left\{A_{1}, \ldots, A_{k}\right\}, \mathcal{B}^{+}:=\left\{B_{1}, \ldots, B_{m}\right\}, \mathcal{B}^{-}:=\left\{C_{1}, \ldots, C_{n}\right\}$. We also generalize our notion of a semantics slightly:

Definition 6.3 (Operator $\sim$, Semantics $\mathcal{S}_{\sim}$ ).

By a semantic operator $\sim$ we mean a binary relation between logic programs and pure disjunctions which satisfi es the following three arguably obvious conditions:

1. Right Weakening: If $P \mid \sim \psi$ and $\psi \subseteq \psi^{\prime 15}$, then $P \sim \psi^{\prime}$.

2. Necessarily True: If $\mathcal{A} \leftarrow$ true $\in P$ for a disjunction $\mathcal{A}$, then $P \sim \mathcal{A}$.

\footnotetext{
${ }^{15}$ I. e. $\psi$ is a subdisjunction of $\psi^{\prime}$.
} 
3. Necessarily False: If $A \notin$ Head_atoms $(P)^{16}$ for some $\mathcal{L}$-ground atom $A$, then $P \sim \operatorname{not} A$.

Given such an operator $\sim$ and a logic program $P$, by the semantics $\mathcal{S}_{\sim}(P)$ of $P$ determined by $\mid \sim$ we mean the set of all pure disjunctions derivable by $\sim \sim$ from $P$, i.e., $\mathcal{S}_{\sim}(P):=\{\psi|P| \sim \psi\}$.

In order to give a unifi ed treatment in the sequel, we introduce the following notion:

Definition 6.4 (Invariance of $\sim$ under a Transformation).

Suppose that a program transformation Trans : $P \mapsto \operatorname{Trans}(P)$ mapping logic programs into logic programs is given. We say that the operator $\sim \sim$ is invariant under Trans (or that Trans is a $\sim$-equivalence transformation) iff

$$
P \mid \sim \psi \Longleftrightarrow \operatorname{Trans}(P) \sim \psi
$$

for any pure disjunction $\psi$ and any program $P$.

All our principles introduced below can now be naturally extended.

Definition 6.5 (Elimination of Tautologies, Non-Minimal Rules).

Semantics $\mathcal{S}_{\sim}$ satisfi es a) the Elimination of Tautologies, resp. b) the Elimination of Non-Minimal Rules iff $\sim$ is invariant under the following transformations:

a) Delete a rule $\mathcal{A} \leftarrow \mathcal{B}^{+} \wedge \operatorname{not} \mathcal{B}^{-}$with $\mathcal{A} \cap \mathcal{B}^{+} \neq \emptyset$.

b) Delete a rule $\mathcal{A} \leftarrow \mathcal{B}^{+} \wedge$ not $\mathcal{B}^{-}$if there is another rule $\mathcal{A}^{\prime} \leftarrow \mathcal{B}^{+^{\prime}} \wedge$ not $\mathcal{B}^{{ }^{\prime}}$ with $\mathcal{A}^{\prime} \subseteq \mathcal{A}, \mathcal{B}^{{ }^{\prime}} \subseteq \mathcal{B}^{+}$, and $\mathcal{B}^{-1} \subseteq \mathcal{B}^{-}$

Our partial evaluation principle has now to take into account disjunctive heads. The following defi nition was introduced independently by Sakama/Seki and Brass/Dix ([Brass and Dix, 1994; Brass and Dix, 1997; Sakama and Seki, 1994]):

Definition 6.6 (GPPE).

Semantics $\mathcal{S}_{\sim}$ satisfi es GPPE iff it is invariant under the following transformation: Replace a rule $\mathcal{A} \leftarrow \mathcal{B}^{+} \wedge$ not $\mathcal{B}^{-}$where $\mathcal{B}^{+}$contains a distinguished atom $B$ by the rules

$$
\mathcal{A} \cup\left(\mathcal{A}_{i} \backslash\{B\}\right) \leftarrow\left(\mathcal{B}^{+} \backslash\{B\}\right) \cup \mathcal{B}_{i}^{+} \wedge \operatorname{not}\left(\mathcal{B}^{-} \cup \mathcal{B}_{i}^{-}\right)(i=1, \ldots, n)
$$

where $\mathcal{A}_{i} \leftarrow \mathcal{B}_{i}^{+} \wedge$ not $\mathcal{B}_{i}^{-}(i=1, \ldots, n)$ are all the rules with $B \in \mathcal{A}_{i}$.

Note that we are free to select a specifi c positive occurrence of an atom $B$ and then perform the transformation. The new rules are obtained by replacing $B$ by the bodies of all rules $r$ with head literal $B$ and adding the remaining head atoms of $r$ to the head of the new rule.

\footnotetext{
${ }^{16}$ We denote by Head_atoms $_{(P)}$ the set of all (instantiations of) atoms ocurring in some rule-head of $P$.
} 
Here is the analogue of Principle 4 on page 22:

Definition 6.7 (Positive and Negative Reduction).

Semantics $\mathcal{S}_{\sim}$ satisfi es a) Positive, resp. b) Negative Reduction iff $\sim \sim$ is invariant under the following transformations:

a) Replace $\mathcal{A} \leftarrow \mathcal{B}^{+} \wedge \operatorname{not} \mathcal{B}^{-}$by $\mathcal{A} \leftarrow \mathcal{B}^{+} \wedge \operatorname{not}\left(\mathcal{B}^{-} \cap\right.$ Head_atoms $\left.(P)\right)$.

b) Delete $\mathcal{A} \leftarrow \mathcal{B}^{+} \wedge$ not $\mathcal{B}^{-}$if there is a rule $\mathcal{A}^{\prime} \leftarrow$ true with $\mathcal{A}^{\prime} \subseteq \mathcal{B}^{-}$.

Now the defi nition of a disjunctive counterpart of WFS is straightforward:

Definition 6.8 (D-WFS).

There exists the weakest semantics satisfying positive and negative Reduction, GPPE, Elimination of Tautologies and non-minimal Rules. We call this semantics D-WFS.

As it was the case for WFS, our calculus of transformations is also confluent ([Brass and Dix, 1998]).

Theorem 6.2 (Confluent Calculus for D-WFS).

The calculus consisting of our four transformations is confluent and terminating for propositional programs. I.e. we always arrive at an irreducible program, which is uniquely determined. The order of the transformations does not matter.

Therefore any program $P$ is associated a unique normalform $\operatorname{res}(P)$. The disjunctive wellfounded semantics of $P$ can be read off from $\operatorname{res}(P)$ as follows

$$
\begin{aligned}
\psi \in \mathrm{D}-\mathrm{WFS}(P) \Longleftrightarrow \quad & \text { there is } \mathcal{A} \subseteq \psi \text { with } \mathcal{A} \leftarrow \text { true } \in \text { res }(P) \text { or } \\
& \text { there is not } A \in \psi \text { and } A \notin \text { Head_atoms }(\text { res }(P)) .
\end{aligned}
$$

Note that the original defi nition of WFS, or any of its equivalent characterizations, does not carry over to disjunctive programs in a natural way.

Let us see how Example 6.1 on page 51 is handled by D-WFS. Applying GPPE and Reduction gives us the following residual program (we consider just the Fredinstantiations):

$$
\begin{array}{lll}
\text { left_use }(F) & \leftarrow \operatorname{not} a b(\text { left }, F) \\
\text { ab }(\text { left }, F) \vee \text { right_brok }(F) & \leftarrow & \\
\text { right_use }(F) & \leftarrow \operatorname{not} a b(\text { right }, F) \\
\text { ab }(\text { right, } F) \vee \text { left_brok }(F) & \leftarrow & \\
\text { left_brok }(F) \vee \text { right_brok }(F) & \leftarrow & \\
\text { make_cheque }(F) & \leftarrow \operatorname{not} a b(\text { left }, F) \\
\text { make_cheque }(F) & \leftarrow \operatorname{not} a b(\text { right }, F)
\end{array}
$$

Therefore we derive not disabled $(F)$, because it does not appear in any head of the residual program. All the remaining atoms are undefi ned.

Two properties of D-WFS are worth noticing 
- For positive disjunctive programs, D-WFS coincides with GCWA.

- For non-disjunctive programs with negation, D-WFS coincides with WFS.

\subsection{DSTABLE}

Unlike the wellfounded semantics, the original defi nition of stable models carries over to disjunctive programs quite easily:

\section{Definition 6.9 (DSTABLE).}

$N$ is called a stable model ${ }^{17}$ of $P$ iff $N \in \operatorname{Min}-\operatorname{Mod}\left(P^{N}\right)$.

In the last defi nition $P^{N}$ is the positive disjunctive program obtained from $P$ by applying the Gelfond/Lifschitz transformation (as introduced before Defi nition 3.4 on page 28-its generalization to disjunctive programs is obvious).

Analogously to D-WFS the following two properties of DSTABLE hold:

- For positive disjunctive programs, DSTABLE coincides with GCWA.

- For non-disjunctive programs with negation, DSTABLE coincides with STABLE.

What about our transformations introduced to defi ne D-WFS? Do they hold for DSTABLE? Yes, they are indeed true. The most diffi cult proof is the one for GPPE. It was proved in [Brass and Dix, 1999; Sakama and Seki, 1994] independently that stable models are preserved under GPPE. Moreover, Brass/Dix proved in [Brass and Dix, 1997] that STABLE can be almost uniquely determined by GPPE:

Theorem 6.3 (Characterization of DSTABLE).

Let SEM be a semantics satisfying GPPE, Elimination of Tautologies, and Elimination of Contradictions. Then: $\operatorname{SEM}(P) \subseteq \operatorname{STABLE}(P)$.

Moreover, DSTABLE is the weakest semantics satisfying these properties.

DSTABLE is stronger than D-WFS as can be seen from Example 6.1 on page 51. There we have exactly two stable models

1. left_use $(F)$, not $a b($ left, $F), a b($ right,$F)$, not right_use $(F)$, left_brok $(F)$, not right_brok $(F)$, make_cheque $(F)$, not disabled $(F)$,

2. right_use $(F), \operatorname{not} a b($ right,$F), a b($ left, $F)$, not left_use $(F)$, right_brok $(F)$, not left_brok $(F)$, make_cheque $(F)$, not disabled $(F)$.

In all of them, Fred is not disabled and can make out a cheque.

Of course, DSTABLE inherits the shortcomings of STABLE such as inconsistency and no goal-orientedness.

\footnotetext{
${ }^{17}$ Note that we only consider Herbrand models.
} 


\subsection{Other Semantics}

In this section we just want to mention some other disjunctive semantics proposed in the last years. First, there are semantics differing from GCWA in that they interpret " $\vee$ " inclusively, rather than exclusively (like GCWA does).

The corresponding semantics is called WGCWA (see [Rajasekar et al., 1989]) and is equivalent to the disjunctive database rule DDR considered in [Ross and Topor, 1988]. WGCWA has been considered as a more tractable (weaker) variant of GCWA (from the procedural point of view developed in [Rajasekar et al., 1989]).

\section{Example 6.2 (Inclusive versus Exclusive).}

$$
\begin{aligned}
P_{\text {incl/excl }}: & a \vee b \\
c & \leftarrow a, b
\end{aligned}
$$

Under an exclusive interpretation, not $c$ should be derivable. Indeed, we have $\operatorname{GCWA}\left(P_{\text {incl } / \text { excl }}\right)=\{\operatorname{not} c\}$.

Under an inclusive interpretation however, not $c$ should not be derivable. This is the case for WGCWA: $M_{P_{i n c l / e x c l}^{*}}=\{a, b, c\}$. The set of positive derivable literals is in both cases the same! If we replace the fi rst clause with $a$ or $b$, then not $c$ is derivable.

There are extensions of WGCWA to disjunctive programs with negation: [Sakama and Inoue, 1993; Ross, 1989; Dix, 1992b; Dix and Müller, 1994a].

There is also the book [Lobo et al., 1992] — the fi rst in-depth-study of disjunctive semantics with negation. However, we feel that these semantics have a drawback in that they are based on rather technical, complicated and not-easy-tounderstand fi xpoint defi nitions. These defi nitions leave a lot of room for modifi cations. But small modifi cations usually have a tremendous impact on the outcoming semantics. In addition these semantics do not allow for a proper treatment of defi nitional extensions (see Example 7.1 on page 60).

Let us discuss one more time the inclusivelexclusive meaning of $\vee$. Chiaki Sakama noted that inclusive vs. exclusive is not always fully determined by the underlying semantics. For example in the program $p \vee q, p \leftarrow q, q \leftarrow p$, GCWA derives both $p$ and $q$ so it is better to say that GCWA tends to interpret $\vee$ exclusively unless specifi ed otherwise. But weaker semantics cannot specify exclusive $\checkmark$. Therefore Sakama proposed in [Sakama, 1989] to consider programs with two different kinds of semantics: one for inclusive and one for exclusive behaviour. He defi ned a corresponding semantics, called possible model semantics PMS (see also [Sakama and Inoue, 1993; Sakama and Inoue, 1994]). Chan introduced this same idea under the name possible world semantics in [Chan, 1993]. PMS has the nice feature that it lies on the fi rst level of the polynomial hierarchy (see[Eiter and Gottlob, 1993a]).

Other approaches are due to Przymusinski: stationary-semantics $\mathcal{S T} \mathcal{N}$ and 


\begin{tabular}{|c|c|c|}
\hline \multirow[b]{3}{*}{ ( $P$ is positive) } & \multicolumn{2}{|c|}{ Complexity } \\
\hline & $\begin{array}{l}\text { 1. ord. prog. } \\
\text { (with functions) }\end{array}$ & $\begin{array}{c}\text { prop. prog. } \\
\text { (no variables) }\end{array}$ \\
\hline & $\begin{aligned} A: & \boldsymbol{\Sigma}_{1}^{\mathbf{0}} \text {-compl. } \\
\operatorname{not} A: & \boldsymbol{\Pi}_{2}^{\mathbf{0}} \text {-compl. }\end{aligned}$ & $\begin{aligned} A: & \text { co-NP-compl. } \\
\operatorname{not} A: & \boldsymbol{\Pi}_{2}^{\mathbf{P}} \text {-compl. }\end{aligned}$ \\
\hline $\begin{array}{c}\text { WGCWA } \\
(P \text { is positive })\end{array}$ & $\begin{aligned} A: & \boldsymbol{\Sigma}_{\mathbf{1}}^{\mathbf{0}} \text {-compl. } \\
\operatorname{not} A: & \boldsymbol{\Pi}_{\mathbf{1}}^{\mathbf{0}} \text {-compl. }\end{aligned}$ & $\begin{aligned} A: & \text { co-NP-compl. } \\
\operatorname{not} A: & \text { linear in }|P|\end{aligned}$ \\
\hline $\begin{array}{c}\text { PERFECT } \\
(P \text { is stratifi ed })\end{array}$ & arithm.-compl. & $\boldsymbol{\Pi}_{2}^{\mathbf{P}}$-compl. \\
\hline PMS & not yet studied & co-NP-compl \\
\hline $\begin{array}{l}\text { WPERFECT } \\
(P \text { is stratifi ed })\end{array}$ & arithm.-compl. & $\boldsymbol{\Pi}_{2}^{\mathbf{P}}$-compl. \\
\hline D-WFS & $\mathbf{\Pi}_{1}^{\mathbf{1}}$-compl. over $\mathbb{N}$ & $\mathbf{\Pi}_{2}^{\mathbf{P}}$-compl. \\
\hline DSTABLE & $\boldsymbol{\Pi}_{1}^{1}$-compl. over $\mathbb{N}$ & $\boldsymbol{\Pi}_{2}^{\mathbf{P}}$-compl. \\
\hline
\end{tabular}

Table 4. Complexity of Disjunctive Semantics

static-semantics STATIC (see [Przymusinski, 1995; Brass et al., 1999; Brass et al., 2001a]. STATIC is an improvement of his former stationary semantics that is very close to D-WFS: in fact it coincides with D-WFS if it is restricted to a common sublanguage ([Brass et al., 2001a]). This approach also allows us to consider a larger class of programs, namely those that contain not $\left(A_{1} \wedge \ldots \wedge A_{n}\right)$ in their bodies. Such programs are more expressible and therefore turn out to be even better suited for representation tasks.

Another approach differing from GCWA and WGCWA is considered in [Dix et al., 1994; Dix et al., 1996a; Bonatti, 1993].

\subsection{Complexity and Expressibility}

From the complexity point of view GCWA lies between CWA (which is $\Pi_{1}^{0}$ complete, see [Apt and Blair, 1990] and general Circumscription ( $\Sigma_{1}^{1}$-complete, see [Cadoli et al., 1992]): GCWA is $\Pi_{2}^{0}$-complete. For propositional programs we have to distinguish between deriving an atom or a literal. The fi rst problem is co-NP-complete while the second is even $\Pi_{2}^{P}$-complete (see [Imielinski, 1991]).

For deriving negated literals not $A$, WGCWA is $\Pi_{1}^{0}$-complete (like CWA) and therefore "better" than GCWA ( $\Pi_{2}^{0}$-complete). In the propositional case, WGCWA is polynomial while GCWA is $\Pi_{2}^{p}$-complete (both for the derivation of literals of the form $\operatorname{not} A$ ). 


\begin{tabular}{||c|c||}
\hline \hline & $\begin{array}{c}\text { Expressibility } \\
\text { 1. ord. prog. } \\
\text { (no functions })\end{array}$ \\
\hline $\begin{array}{c}\text { GCWA } \\
(P \text { is positive })\end{array}$ & $\subset \mathbf{\Pi}_{2}^{\mathbf{P}}$ \\
\hline $\begin{array}{c}\text { WGCWA } \\
(P \text { is positive })\end{array}$ & $\subset \mathbf{\Pi}_{\mathbf{2}}^{\mathbf{P}}$ \\
\hline $\begin{array}{c}\text { PERFECT } \\
(P \text { is stratifi ed })\end{array}$ & $=\mathbf{\Pi}_{\mathbf{2}}^{\mathbf{P}}$ \\
\hline $\begin{array}{c}\text { WPERFECT } \\
(P \text { is stratifi ed })\end{array}$ & $=\mathbf{\Pi}_{\mathbf{2}}^{\mathbf{P}}$ \\
\hline D-WFS & $=\mathbf{\Pi}_{2}^{\mathbf{P}}$ \\
\hline DSTABLE & $=\Pi_{2}^{\mathbf{P}}$ \\
\hline \hline
\end{tabular}

Table 5. Expressibility of Disjunctive Semantics

\section{WHAT DO WE WANT AND WHAT IS IMPLEMENTED?}

In this part we fi rst consider the question Is there an optimal semantics? (Section 7.1) and give in Section 7.2 an overview of all the existing implementations we are aware of. We also describe theoretical approaches that have not yet been implemented.

\subsection{What is the Best Semantics?}

Most probably there is no defi nite answer to the question in the title. Different knowledge representation tasks may ask for different semantics. Some might be better suited in special domains than others. What are reasonable properties that semantics should be checked against?

While many people defi ned in the last years new semantics by considering only few examples and appealing to their own personal intuitions they had about how these few examples should be handled, Dix tried to adjust and investigate abstract properties known in general nonmonotonic reasoning to semantics of logic programs ([Dix, 1991; Dix, 1992b; Dix, 1995a; Dix, 1995b]). He showed for example that WFS is cumulative and rational and that a semantics defi ned independently by Schlipf and Dix is the weakest extension of WFS satisfying Cut and Supraclassicality. Figure 3 on the following page illustrates the properties and the relationship between many semantics (note that $\leq_{k}$ refers to the knowledge ordering discussed 


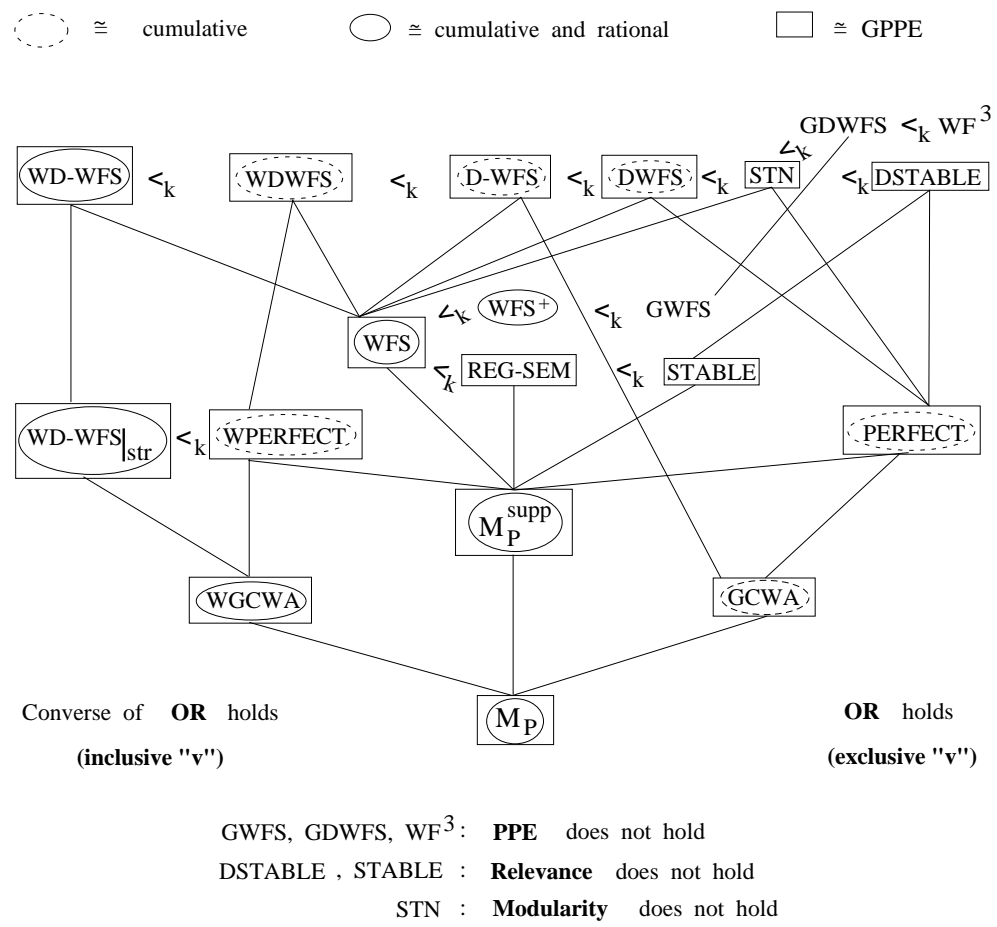

Figure 3. Semantics for Disjunctive Programs

in the beginning of Subsection 3.3: a semantics is strictly weaker, i.e. $\leq_{k}$, than another semantics if it derives strictly less literals). The distinction between inclusive and exclusive semantics is perhaps a bit misleading. We refer to the discussion in Subsection 6.4.

In Figure 4 on the next page normal programs are considered.

Besides such properties (which he calls strong) he defi ned also weak propertiesthese are conditions that any reasonable semantics should satisfy ([Dix, 1992a; Dix, 1995b]). The principles we have introduced in Sections 2, 3 belong to this sort. Let us take a closer look into some weak properties already mentioned (but not yet defi ned). We start with a property that is satisfi ed for any semantics we know:

Definition 7.1 (Isomorphy).

A semantics SEM satisfi es Isomorphy, iff

$$
\operatorname{SEM}(\mathcal{I}(P))=\mathcal{I}(\operatorname{SEM}(P))
$$

for all programs $P$ and isomorphisms $\mathcal{I}$ on the Herbrand base $B_{P}$. 


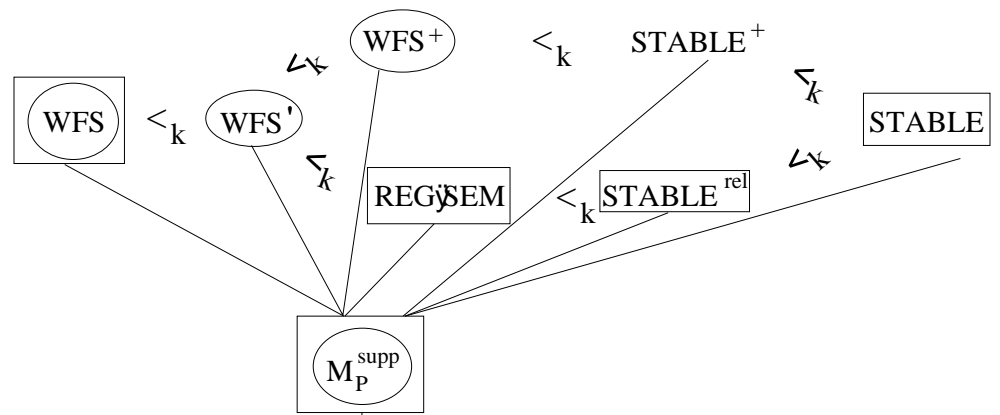

\section{$M_{P}$}

$\cong$ cumulative and rational

$\simeq \mathrm{GPPE}$

Figure 4. Semantics for Normal Programs

Isomorphy formalizes the intuition that a renaming of the program should have no influence on the semantics, as long as we also apply this same renaming to the semantics.

The next property gives a formal defi nition of the notion Goal-Orientedness. To state this conditions, we need the notion of the Dependency-Graph (Defi nition 3.2 on page 21) and the two defi nitions

- dependencies_of $(X):=\{A: X$ depends on $A\}$, and

- $\operatorname{rel} \_r u l(P, X)$ is the set of relevant rules of $P$ with respect to $X$, i.e. the set of rules that contain an $A \in$ dependencies_of $(X)$ in their head.

Given any semantics SEM and a program $P$, it is perfectly reasonable that the truthvalue of a literal $L$, with respect to $\operatorname{SEM}(\mathrm{P})$, only depends on the subprogram formed from the relevant rules of $P$ with respect to $L{ }^{18}$ This idea is formalized by:

Definition 7.2 (Relevance).

The principle of Relevance states: $L \in S E M(P)$ iff $L \in S E M($ rel_rul $(P, L))$.

\footnotetext{
${ }^{18}$ Let dependencies_of $(\operatorname{not} X):=$ dependencies_of $(X)$, and $\operatorname{rel} \_r u l(P, \operatorname{not} X):=$ rel_rul $(P, X)$.
} 
Note that the set of relevant rules of a program $P$ with respect to a literal $L$ contains all rules, that could ever contribute to $L$ 's derivation (or to its nonderivability). In general, $L$ depends on a large set of atoms: dependencies $\_f(L):=\{A: L$ depends on $A\}$. But rules that do not contain these atoms in their heads, will never contribute to their derivation or non-derivation. Therefore, these rules should not affect the meaning of $L$ in $P$. STABLE does not satisfy this principle. This is due to the nonexistence of stable models by adding a clause " $c \leftarrow \operatorname{not} c$ " to a program.

We have already introduced GPPE above. It is an extension of the following property for non-disjunctive programs:

\section{Definition 7.3 (PPE).}

Let $P$ be an instantiated program and let the atom $c$ occur positively in $P$. Let $c \leftarrow r h s_{1}, \ldots, c \leftarrow r h s_{n}$ be all the rules of $P$ with $c$ in their heads.

Any program clause of the form "head $\leftarrow c$, body" can be replaced by the rules

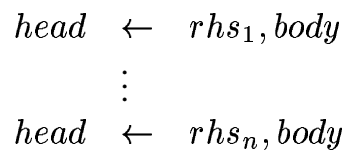

Note that the rules $c \leftarrow r h s_{1}, \ldots c \leftarrow r h s_{n}$ are not removed (in contrast to the weak version of PPE). We call the program obtained in this way $P^{\prime}$.

The principle of partial evaluation is: $S E M\left(P^{\prime}\right)=S E M(P)$.

GPPE is obtained from PPE by weakening the assumption that $c$ only occurs positively. We note that most semantics defi ned by Minker and his group do not satisfy this condition:

Example 7.1 (Extension-by-Definition, [Dix, 1991]).

We consider the following two programs:

$$
\begin{array}{rlrl}
P_{G W F S}: & p \leftarrow \operatorname{not} b & P_{G W F S c}: & p \leftarrow \operatorname{not} b \\
a & \leftarrow \operatorname{not} b & & a \leftarrow \operatorname{not} b \\
b & \leftarrow c & b \leftarrow p, \operatorname{not} a \\
c & \leftarrow p, \operatorname{not} a & c & \leftarrow \operatorname{not} a
\end{array}
$$

$\operatorname{GWFS}\left(P_{G W F S}\right)$ entails not $c$, because $\operatorname{Min}-\operatorname{MOD}\left(P_{G W F S}\right)=\{\{p, a\},\{b\}\}$ and thus also (by simple negation-as-failure reasoning) not $b, p$ and $a$. Also Min$\operatorname{MOD}\left(P_{G W F S_{c}}\right)=\{\{p, a\},\{b\}\}$ but negation-as-failure can not be applied like before. Therefore GWFS $\left(P_{G W F S_{c}}\right)$ does not entail not $b$, nor $p$ nor $a$.

$P_{G W F S c}$ partial evaluates $P_{G W F S}$ : the last but one clause was transformed into another one by expanding the defi nition of $c$. Obviously, a semantics should assign the same meaning to these programs: unfortunately GWFS does not!

The next principle, Modularity, has some similarities with PPE. It enables us to compute a semantics by modularizing it into certain "subprograms" (formed of 
the relevant rules). The semantics of these modules can be computed fi rst and the semantics of the whole program can be determined by reducing this program with literals that were already determined.

\section{Definition 7.4 (Modularity).}

Let $P=P_{1} \cup P_{2}$ and for every $A \in B_{P_{2}}: \operatorname{rel} \_r u l(P, A) \subseteq P_{2}$.

The principle of Modularity is: $\operatorname{SEM}(P)=\operatorname{SEM}\left(P_{1} \operatorname{SEM}\left(P_{2}\right) \cup P_{2}\right)$.

To illustrate this property, we compare the program

$$
\begin{aligned}
P_{1}: & b \quad \leftarrow y \\
& y \vee x \\
z \vee y & \\
m & \leftarrow x, z, b \\
y & \leftarrow \operatorname{not} m
\end{aligned}
$$

with the union of the following two programs

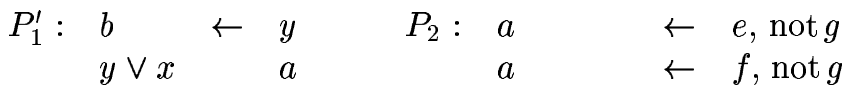

$$
\begin{aligned}
& z \vee y \quad a \quad a \quad \leftarrow f \text {, note } \\
& m \leftarrow x, z, b \quad a \quad \leftarrow g, \operatorname{not} e \\
& y \leftarrow \operatorname{not} m \quad e \vee f \vee g
\end{aligned}
$$

$P_{2}$ is a stratifi ed program and $\mathcal{S T N}$ derives $a$. Concerning $P_{1}$, different intuitions seem possible. One can argue, that not $m$ should be derivable, since the only way to derive $m$ is by using the fourth clause, which means deriving $b$, which means deriving $y$ which excludes deriving $x$ or $z$. This is the way, $P_{1}$ is handled by the fi rst version of $\mathcal{S} \mathcal{T} \mathcal{N}$. The second (fi nal) version $\mathcal{S T} \mathcal{N}$ does not derive not $m$. But if we apply $\mathcal{S} \mathcal{T} \mathcal{N}$ to $P_{1}^{\prime} \cup P_{2}$, then not $m$ is derivable. This shows that weak Modularity is not satisfi ed: we consider this to be a serious shortcoming.

Typical results of Dix are

- WFS is the weakest semantics satisfying some of these weak properties,

- WFS can be uniquely characterized if some strong properties are added.

We conclude with Table 6 on the next page: an overview of the properties of some semantics mentioned above.

The bad properties of the PMS (failure of Relevance) stem from the fact that it was originally based on stable models. But the underlying idea of PMS is to transform disjunctive programs into non-disjunctive ones and then applying a semantics for non-disjunctive programs. By choosing semantics different from STABLE, PMS inherits other properties (see [Sakama and Inoue, 1994]).

\subsection{Query-Answering Systems and Implementations}

In this section we give a rough overview of what semantics have been implemented so far and where they are available. As already explained in Sections 3.5, 6.5, 


\begin{tabular}{|ll|l|l|c|c|c|c|}
\hline \multicolumn{7}{|c|}{ Properties of Logic-Programming Semantics } \\
\hline \hline Semantics Reference & Domain & Taut. & GPPE & Red. & NMin. & Rel. \\
\hline comp & Cla78 & Nondis. & - & $\bullet$ & $\bullet$ & $\bullet$ & - \\
GCWA & Min82 & Pos. & $\bullet$ & $\bullet$ & $\bullet$ & $\bullet$ & $\bullet$ \\
WGCWA RosTop88 & Pos. & - & $\bullet$ & $\bullet$ & - & $\bullet$ \\
DSTABLEGelLif91 & Dis. & $\bullet$ & $\bullet$ & $\bullet$ & $\bullet$ & - \\
WFS & vGeld. etal88 & Nondis. & $\bullet$ & $\bullet$ & $\bullet$ & $\bullet$ & $\bullet$ \\
ST N & Prz91 & Dis. & $\bullet$ & $\bullet$ & $\bullet$ & $\bullet$ & $\bullet$ \\
STATIC & Prz95 & Dis. & $\bullet$ & $\bullet$ & $\bullet$ & $\bullet$ & $\bullet$ \\
D-WFS & BraDix95 & Dis. & $\bullet$ & $\bullet$ & $\bullet$ & $\bullet$ & $\bullet$ \\
DWFS & Dix92 & Dis. & $\bullet$ & $\bullet$ & $\bullet$ & $\bullet$ & $\bullet$ \\
Str. WFS & Ros92 & Dis. & - & - & $\bullet$ & - & $\bullet$ \\
WD-WFS BraDix95 & Dis. & - & $\bullet$ & $\bullet$ & - & $\bullet$ \\
WDWFS & Dix92 & Dis. & - & $\bullet$ & $\bullet$ & - & $\bullet$ \\
PMS & SakIno94 & Dis. & - & - & $\bullet$ & - & - \\
\hline
\end{tabular}

Table 6. Semantics and Their Equivalence-Transformations

our NMR-semantics are undecidable in general. Nevertheless we think it is very important to have running systems that

1. can handle programs with free variables, and

2. are Goal-Oriented.

To ensure completeness (or termination) we need then additional requirements like allowedness (to prevent floundering, see Section 3.1) and no function symbols.

Although these restrictions ensure the Herbrand-universe to be fi nite (and thus we are really considering a propositional theory) we think that such a system has great advantages over a system that can just handle ground programs. For a language $\mathcal{L}$, the fully instantiated program can be quite large and diffi cult to handle effectively. The goal-orientedness (or Relevance as introduced in Section 7.1) is also important-after all this was one reason of the success of SLD-Resolution. As noted above, such a goal-oriented approach is not possible for STABLE.

\section{LP-Semantics}

Various commercial PROLOG-systems perform variants of SLDNF-Resolution. Chan's constructive negation has also been implemented as part of the mastertheses [Ludäscher, 1991; Vorbeck, 1991].

Currently, a library of implemented logic programming systems and interesting test-cases for such systems is collected as a project of the artifi cial intelligence group at Koblenz. We refer to http://www . uni-koblenz.de/ag-ki/LP/>. 
Non-Disjunctive NMR-Semantics

There are many theoretical papers that deal with the problem of implementation ([Bol and Degerstedt, 1993; Kemp et al., 1991; Degerstedt and Nilsson, 1995; Fernández et al., 1993]) but only few running systems. The problem of handling and representing ground programs given a non-ground one has also been adressed [Kagan et al., 1994; Kagan et al., 1995; Eiter et al., 1997a].

In [Bell et al., 1993; Bell et al., 1994] the authors showed how the problem of computing stable models can be transformed to an Integer-Linear Programming Problem. This has been extended in [Dix and Müller, 1993] to disjunctive programs.

Inoue et. al. show in [Inoue et al., 1992] how to compute stable models by transforming programs into propositional theories and then using a model-generation theorem prover.

In Berne, Switzerland, a group around G. Jäger has built a non-monotonic reasoning system which incorporates various monotonic and non-monotonic logics. We refer to http://lwbwww . unibe.ch:8080/LWBinfo.html.

Extended logic programs under the well-founded semantics are considered by Pereira and his colleagues: [Pereira et al., 1993; Alferes and Pereira, 1996]. The REVISE system, which deals with contradiction removal for paraconsistent programs in this semantics, can be found in <http: //www . uni-koblenz . de/ag-ki/ $\mathrm{LP} />$ too.

In [Niemelä and Simons, 1996], an implementation of STABLE with a special eye on complexity is described. The resulting system, smodels, is publicly available (see <http: //www.tcs.hut.fi/Software/smodels/>) and seems to outperform most other approaches to implementing STABLE. More references can be found in [Dix et al., 2001a]. Many problems in model checking, planning, diagnosis and various other areas can be translated into logic programs in such a way, that stable models of these programs correspond exactly to solutions of the original problems.

The most advanced system, XSB, has been implemented by David Warren and his group in Stony Brook based on OLDT-algorithm of [Tamaki and Sato, 1986]. They fi rst developed a meta-interpreter (SLG, see [Chen and Warren, 1996]) in PROLOG and then directly modifi ed the WAM for a direct implementation of WFS (XSB). They use tabling-methods and a mixture of Top-Down and bottomup evaluation to detect loops. The XSB system is complete and terminating for non-floundering DATALOG. It also works for general programs but termination is not guaranteed. This system is described in [Chen and Warren, 1993; Chen et al., 1995; Chen and Warren, 1995; Swift, 1999], and is available by anonymous ftp from ftp.cs.sunysb.edu/pub/XSB/. We also refer to [Apt et al., 1999]. 


\section{Disjunctive NMR-Semantics}

There are theoretical descriptions of implementations that have not yet been implemented: [Fernández and Minker, 1995; Minker and Ruiz, 1995; Costantini and Lanzarone, 1995]. Also Sakama and Seki describe an approach for fi rst-order disjunctive programs ([Sakama and Seki, 1997]).

Here are some implemented systems. Inoue et. al. show in [Inoue et al., 1992] how to compute stable models for extended disjunctive programs in a bottom-upfashion using a theorem prover.

The approach of Bell et. al. ([Nerode et al., 1991]) was used by Dix/Müller to implement versions of the stationary semantics of Przymusinski ([Przymusinski, 1991]): [Müller and Dix, 1993; Dix and Müller, 1992].

Brass/Dix have implemented both D-WFS and DSTABLE for allowed DATALOG programs ([Brass and Dix, 1995]). An implementation of static semantics is described in [Brass et al., 1999].

Seipel has implemented in his DisLog-system various (modifi ed versions of) semantics of Minker and his group (http: / / sunwww . informatik . uni-tuebingen . de:8080/dislog/dislog.tar.Z).

The DisLoP project (1995-2000, see [Aravindan et al., 1997]) aimed at extending certain theorem proving concepts, such as restart model elimination and hyper tableaux calculi, for disjunctive logic programming. This system can be extended to handle non-monotonic semantics such as D-WFS, STATIC etc. In particular, an implementation of D-WFS for general disjunctive programs which works in polynomial space is available ([Brass et al., 2001a]). An extension to fi rst-order programs is proposed in [Dix and Stolzenburg, 1998]. Information on the DisLoP project and related publications can be obtained from the WWW page

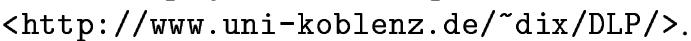

The most advanced system however is dlv ([Eiter et al., 1997b; Eiter et al., 1998]). It constitutes a knowledge representation system, based on disjunctive logic programming, which offers front-ends to several advanced KR formalisms (developed since the end of 1996). Major emphasis has been put on advanced knowledge modelling features. The kernel language, which extends disjunctive logic programming by true negation and integrity constraints, allows for representing complex knowledge based problems in a highly declarative fashion [Eiter et al., 1998]. The system runs in polynomial space and single exponential time, and is able to effi ciently recognize and process syntactical subclasses of disjunctive logic programs which have lower computational complexity than the general case (like, e.g., programs with head-cycle free disjunction or stratifi ed negation).

An important outcome of the Dagstuhl Seminar 9627 ([Dix et al., 1996b]) was to construct a web page to collect and disseminate information on various logic programming systems that concentrate on non-monotonic aspects (different kinds of negation, disjunction, abduction etc.). This web page is actively maintained at the URL <http://www.uni-koblenz.de/ag-ki/LP/>. In addition the Logic Programming and Nonmonotonic Reasoning-conference 1997 ([Dix et 
al., 1997a]) contained for the first time a special track on implementations and working systems. Various LP-systems were also demonstrated at NMR 2000 $\langle$ http://www.cs . engr .uky. edu/nmr2000〉.

\section{OUTLOOK}

Currently, a new logic programming paradigm seems to emerge: answer set programming. The idea is to take the credulous viewpoint under the stable semantics seriously: the set of all stable models of a program really is the main object described by this program. Viewed in this way, even propositional programs can encode second order objects. Thus when a problem is translated into a logic program under the stable semantics, the program itself can be seen as a compact encoding of the set of solutions of the original problem. We refer to [Dix, 1998; Apt et al., 1999; Niemelä, 1999].

Recently, methods of logic programming have also been successfully applied in the area of multi agent systems [Eiter et al., 2000; Eiter et al., 1999; Dix et al., 2000b; Dix et al., 2000a; Dix et al., 2001b; Subrahmanian et al., 2000; Dix, 2001].

\section{ACKNOWLEDGEMENTS}

We thank Chandrabose Aravindan, Norbert E. Fuchs, Ulrich Furbach, Ilkka Niemelä and Chiaki Sakama who provided us with many useful remarks. Katrin Erk and Dorothea Schäfer proofread parts of the paper-special thanks to them.

\section{BIBLIOGRAPHY}

[Alferes and Pereira, 1995] Jose Julio Alferes and Luiz Moniz Pereira. An argumentation theoretic semantics based on non-refutable falsity. In J. Dix, L. Pereira, and T. Przymusinski, editors, Nonmonotonic Extensions of Logic Programming, LNAI 927, pages 3-22. Springer, Berlin, 1995.

[Alferes and Pereira, 1996] Jose Julio Alferes and Luiz Moniz Pereira, editors. Reasoning with Logic Programming, LNAI 1111, Berlin, 1996. Springer.

[Alferes et al., 1996] Jose Julio Alferes, Luiz Moniz Pereira, and Teodor Przymusinski. Strong and Explicit Negation in Non-Monotonic Reasoning and Logic Programming. In J.J Alferes, L.M. Pereira, and E. Orlowska, editors, Logics in Artificial Intelligence (JELIA '96), LNCS 1126, pages 143-163. Springer, 1996.

[Apt and Blair, 1990] Krzysztof R. Apt and Howard A. Blair. Arithmetic Classification of perfect Models of stratified Programs. Fundamenta Informaticae, XIII:1-17, 1990. Addendum in Vol. XIV, pages 339-344, 1991.

[Apt and Bol, 1994] Krzysztof R. Apt and Roland N. Bol. Logic Programming and Negation: A Survey. Journal of Logic Programming, 19-20:9-71, 1994.

[Apt et al., 1999] K. R. Apt, V. Marek, M. Truszczynski, and D. S. Warren, editors. The Logic Programming Paradigm: Current Trends and Future Directions, Berlin, 1999. Springer.

[Apt, 1990] Krzysztof R. Apt. Logic programming. In J. van Leeuwen, editor, Handbook of Theoretical Computer Science, Vol. B, chapter 10, pages 493-574. Elsevier Science Publishers, 1990.

[Aravindan et al., 1997] Chandrabose Aravindan, Jürgen Dix, and Ilkka Niemel“̈. Dislop: A research project on disjunctive logic programming. AI Communications, 10(3/4):151-165, 1997. 
[Baader and Hollunder, 1995] Franz Baader and Bernd Hollunder. Priorities on defaults with prerequisite and their application in treating specificity in terminological default logic. Journal of Automated Reasoning, 15:41-68, 1995.

[Balc'azar et al., 1988 J.L. Balc'azar, I. Dóaz, and J. Gabarr'o. Structural Complexity I. SpringerVerlag, Berlin, 1988.

[Baral and Gelfond, 1994] Chitta Baral and Michael Gelfond. Logic Programming and Knowlege Representation. Journal of Logic Programming, 19-20:73-148, 1994.

[Baral and Subrahmanian, 1991] Chitta Baral and V.S. Subrahmanian. Dualities between Alternative Semantics for Logic Programming and Non-monotonic Reasoning. In Anil Nerode, Wiktor Marek, and V. S. Subrahmanian, editors, Logic Programming and Non-Monotonic Reasoning, Proceedings of the first International Workshop, pages 69-86, Cambridge, Mass., July 1991. Washington D.C, MIT Press.

[Baral and Subrahmanian, 1992] Chitta Baral and V.S. Subrahmanian. Stable and Extension Class Theory for Logic Programs and Default Logics. Journal of Automated Reasoning, 8, No. 3:345366, 1992.

[Barbuti and Martelli, 1986] R. Barbuti and M. Martelli. Negation as Failure. Completeness of the Query Evaluation Process for Horn Clause Programs with Recursive Definition. Journal of Automated Reasoning, 2:155-170, 1986.

[Barwise, 1975] Jon Barwise. Admissible Sets and Structures. Springer, 1975.

[Beeri and Ramakrishnan, 1991] Catril Beeri and Raghu Ramakrishnan. On the power of magic. The Journal of Logic Programming, 10:255-299, 1991.

[Bell et al., 1993] Colin Bell, Anil Nerode, Raymond T. Ng, and V. S. Subrahmanian. Implementing Stable Semantics by Linear Programming. In Luis Moniz Pereira and Anil Nerode, editors, Logic Programming and Non-Monotonic Reasoning, Proceedings of the Second International Workshop, pages 23-42, Cambridge, Mass., July 1993. Lisbon, MIT Press.

[Bell et al., 1994] Colin Bell, Anil Nerode, Raymond T. Ng, and V. S. Subrahmanian. Mixed Integer Programming Methods for Computing Non-Monotonic Deductive Databases. Journal of the ACM, 41(6):1178-1215, November 1994.

[Ben-Eliyahu and Dechter, 1992] Rachel Ben-Eliyahu and Rina Dechter. Propositional Semantics for Disjunctive Logic Programs. In K. R. Apt, editor, LOGIC PROGRAMMING: Proceedings of the 1992 Joint International Conference and Symposium, pages 813-, Cambridge, Mass., November 1992. MIT Press.

[Benferhat et al., 1993] Salem Benferhat, Claudette Cayrol, Didier Dubois, Jerome Lang, and Henri Prade. Inconsistency management and prioritized syntax-based entailment. In Proc. 13th International Joint Conference on Artificial Intelligence, pages 640-645. Morgan Kaufman, 1993.

[Bidoit and Froidevaux, 1991a] Nicole Bidoit and Christine Froidevaux. General logical Databases and Programs: Default Logic Semantics and Stratification. Information and Computation, 91:1554, 1991.

[Bidoit and Froidevaux, 1991b] Nicole Bidoit and Christine Froidevaux. Negation by Default and unstratifiable logic Programs. Theoretical Computer Science, 78:85-112, 1991.

[Bol and Degerstedt, 1993] Roland N. Bol and L. Degerstedt. Tabulated resolution for well-founded semantics. In Proc. Int. Logic Programming Symposium'93, Cambridge, Mass., 1993. MIT Press.

[Bonatti, 1993] Piero Bonatti. Shift-based semantics: general results and applications. Technical Report CD-TR-93-59, Technical University of Vienna, Inst. f ur Informationssysteme, 1993.

[B"orger, 1987 Egon B orger. Unsolvable Decision Problems For Prolog Programs. In Egon B orger, editor, Computation Theory and Logic, LNCS 270, pages 37-47, Berlin, 1987. Springer

[Brass and Dix, 1994] Stefan Brass and J urgen Dix. A disjunctive semantics based on unfolding and bottom-up evaluation. In Bernd Wolfinger, editor, Innovationen bei Rechen- und Kommunikationssystemen, (IFIP '94-Congress, Workshop FG2: Disjunctive Logic Programming and Disjunctive Databases), pages 83-91, Berlin, 1994. Springer.

[Brass and Dix, 1995] Stefan Brass and Jürgen Dix. A General Approach to Bottom-Up Computation of Disjunctive Semantics. In J. Dix, L. Pereira, and T. Przymusinski, editors, Nonmonotonic Extensions of Logic Programming, LNAI 927, pages 127-155. Springer, Berlin, 1995.

[Brass and Dix, 1997] Stefan Brass and Jürgen Dix. Characterizations of the Disjunctive Stable Semantics by Partial Evaluation. Journal of Logic Programming, 32(3):207-228, 1997.

[Brass and Dix, 1998] Stefan Brass and J urgen Dix. Characterizations of the Disjunctive Wellfounded Semantics: Conflient Calculi and Iterated GCWA. Journal of Automated Reasoning, 20(1):143-165, 1998. 
[Brass and Dix, 1999] Stefan Brass and Jüurgen Dix. Semantics of (Disjunctive) Logic Programs Based on Partial Evaluation. Journal of Logic Programming, 38(3):167-213, 1999.

[Brass et al., 1999] Stefan Brass, Jüurgen Dix, and Teodor Przymusinski. Computation of the semantics of autoepistemic belief theories. Artificial Intelligence, 112(1-2):104-123, 1999. (Partial results appeared in: Super Logic Programs. Proceedings of the 5-th International Conference on Principles of Knowledge Representation and Reasoning. Boston, MA, pages 529-541, 1996. Morgan Kaufman.).

[Brass et al., 2001a] Stefan Brass, J ürgen Dix, Ilkka Niemel"a, and Teodor Przymusinski. On the Equivalence of the Static and Disjunctive Well-Founded Semantics and its Computation. Theoretical Computer Science, 258(1-2):523-553, 2001.

[Brass et al., 2001b] Stefan Brass, Jüurgen Dix Burkhardt Freitag, and Ulrich Zukowski. Transformation-based bottom-up computation of the well-founded model. Theory and Practice of Logic Programming, to appear, 2001.

[Brewka and Eiter, 1999] Gerhard Brewka and Thomas Eiter. Preferred answer sets. Artificial Intelligence Journal, 109:297-356, 1999.

[Brewka et al., 1997] Gerd Brewka, J ürgen Dix, and Kurt Konolige. Nonmonotonic Reasoning: An Overview. CSLI Lecture Notes 73. CSLI Publications, Stanford, CA, 1997.

[Brewka, 1994] G. Brewka. Adding priorities and specificity to default logic. In Logics in Artificial Intelligence, Proc. JELIA-94, York. Springer, 1994.

[Brewka, 1996a] Gerd Brewka. Principles of Knowledge Representation. CSLI. Dikran, 1996.

[Brewka, 1996b] Gerhard Brewka. Well-founded semantics for extended logic programs with dynamic preferences. Journal of Artificial Intelligence Research, 4:19-36, 1996.

[Brewka, 2001] Gerhard Brewka. On the relation between defeasible logic and well-founded semantics. In Proc. LPNMR 2001. Springer, 2001.

[Bry, 1990] Francois Bry. Query evaluation in recursive databases: bottom-up and top-down reconciled. Data \& Knowledge Engineering, 5:289-312, 1990.

[Cadoli and Schaerf, 1993] Marco Cadoli and Marco Schaerf. A Survey of Complexity Results for Non-Monotonic Logics. Journal of Logic Programming, 17:127-160, 1993.

[Cadoli et al., 1992] Marco Cadoli, Thomas Eiter, and Georg Gottlob. An efficient method for eliminating varying predicates from a circumscription. Artificial Intelligence Journal, 54:397-410, 1992.

[Cadoli et al., 1995] M. Cadoli, F. M. Donini, P. Liberatore, and M. Schaerf. The size of a revised knowledge base. In PODS '95, pages 151-162, 1995.

[Cadoli et al., 1996] Marco Cadoli, Francesco M. Donini, and Marco Schaerf. Is intractability of non-monotonic reasoning a real drawback? Artificial Intelligence Journal, 88:215-251, 1996.

[Cadoli et al., 1997] Marco Cadoli, Francesco M. Donini, Marco Schaerf, and Riccardo Silvestri. On compact representations of propositional circumscription. Theoretical Computer Science, 182:183$202,1997$.

[Cavedon and Lloyd, 1989] L. Cavedon and J.W. Lloyd. A Completeness Theorem for SLDNFResolution. Journal of Logic Programming, 7:177-191, 1989.

[Chan and Wallace, 1989] David Chan and Mark Wallace. An Experiment with programming using pure Negation. Technical Report TR, ECRC, July 1989.

[Chan, 1988] David Chan. Constructive negation based on the completed database. In Proc. 1988 Conf. and Symp. on Logic Programming, pages 111-125, September 1988.

[Chan, 1993] Edward P.F. Chan. A Possible World Semantics for Disjunctive Databases. IEEE Trans. on Knowledge and Data Engineering, 5(2):282-292, 1993.

[Chen and Warren, 1993] Weidong Chen and David S. Warren. A Goal Oriented Approach to Computing The Well-founded Semantics. Journal of Logic Programming, 17:279-300, 1993.

[Chen and Warren, 1995] Weidong Chen and David S. Warren. Computing of Stable Models and its Integration with Logical Query Processing. IEEE Transactions on Knowledge and Data Engineering, 17:279-300, 1995.

[Chen and Warren, 1996] Weidong Chen and David S. Warren. Tabled Evaluation with Delaying for General Logic Programs. Journal of the ACM, 43(1):20-74, January 1996.

[Chen et al., 1995] Weidong Chen, Terrance Swift, and David S. Warren. Efficient Top-Down Computation of Queries under the Well-Founded Semantics. Journal of Logic Programming, 24(3):219$245,1995$.

[Chomicki and Subrahmanian, 1990] Jan Chomicki and V.S. Subrahmanian. Generalized Closed World Assumption is $\Pi_{2}^{0}$-Complete. Information Processing Letters, 34:289-291, 1990. 
[Clark, 1978] Keith L. Clark. Negation as Failure. In H. Gallaire and J. Minker, editors, Logic and Data-Bases, pages 293-322. Plenum, New York, 1978.

[Colmerauer et al., 1973] A. Colmerauer, H. Kanoui, R. Pasero, and P. Roussel. Un système de communication homme-machine en français. Technical report, Groupe de Intelligence Artificielle Universite de Aix-Marseille II, 1973.

[Costantini and Lanzarone, 1995] Stefania Costantini and Gaetano A. Lanzarone. Static Semantics as Program Transformation and Well-founded Computation. In J. Dix, L. Pereira, and T. Przymusinski, editors, Nonmonotonic Extensions of Logic Programming, LNAI 927, pages 156-180. Springer, Berlin, 1995.

[Decker and Cavedon, 1990] Hendrik Decker and Lawrence Cavedon. Generalizing syntactic properties which ensure that SLDNF-Resolution is complete and fbunder-free. Technical report, ECRC Munich, January 1990.

[Degerstedt and Nilsson, 1995] Lars Degerstedt and Ulf Nilsson. Magic Computation of Wellfounded Semantics. In J. Dix, L. Pereira, and T. Przymusinski, editors, Nonmonotonic Extensions of Logic Programming, LNAI 927, pages 181-204. Springer, Berlin, 1995.

[Dix and M"üler, 1992 J ürgen Dix and Martin M"üler. Abstract Properties and Computational Complexity of Semantics for Disjunctive Logic Programs. In Proc. of the Workshop W1, Structural Complexity and Recursion-theoretic Methods in Logic Programming, following the JICSLP '92, pages 15-28. H. Blair and W. Marek and A. Nerode and J. Remmel, November 1992. also available as Technical Report 13/93, University of Koblenz, Department of Computer Science.

[Dix and Müuller, 1993 Jüurgen Dix and Martin M üller. Implementing Semantics for Disjunctive Logic Programs Using Fringes and Abstract Properties. In Luis Moniz Pereira and Anil Nerode, editors, Logic Programming and Non-Monotonic Reasoning, Proceedings of the Second International Workshop, pages 43-59, Cambridge, Mass., July 1993. Lisbon, MIT Press.

[Dix and M"uller, 1994a Jüurgen Dix and Martin M̈uller. An Axiomatic Framework for Representing and Characterizing Semantics of Disjunctive Logic Programs. In Pascal Van Hentenryck, editor, Proceedings of the 11th Int. Conf. on Logic Programming, S. Margherita Ligure, pages 303-322. MIT, June 1994.

[Dix and Müller, 1994b Jürgen Dix and Martin Müller. Partial Evaluation and Relevance for Approximations of the Stable Semantics. In Z.W. Ras and M. Zemankova, editors, Proceedings of the 8th Int. Symp. on Methodologies for Intelligent Systems, Charlotte, NC, 1994, LNAI 869, pages 511-520, Berlin, 1994. Springer.

[Dix and Müller, 1994d J urgen Dix and Martin Müller. The Stable Semantics and its Variants: A Comparison of Recent Approaches. In L. Dreschler-Fischer and B. Nebel, editors, Proceedings of the 18th German Annual Conference on Artificial Intelligence (KI '94), Saarbrücken, Germany, LNAI 861, pages 82-93, Berlin, 1994. Springer.

[Dix and Stolzenburg, 1998] J'ürgen Dix and Frieder Stolzenburg. A Framework to incorporate Nonmonotonic Reasoning into Constraint Logic Programming. Journal of Logic Programming, 37(1,2,3):47-76, 1998. Special Issue on Constraint Logic Programming, Guest Editors: Kim Marriott and Peter Stuckey.

[Dix et al., 1994] J ürgen Dix, Georg Gottlob, and Viktor Marek. Causal Models for Disjunctive Logic Programs. In Pascal Van Hentenryck, editor, Proceedings of the 11th Int. Conf. on Logic Programming, S. Margherita Ligure, pages 290-302. MIT, June 1994.

[Dix et al., 1995] J. Dix, L. Pereira, and T. Przymusinski, editors. Non-Monotonic Extensions of Logic Programming, LNAI 927, Berlin, 1995. Springer.

[Dix et al., 1996a] J'urgen Dix, Georg Gottlob, and Viktor Marek. Reducing disjunctive to nondisjunctive semantics by shift-operations. Fundamenta Informaticae, XXVIII(1/2):87-100, 1996.

[Dix et al., 1996b] J'ürgen Dix, Donald Loveland, Jack Minker, and David. S. Warren. Disjunctive Logic Programming and databases: Nonmonotonic Aspects. Technical Report Dagstuhl Seminar Report 150, IBFI GmbH, Schloß Dagstuhl, 1996.

[Dix et al., 1997a] J. Dix, U. Furbach, and A. Nerode, editors. Logic Programming and Nonmonotonic Reasoning, LNAI 1265, Berlin, 1997. Springer.

[Dix et al., 1997b] J. Dix, L. Pereira, and T. Przymusinski, editors. Non-Monotonic Extensions of Logic Programming, LNAI 1216, Berlin, 1997. Springer.

[Dix et al., 2000a] J ürgen Dix, Mirco Nanni, and V S. Subrahmanian. Probabilistic agent reasoning. Transactions of Computational Logic, 1(2):201-245, 2000.

[Dix et al., 2000b] J ürgen Dix, V S. Subrahmanian, and George Pick. Meta Agent Programs. Journal of Logic Programming, 46(1-2):1-60, 2000. 
[Dix et al., 2001a] J"urgen Dix, Ulrich Furbach, and Ilkka Niemel“a. Nonmonotonic Reasoning: Towards Efficient Calculi and Implementations. In Andrei Voronkov and Alan Robinson, editors, Handbook of Automated Reasoning. Elsevier-Science-Press, 2001.

[Dix et al., 2001b] J urgen Dix, Sarit Kraus, and VS Subrahmanian. Temporal agent reasoning. Artificial Intelligence, 127(1):87-135, 2001.

[Dix, 1991] Jürgen Dix. Classifying Semantics of Logic Programs. In Anil Nerode, Wiktor Marek, and V. S. Subrahmanian, editors, Logic Programming and Non-Monotonic Reasoning, Proceedings of the first International Workshop, pages 166-180, Cambridge, Mass., July 1991. Washington D.C, MIT Press.

[Dix, 1992a] Jürgen Dix. A Framework for Representing and Characterizing Semantics of Logic Programs. In B. Nebel, C. Rich, and W. Swartout, editors, Principles of Knowledge Representation and Reasoning: Proceedings of the Third International Conference (KR '92), pages 591-602. San Mateo, CA, Morgan Kaufmann, 1992.

[Dix, 1992b] J ürgen Dix. Classifying Semantics of Disjunctive Logic Programs. In K. R. Apt, editor, LOGIC PROGRAMMING: Proceedings of the 1992 Joint International Conference and Symposium, pages 798-812, Cambridge, Mass., November 1992. MIT Press.

[Dix, 1995a] J urgen Dix. A Classification-Theory of Semantics of Normal Logic Programs: I. Strong Properties. Fundamenta Informaticae, XXII(3):227-255, 1995.

[Dix, 1995b] J ürgen Dix. A Classification-Theory of Semantics of Normal Logic Programs: II. Weak Properties. Fundamenta Informaticae, XXII(3):257-288, 1995.

[Dix, 1995c] Jürgen Dix. Semantics of Logic Programs: Their Intuitions and Formal Properties. An Overview. In Andre Fuhrmann and Hans Rott, editors, Logic, Action and Information - Essays on Logic in Philosophy and Artificial Intelligence, pages 241-327. DeGruyter, 1995.

[Dix, 1998] Jürgen Dix. The Logic Programming Paradigm. AI Communications, Vol. 11, No. 3:3943, 1998. Short version in Newsletter of ALP, Vol. 11(3), 1998, pages 10-14.

[Dix, 2001] J urgen Dix. A Computational Logic Approach to Heterogenous Agent Systems. In Th. Eiter, M. Truszczy 'nski, and W. Faber, editors, Logic Programming and Non-Monotonic Reasoning, Proceedings of the Sixth International Conference, LNCS, Berlin, September 2001. Springer.

[Dowling and Gallier, 1984] W.F. Dowling and J.H. Gallier. Linear Time Algorithms for Testing the Satisfiability of Propositional Horn Formulae. Journal of Logic Programming, 1:267-284, 1984.

[Drabent, 1994] Wlodzimierz Drabent. What is failure? A constructive approach to negation. Acta Informatica, 32(1):27-29, 1994.

[Dung, 1992] P. M. Dung. On the relations between stable and wellfounded semantics of logic programs. Theoretical Computer Science, 105:7-25, 1992.

[Eiter and Gottlob, 1993a] T. Eiter and G. Gottlob. Complexity Aspects of Various Semantics for Disjunctive Databases. In Proceedings of the Twelth ACM SIGACT SIGMOD-SIGART Symposium on Principles of Database Systems (PODS-93), pages 158-167, June 1993.

[Eiter and Gottlob, 1993b] Thomas Eiter and Georg Gottlob. Propositional Circumscription and Extended Closed World Reasoning are $\Pi_{2}^{P}$-complete. Theoretical Computer Science, 144(2):231245, Addendum: vol. 118, p. 315, 1993, 1993.

[Eiter and Gottlob, 1995] Thomas Eiter and Georg Gottlob. The complexity of logic-based abduction. Journal of the ACM, 42(1):3-42, 1995.

[Eiter et al., 1993] Thomas Eiter, Georg Gottlob, and Heikki Mannila. Expressive Power and Complexity of Disjunctive DATALOG. In Proceedings of Workshop on Logic Programming with Incomplete Information, Vancouver Oct. 1993, following ILPS' 93, pages 59-79, 1993.

[Eiter et al., 1997a] T. Eiter, J. Lu, and V. S. Subrahmanian. Computing Non-Ground Representations of Stable Models. In J. Dix, U. Furbach, and A. Nerode, editors, Logic Programming and NonMonotonic Reasoning, Proceedings of the Fourth International Conference, LNAI 1265, pages 198-217, Berlin, July 1997. Springer.

[Eiter et al., 1997b] Thomas Eiter, Nicola Leone, Cristinel Mateis, Gerald Pfeifer, and Francesco Scarcello. A Deductive System for Nonmonotonic Reasoning. In J urgen Dix, Ulrich Furbach, and Anil Nerode, editors, Proceedings of the 4th International Conference on Logic Programming and Nonmonotonic Reasoning (LPNMR '97), number 1265 in Lecture Notes in AI (LNAI), Berlin, 1997. Springer.

[Eiter et al., 1998] Thomas Eiter, Nicola Leone, Cristinel Mateis, Gerald Pfeifer, and Francesco Scarcello. The KR System dlv: Progress Report, Comparisons and Benchmarks. In Proceedings Sixth International Conference on Principles of Knowledge Representation and Reasoning (KR'98), pages 406-417, 1998. 
[Eiter et al., 1999] Thomas Eiter, V. S. Subrahmanian, and Georg Pick. Heterogeneous Active Agents, I: Semantics. Artificial Intelligence, 108(1-2):179-255, 1999.

[Eiter et al., 2000] T. Eiter, V.S. Subrahmanian, and T J. Rogers. Heterogeneous Active Agents, III: Polynomially Implementable Agents. Artificial Intelligence, 117(1):107-167, 2000.

[Fages, 1993] F. Fages. Consistency of Clark's completion and existence of stable models. Methods of Logic in Computer Science, 2, 1993.

[Fern 'andez and Minker, $199 \$$ J. A. Fern'andez and J. Minker. Bottom-Up Computation of Perfect Models for Disjunctive Theories. Journal of Logic Programming, 25(1):33-51, 1995.

[Fern'andez et al., 1993 J. A. Fern 'andez, J. Lobo, J. Minker, and V.S. Subrahmanian. Disjunctive LP + Integrity Constraints $=$ Stable Model Semantics. Annals of Mathematics and Artificial Intelligence, 8(3-4), 1993.

[Fitting, 1985] Melvin C. Fitting. A Kripke-Kleene Semantics of logic Programs. Journal of Logic Programming, 4:295-312, 1985.

[Garey and Johnson, 1979] M.R. Garey and D.S. Johnson. Computers and Intractability. W.H. Freeman and Company, San Francisco, 1979.

[Gelfond and Lifschitz, 1988] Michael Gelfond and Vladimir Lifschitz. The Stable Model Semantics for Logic Programming. In R. Kowalski and K. Bowen, editors, 5th Conference on Logic Programming, pages 1070-1080. MIT Press, 1988.

[Gelfond, 1994] Michael Gelfond. Logic programming and reasoning with incomplete information. Annals of Mathematics and Artificial Intelligence, 12:89-116, 1994.

[Gogic et al., 1995] Goran Gogic, Christos Papadimitriou, Bart Selman, and Henry Kautz. The Comparative Linguistics of Knowledge Representation. In Proceedings of the 14th International Joint Conference on Artificial Intelligence, pages 862-869, Montreal, Canada, August 1995. Morgan Kaufmann Publishers.

[Gurevich, 1988] Y. Gurevich. Logic and the Challenge of Computer Science. In E. B orger, editor, Trends in Theoretical Computer Science, chapter 1. Computer Science Press, 1988.

[Horty et al., 1990] Jeff Horty, Richmond Thomason, and D. S. Touretzky. A skeptical Theory of Inheritance in Nonmonotonic Semantic Networks. Artificial Intelligence, 42:311-348, 1990.

[Imielinski, 1991] T. Imielinski. Incomplete Deductive Databases. Annals of Mathematics and Artificial Intelligence, 3:259-294, 1991.

[Inoue et al., 1992] Katsumi Inoue, M. Koshimura, and R. Hasegawa. Embedding negation-as-failure into a model generation theorem prover. In Deepak Kapur, editor, Automated Deduction - CADE11, number 607 in LNAI, Berlin, 1992. Springer.

[Johnson, 1990] D.S. Johnson. A catalog of complexity classes. In J. van Leeuwen, editor, Handbook of Theoretical Computer Science, volume A. Algorithms and Complexity, pages 67-161. Elsevier, 1990.

[Kagan et al., 1994] Vadim Kagan, Anil Nerode, and V. S. Subrahmanian. Computing Definite Logic Programs by Partial Instantiation. Annals of Pure and Applied Logic, 67:161-182, 1994.

[Kagan et al., 1995] Vadim Kagan, Anil Nerode, and V. S. Subrahmanian. Computing Minimal Models by Partial Instantiation. Theoretical Computer Science, 155:157-177, 1995.

[Kakas et al., 2001] Toni Kakas, Robert Miller, and Francesca Toni. E-res: Reasoning about actions, events and observations. In Proc. LPNMR 2001. Springer, 2001.

[Kemp et al., 1991] David B. Kemp, Peter J. Stuckey, and Divesh Srivastava. Magic Sets and BottomUp Evaluation of Well-Founded Models. In Vijay Saraswat and Kazunori Ueda, editors, Proceedings of the 1991 Int. Symposium on Logic Programming, pages 337-351. MIT, June 1991.

[Konolige, 1988] Kurt Konolige. Hierarchic autoepistemic theories for nonmonotonic reasoning. In Proceedings AAAI '88. Morgan Kaufmann, 1988.

[Kowalski, 1974] R.A. Kowalski. Predicate logic as a programming language. In Proceeedings IFIP' 74, pages 569-574. North Holland Publishing Company, 1974.

[Kunen, 1987] Kenneth Kunen. Negation in Logic Programming. Journal of Logic Programming, 4:289-308, 1987.

[Kunen, 1989] Kenneth Kunen. Signed Data Dependencies. Journal of Logic Programming, 7:231$245,1989$.

[Lifschitz, 1985] Vladimir Lifschitz. Computing Circumscription. In Proceedings of the International Joint Conference on Artificial Intelligence, Los Angeles, California, pages 121-127, 1985.

[Lifschitz, 1994] V. Lifschitz. Circmscription, pages 297-353. Clarendon, Oxford, 1994.

[Lifschitz, 1996] V. Lifschitz. Foundations of declarative logic programming. In G. Brewka, editor, Principles of Knowledge Representation, chapter 3, pages 69-128. CSLI, 1996. 
[Lloyd, 1987] John W. Lloyd. Foundations of Logic Programming. Springer, Berlin, 1987. 2nd edition.

[Lobo et al., 1992] Jorge Lobo, Jack Minker, and Arcot Rajasekar. Foundations of Disjunctive Logic Programming. MIT-Press, 1992.

[Lud“ascher, 1991] Bertram Lud“ascher. CNF-Prolog: A Meta-Interpreter for Chan's Constructive Negation, Implementation. Technical report, Master Thesis, Karlsruhe University (in german), 1991.

[Mancarella et al., 1988] Paolo Mancarella, Simone Martini, and Dino Pedreschi. Complete logic Programs with domain-closure Axiom. Journal of Logic Programming, 5:263-276, 1988.

[Marek and Truszczy'nski, 1993 Wiktor Marek and Mirek Truszczy'nski. Nonmonotonic Logics Context-Dependent Reasoning. Springer, 1993.

[Marek et al., 1992] Wiktor Marek, Arcot Rajasekar, and Mirek Truszczy 'nski. Complexity of Computing with Extended Propositional Logic Programs. In Proc. of the Workshop W1, Structural Complexity and Recursion-theoretic Methods in Logic Programming, following the IJCSLP '92, pages 93-102. H. Blair and W. Marek and A. Nerode and J. Remmel, November 1992.

[McCarthy, 1979] John McCarthy. First order theories of individual concepts and propositions. In B. Meltzer and D. Michie, editors, Machine Intelligence 9, pages 120-147. Edinburgh University Press, Edinburgh, 1979.

[McCarthy, 1980] John McCarthy. Circumscription: A Form of Nonmonotonic Reasoning. Artificial Intelligence, 13:27-39, 1980.

[McCarthy, 1986] John McCarthy. Applications of Circumscription to formalizing Common-Sense Reasoning. Artificial Intelligence, 28:89-116, 1986.

[Minker and Ruiz, 1995] Jack Minker and Carolina Ruiz. Computing stable and partial stable models of extended disjunctive logic programs. In J. Dix, L. Pereira, and T. Przymusinski, editors, Nonmonotonic Extensions of Logic Programming, LNAI 927, pages 205-229. Springer, Berlin, 1995.

[Minker, 1982] Jack Minker. On indefinite databases and the closed world assumption. In Proceedings of the 6th Conference on Automated Deduction, New York, pages 292-308, Berlin, 1982. Springer.

[Minker, 1988] Jack Minker. Foundations of Deductive Databases. Morgan Kaufmann, 95 First Street, Los Altos, CA 94022, 1st edition, 1988.

[Minker, 1993] Jack Minker. An Overview of Nonmonotonic Reasoning and Logic Programming. Journal of Logic Programming, Special Issue, 17(2/3/4):95-126, 1993.

[Minker, 1996] Jack Minker. Logic and databases: A 20 year retrospective. In Dino Pedreschi and Carlo Zaniolo, editors, Proceedings of the International Workshop on Logic in Databases (LID), LNCS 1154, pages 3-58. Springer, Berlin, 1996.

[Moschovakis, 1974] Y. N. Moschovakis. Elementary Induction on Abstract Structures. NorthHolland, 1974.

[Müller and Dix, 1993 Martin Müller and Jürgen Dix. Implementing Semantics for Disjunctive Logic Programs Using Fringes and Abstract Properties. In Luis Moniz Pereira and Anil Nerode, editors, Logic Programming and Non-Monotonic Reasoning, Proceedings of the Second International Workshop, pages 43-59, Cambridge, Mass., July 1993. Lisbon, MIT Press.

[Nebel, 1998] Bernhard Nebel. How hard is it to revise a belief base? In Didier Dubois and Henri Prade, editors, Handbook on Defeasible Reasoning and Uncertainty Management Systems, pages 77-145. Kluwer Academic, 1998.

[Nerode et al., 1991] Anil Nerode, Raymond T. Ng, and V.S. Subrahmanian. Computing Circumscriptive Deductive Databases. CS-TR 91-66, Computer Science Dept., Univ. Maryland, University of Maryland, College Park, Maryland, 20742, USA, December 1991.

[Niemel“a and Simons, 1996 Ilkka Niemel“a and Patrik Simons. Efficient Implementation of the Wellfounded and Stable Model Semantics. In M. Maher, editor, Proceedings of the Joint International Conference and Symposium on Logic Programming, pages 289-303, Bonn, Germany, September 1996. The MIT Press.

[Niemel“a, 1996a Ilkka Niemel"a. Implementing circumscription using a tableau method. In W. Wahlster, editor, Proceedings of the European Conference on Artificial Intelligence, pages 8084, Budapest, Hungary, August 1996. John Wiley.

[Niemel“a, 1996b Ilkka Niemel”a. A tableau calculus for minimal model reasoning. In P. Miglioli, U. Moscato, D. Mundici, and M. Ornaghi, editors, Proceedings of the Fifth Workshop on Theorem Proving with Analytic Tableaux and Related Methods, pages 278-294, Terrasini, Italy, May 1996. LNAI 1071, Springer-Verlag. 
[Niemel“a, 1999 Ilkka Niemel“a. Logic Programs with Stable Model Semantics as a Constraint Programming Paradigm. Annals of Mathematics and Artificial Intelligence, Special Issue on Logics in Artificial Intelligence, edited by J. Dix and J. Lobo, 25(3-4):241-273, 1999.

[Odifreddi, 1989] P. Odifreddi. Classical Recursion Theory. North-Holland, 1989.

[Papadimitriou, 1994] C.H. Papadimitriou. Computational Complexity. Addison-Wesley, 1994.

[Pereira and Alferes, 1992] L.M. Pereira and J.J. Alferes. Well founded semantics for logic programs with explicit negation. In Bernd Neumann, editor, Proc. of 10th European Conf. on Artificial Intelligence ECAI 92, pages 102-106. John Wiley \& Sons, 1992.

[Pereira et al., 1993] L. M. Pereira, J. N. Aparício, and J. J. Alferes. Non-Monotonic Reasoning with Logic Programming. Journal of Logic Programming, 17:227-264, 1993.

[Poole, 1985] D. Poole. On the comparison of theories: Preferring the most specific explanation. In Proc. IJCAI-85, Los Angeles, 1985.

[Prakken, 1993] H. Prakken. Logical Tools for Modelling Legal Argument. PhD thesis, VU Amsterdam, 1993.

[Przymusinski, 1991] Teodor Przymusinski. Stationary Semantics for Normal and Disjunctive Logic Programs. In C. Delobel, M. Kifer, and Y. Masunaga, editors, DOOD '91, Proceedings of the 2nd International Conference, Berlin, December 1991. Muenchen, Springer. LNCS 566.

[Przymusinski, 1995] Teodor Przymusinski. Static Semantics For Normal and Disjunctive Logic Programs. Annals of Mathematics and Artificial Intelligence, 14:323-357, 1995.

[Rajasekar et al., 1989] Arcot Rajasekar, Jorge Lobo, and Jack Minker. Weak Generalized Closed World Assumption. Journal of Automated Reasoning, 5:293-307, 1989.

[Reiter, 1978] R. Reiter. On Closed-World Databases. In H. Gallaire and J. Minker, editors, Logic and Data Bases, pages 55-76. Plenum Press, New York, 1978.

[Reiter, 1980] Raymond Reiter. A Logic for Default-Reasoning. Artificial Intelligence, 13:81-132, 1980.

[Ross and Topor, 1988] Kenneth A. Ross and Rodney A. Topor. Inferring negative Information from disjunctive Databases. Journal of Automated Reasoning, 4:397-424, 1988.

[Ross, 1989] Kenneth A. Ross. The well-founded semantics for disjunctive logic programs. In Proceedings of the first International Conference on Deductive and Object Oriented Databases, Kyoto, Japan, pages 1-22, 1989.

[Russel and Norvig, 1995] Stuart Russel and Peter Norvig. Artificial Intelligence - A Modern Approach. Prentice Hall, New Jersey 07458, 1995.

[Sacca, 1993] Domenico Sacca. The Expressive Power of Stable Models For DATALOG Queries with Negation. In Proceedings of Workshop on Logic Programming with Incomplete Information, Vancouver Oct. 1993, following ILPS' 93, pages 150-162, 1993.

[Sakama and Inoue, 1993] Chiaki Sakama and Katsumi Inoue. Negation in Disjunctive Logic Programs. In D. Warren and Peter Szeredi, editors, Proceedings of the 10th Int. Conf. on Logic Programming, Budapest, pages 703-719, Cambridge, Mass., July 1993. MIT Press.

[Sakama and Inoue, 1994] Ch. Sakama and K. Inoue. An Alternative Approach to the Semantics of Disjunctive Logic Programs and Deductive Databases. Journal of Automated Reasoning, 13:145172,1994

[Sakama and Seki, 1994] Chiaki Sakama and Hirohisa Seki. Partial Deduction of Disjunctive Logic Programs: A Declarative Approach. In Logic Program Synthesis and Transformation - Meta Programming in Logic, LNCS 883, pages 170-182, Berlin, 1994. Springer.

[Sakama and Seki, 1997] Chiaki Sakama and Hirohisa Seki. Partial Deduction in Disjunctive Logic Programming. Journal of Logic Programming, 32(3):229-245, 1997.

[Sakama, 1989] Chiaki Sakama. Possible Model Semantics for Disjunctive Databases. In Won Kim, Jean-Marie Nicolas, and Shojiro Nishio, editors, Deductive and Object-Oriented Databases, Proceedings of the First International Conference (DOOD89), pages 369-383, Kyoto, Japan, 1989. North-Holland Publ.Co.

[Schaub and Wang, 2001] T. Schaub and K. Wang. A comparative study of logic programs with preference: Preliminary report. In A. Provetti and S. Cao, editors, Proceedings of AAAI Spring Symposium on Answer Set Programming, pages 151-157. AAAI Press, 2001.

[Schlipf, 1990] John S. Schlipf. The Expressive Powers of the Logic Programming Semantics. In Proceedings of the Ninth ACM Symposium on Principles of Databases, pages 196-204, 1990. 
[Schlipf, 1992] John S. Schlipf. A Survey of Complexity and Undecidability Results in Logic Programming. In H. Blair, W. Marek, A. Nerode, and J. Remmel, editors, Proceedings of the Workshop on Complexity and Recursion-theoretic Methods in Logic Programming, following the JICSLP'92. informal, 1992.

[Shepherdson, 1988] John C. Shepherdson. Negation in Logic Programming. In Jack Minker, editor, Foundations of Deductive Databases, chapter 1, pages 19-88. Morgan Kaufmann, 1988.

[Shepherdson, 1991] John C. Shepherdson. Unsolvable Problems for SLDNF-Resolution. Journal of Logic Programming, 10:19-22, 1991.

[Shepherdson, 1992] John C. Shepherdson. SLDNF-Resolution with Equality. Journal of Automated Reasoning, 8, No. 2:297-306, 1992.

[St"ark, 1994 Robert F. St äk. Input/output dependencies of normal logic programs. Journal of Logic and Computation, 4(3):249-262, 1994.

[Subrahmanian et al., 2000] V.S. Subrahmanian, Piero Bonatti, J ürgen Dix, Thomas Eiter, Sarit Kraus, Fatma Özcan, and Robert Ross. Heterogenous Active Agents. MIT-Press, 2000.

[Swift, 1999] Terry Swift. Using Tabling for nonmonotonic Programming. Annals of Mathematics and Artificial Intelligence, Special Issue on Logics in Artificial Intelligence, edited by J. Dix and J. Lobo, 25(3-4):201-240, 1999.

[Tamaki and Sato, 1986] H. Tamaki and T. Sato. OLD Resolution with Tabulation. In Proceedings of the Third International Conference on Logic Programming, London, LNAI, pages 84-98, Berlin, June 1986. Springer.

[Tarski, 1955] A. Tarski. A lattice-theoretical fixpoint theorem and its applications. Pacific Journal of Mathematics, 5:285-309, 1955.

[Touretzky et al., 1988] David S. Touretzky, Jeff Horty, and Richmond Thomason. A Clash of Intuitions: The current State of Nonmonotonic Multiple IHS. In Proceedings IJCAJ, 1988.

[Touretzky et al., 1991] D. S. Touretzky, R. H. Thomason, and J. F. Horty. A skeptic's menagerie: Confictors, preemptors, reinstaters, and zombies in nonmonotonic inheritance. In Proc. 12th IJCAI, Sydney, 1991.

[Touretzky, 1986] D. S. Touretzky. The Mathematics of Inheritance. Research Notes in Artificial Intelligence. Pitman, London, 1986.

[Ullman, 1989a] J. D. Ullman. Principles of Database and Knowledge Base Systems. Computer Science Press, 1989.

[Ullman, 1989b] Jeffrey D. Ullman. Bottom-up Beats Top-down for Datalog. In Proc. of the Eight ACM SIGACT-SIGMOD-SIGART Symposium on Principles of Database Systems, Philadelphia, Pennsylvania, pages 140-149. ACM Press, March 1989.

[van Emden and Kowalski, 1976] M.H. van Emden and R.A. Kowalski. The semantics of predicate logic as a programming language. JACM, 23:733-742, 1976.

[Van Gelder et al., 1988] Allen Van Gelder, Kenneth A. Ross, and J. S. Schlipf. Unfounded Sets and well-founded Semantics for general logic Programs. In Proceedings 7th Symposion on Principles of Database Systems, pages 221-230, 1988.

[Vorbeck, 1991] Martin Vorbeck. CNF-Prolog: A Meta-Interpreter for Chan's Constructive Negation, Theory. Technical report, Master Thesis, Karlsruhe University (in german), 1991.

[Witteveen, 1991a] Cees Witteveen. Partial Semantics for Truth Maintenance. In J. van Eijck, editor, Logics in AI, LNAI 478, Berlin, 1991. Springer.

[Witteveen, 1991b] Cees Witteveen. Skeptical Reason Maintenance is Tractable. In J. Allen, R. Fikes, and B. Sandewall, editors, Proceedings of the second Conference on Principles of Knowledge Representation and Reasoning, Cambridge, Massachusetts, pages 570-581. Morgan Kaufmann, 1991.

\section{A APPENDIX}

\section{A.1 Predicate Logic}

We assume the reader is familiar with the basic notions of predicate logic such as models, formulae, satisfi ability $\models$ and derivability $\vdash$. There exist several calculi for fi rst-order predicate logic like Hibert-style, Resolution-style, Gentzen-style or 
natural deduction-style calculi. One of the main theorems states the completeness of such calculi with respect to the semantics given by models:

Theorem A.1 (Completeness).

A formula $\varphi$ follows semantically from a theory $T$ (is true in all models of $T$ ) iff $\varphi$ is derivable from $T$ by means of a particular calculus.

$$
T \vdash \varphi \text { iff } T \models \varphi
$$

This theorem tells us that we can enumerate all the theorems of a theory, but it does not provide us with a decision-method to do so. In fact, as we will explain now, such a method does not exist.

Before turning to undecidability, let us emphasize that in the whole paper we are

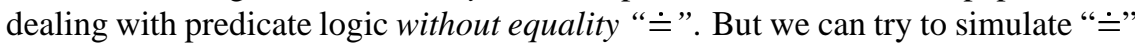
as follows. We introduce a binary relation-symbol $e q$ and require that it satisfi es the following axioms with respect to an underlying language $\mathcal{L}$ :

$$
\forall x e q(x, x)
$$

for all function-symbols $f$ of suitable arity:

$$
\begin{aligned}
& \forall x_{1} \ldots x_{n}, y_{1} \ldots y_{n} \\
& \left(e q\left(x_{1}, y_{1}\right) \ldots e q\left(x_{n}, y_{n}\right)\right) \rightarrow e q\left(f\left(x_{1}, \ldots, x_{n}\right), f\left(y_{1}, \ldots, y_{n}\right)\right)
\end{aligned}
$$

for all predicate-symbols $P$ of suitable arity:

$$
\begin{aligned}
& \forall x_{1} \ldots x_{n} y_{1} \ldots y_{n} \\
& \left(e q\left(x_{1}, y_{1}\right) \ldots e q\left(x_{n}, y_{n}\right)\right) \rightarrow\left(P\left(x_{1}, \ldots, x_{n}\right) \rightarrow P\left(y_{1}, \ldots, y_{n}\right)\right) .
\end{aligned}
$$

This set, is denoted by $\mathrm{EQ}_{\mathcal{L}}$. It can be shown that transitivity and symmetry of $e q$ follow from these axioms. Let us consider the language of Arithmetic $\mathcal{L}_{A r}$ which contains: 0 (a constant), $s$ (a unary function-symbol), $e q$ (a two-ary relationsymbol) and $\oplus, \otimes$ (ternary relation-symbols).

We have in mind to axiomatize the theory of natural numbers. Before we do so we introduce the following abbreviation. The formula $\exists ! z \phi(z)$ stands for

$$
\exists z(\phi(z) \wedge \forall y \phi(y) \rightarrow e q(y, z)) .
$$

Definition A.1 (Arithmetic $\mathbf{A r}_{\text {fin }}$ ).

$\operatorname{Ar}_{f i n}$ is the fi nite set consisting of $\mathrm{EQ}_{\mathcal{L}_{A r}}$ and the following axioms:

$$
\begin{array}{ll}
\forall x \forall y \exists ! z & \oplus(x, y, z) \\
\forall x & \oplus(x, 0, x) \\
\forall x \forall y \forall z & \oplus(x, y, z) \rightarrow \oplus(x, s(x), s(z))
\end{array}
$$

$\forall x \forall y \exists ! z \quad \otimes(x, y, z)$

$\forall x \quad \otimes(x, 0,0)$

$\forall x \forall y \forall z \forall z^{\prime} \quad \otimes(x, y, z) \rightarrow\left(\otimes\left(x, s(y), z^{\prime}\right) \wedge \oplus\left(z, x, z^{\prime}\right)\right)$ 
The set of natural numbers $\mathcal{N}:=\left(\mathbb{N}, 0^{\mathcal{N}}, s^{\mathcal{N}}, \oplus^{\mathcal{N}}, \otimes^{\mathcal{N}}, e q^{\mathcal{N}}\right)$ is a model of $\operatorname{Ar}_{\text {fin }}$. Here $0^{\mathcal{N}}$ is the "true" $0, s^{\mathcal{N}}$ is the successor-function, $\oplus^{\mathcal{N}}$ is addition and $\otimes^{\mathcal{N}}$ is multiplication (viewed as relations), $e q^{\mathcal{N}}$ is identity. We note the following facts:

1. The set $\left\{\phi: A r_{\text {fin }} \models \phi\right\}$ is recursively enumerable but not recursive.

2. The set $\{\phi: \mathcal{N} \models \phi\}$ is not even recursively enumerable.

We even have

\section{Theorem A.2 (Gödel).}

No set of formulae containing $A r_{\text {fin }}$ and having $\mathcal{N}$ as model, is recursive.

Every recursively enumerable set of formulae $\Phi$ that contains $A r_{f i n}$ and has $\mathcal{N}$ as a model, is incomplete, i.e. there is $\psi$ with: $\mathcal{N} \models \psi$ but $\Phi \not \forall \psi$. Therefore no complete axiomatization of $\mathcal{N}$ is possible.

Note that, although $\mathcal{N}$ formally is not a Herbrand model, it is isomorphic to such a model. In fact, the axioms immediately imply that there is, up to isomorphy, only one single Herbrand-model of $\operatorname{Ar}_{f i n}$ with respect to $\mathcal{L}_{A r}$. Therefore to determine if a formula is true in all Herbrand-models of $\mathrm{Ar}_{f i n}$ is just as complicated as the theory of $\mathcal{N}$ itself. $\mathcal{N}$ contains, for example, famous statements (or there negation) from number theory like Goldbach-conjecture or Fermat's last theorem.

\section{A.2 Complexity Theory}

We assume some familiarity with the classes $\mathrm{P}$ (problems solvable in deterministic polynomial time) and $\mathrm{NP}$ (problems solvable in nondeterministic polynomial time). The class co-NPis the complement of NP, i.e. a problem is in co-NPif its complement is in NP. From these sets we can build larger classes by considering problems solvable in deterministic (resp. nondeterministic) time where we allow to ask queries to an NP-oracle: i.e. whenever we come up with a subproblem that lies in NP, we just ask an oracle which immediately gives us the answer (we count this as just one step). This gives rise to the polynomial hierarchy:

\section{Definition A.2 (Polynomial Hierarchy).}

For a complexity class $C$ we denote by $\mathrm{P}^{C}$ (resp. $\mathrm{NP}^{C}$ ) the class of problems solvable in deterministic polynomial (resp. nondeterministic polynomial) time using $C$-oracles. Let $\Sigma_{0}:=\Pi_{0}:=\mathrm{P}$ and

$$
\begin{aligned}
& \Sigma_{k+1}:=\mathrm{NP}^{\Sigma_{k}} \\
& \Pi_{k+1}:=\mathrm{co}-\mathrm{NP}^{\Sigma_{k}} \\
& \Delta_{k+1}:=\mathrm{P}^{\Sigma_{k}}
\end{aligned}
$$

Thus $\Sigma_{1}$ is NPwith queries to a P-oracle, i.e. $\Sigma_{1}=$ NP. Similarly we have $\Pi_{1}=$ co-NP and $\Delta_{1}=\mathrm{P}$. A problem is in $\Delta_{2}=\mathrm{P}^{\mathrm{NP}}$ if it can be solved in deterministic polynomial time with subcalls to an NP-oracle. Although the index is $2, \Delta_{2}$ is considered to belong to the fi rst level of the polynomial hierarchy. 
The second level of this hierarchy consists of $\Sigma_{2}, \Pi_{2}$ and $\Delta_{3}$. Here $\Sigma_{2}:=$ $\mathrm{NP}^{\mathrm{NP}}$ : nondeterministic polynomial time with queries to an NP-oracle. $\Pi_{2}:=$ co-NP ${ }^{\mathrm{NP}}$ and $\left.\Delta_{3}:=\mathrm{P}^{[\mathrm{NP}}\right]$.

It is immediate that

$$
\Sigma_{k} \cup \Pi_{k} \subseteq \Delta_{k+1} \subseteq \Sigma_{k+1} \cap \Pi_{k+1}
$$

but it has not yet been proved that the inclusions are proper. That is, it is not known if the hierarchy collapses at some point or not.

The polynomial hierarchy classifi es a subclass of all decidable problems, namely those that are NP-hard. A problem is called NP-hard if any other problem in NPcan be polynomially reduced to it. Of particular interest are those problems in a class $\Pi_{k}$ or $\Sigma_{k}$ that are the hardest ones: they are called complete. This means that all problems in the respective class can be polynomially reduced to such a complete problem and the problem itself belongs to this class. As an example, to determine if a formula is valid is co-NP-complete. Thus, satisfi ability of a propositional formula is NP-complete.

An analogue hierarchy exists (in fact it was the prototype of the polynomial hierarchy) for undecidable problems. The notation is analog to the one just introduced. Therefore one often adds a superscript $P$ to the $\Pi_{k}$ and $\Sigma_{k}$ which stands for polynomial (but not for an oracle) to denote the polynomial hierarchy.

To introduce the arithmetical hierarchy we consider the model $\mathcal{N}$ of the natural numbers and $\mathcal{L}_{A r}$-formulae. We call such formulae for short arithmetical. We classify arithmetical formulae according to their quantifi er-alternations:

\section{Definition A.3 (Arithmetical Hierarchy).}

We call an arithmetical formula $\Sigma_{k}^{0}$ (resp. $\Pi_{k}^{0}$ ) if it is of the form $\exists \forall \ldots \phi$ (resp. $\forall \exists \ldots \phi$ ) where $\phi$ is quantifi er-free and there are at most $k-1$ alternations of quantifi erblocks.

We call a set $M$ of natural numbers $\Sigma_{k}^{0}$-defi nable, if $M$ is defi nable by a $\sum_{k}^{0}$ formula. This means that there is a $\Sigma_{k}^{0}$-formula $\phi(x)$ with one free variable $x$ such that

$$
\mathcal{N} \models \phi(i) \text { iff } i \in M \text {. }
$$

Note that the $\Sigma_{0}^{0}$-defi nable sets coincide with the $\Pi_{0}^{0}$-defi nable ones: they are exactly the recursive sets. The recursive enumerable sets are the $\Sigma_{1}^{0}$-defi nable ones, the $\Pi_{1}^{0}$-defi nable sets are their complements. The set corresponding to the famous Halting Problem, i.e. the set of all Gödel numbers of those Turing-machines that stop on their own Gödel number, is $\Sigma_{1}^{0}$, so this problem is located very low in the hierarchy.

The higher a problem lies in the hierarchy, the more undecidable it is. For example a problem located at the second level, say $\Sigma_{2}^{0}$, can be thought of as being recursively enumerable using an oracle which solves $\Sigma_{1}^{0}$-problems (like the halting problem). 
Analogously to the polynomial hierarchy we have the notions of $\Sigma_{k}^{0}$-complete and $\Pi_{k}^{0}$-complete. As an example, the halting problem is $\Sigma_{1}^{0}$-complete.

In contrast to the polynomial hierarchy, the arithmetical hierarchy is strict. We denote by $\Delta_{k}^{0}$ the intersection of $\Sigma_{k+1}^{0}$ and $\Pi_{k+1}^{0}$. We have

$$
\Sigma_{k}^{0} \cup \Pi_{k}^{0} \subset \Delta_{k}^{0}=\Sigma_{k+1}^{0} \cap \Pi_{k+1}^{0} .
$$

Are there more undecidable problems, not yet captured by our hierarchy? Yes, take for example the theory of $\mathcal{N}$ considered in Section A.1. Obviously, the general problem to determine if an arbitrary formula is true or not in $\mathcal{N}$ can not be captured at a certain level, because the class of formulae in question can have unlimited alternations of quantifi ers. The careful reader may have asked himself what the superscript 0 means in $\Sigma_{k}^{0}$ ? It just means that we consider just fi rst-order formulae and we do not allow our arithmetical formulae to contain second-order quantifi ers.

This remark gives rise to the analytical hierarchy, denoted by $\Sigma_{k}^{1}, \Pi_{k}^{1}$, where we consider second-order arithmetical formulae. We only count the alternations of the quantifi ers over sets. So any $\Sigma_{k}^{0}$-formula is in $\Sigma_{0}^{1}$.

Note that for the arithmetical hierarchy the identity $\Sigma_{0}^{0}=\Sigma_{1}^{0} \cap \Pi_{1}^{0}$ holds. The analogue for the analytical hierarchy does not hold. A counterexample is given by the theory of the natural numbers $\mathcal{N}$ : the set of true sentences in arithmetic is in $\Sigma_{1}^{1} \cap \Pi_{1}^{1}$ but not in $\Sigma_{0}^{1}$. This set is also called hyperarithmetical for obvious reasons.

For a more detailed treatment of the topics in this section we refer the reader to the standard literature: [Balcázar et al., 1988; Garey and Johnson, 1979; Johnson, 1990] and [Papadimitriou, 1994; Odifreddi, 1989] for undecidability.

\section{A.3 Default Logic}

Reiter's default logic [Reiter, 1980] is one of the most prominent nonmonotonic logics. Default logic assumes knowledge to be represented in terms of a default theory. A default theory is a pair $(D, W)$. W is a set of first order formulae representing the facts which are known to be true with certainty. $D$ is a set of defaults the form

$$
\frac{A: B_{1}, \ldots, B_{n}}{C}
$$

where $A, B_{i}$ and $C$ are classical formulae. We will also frequently use the alternative, less space consuming notation $A: B_{1}, \ldots, B_{n} / C$ for this default. The default has the intuitive reading: if $A$ is provable and, for all $i(1 \leq i \leq n), \neg B_{i}$ is not provable, then derive $C$. $A$ is called the prerequisite, $B_{i}$ a consistency condition or justifi cation, and $C$ the consequent of the default. For a default $d$ we use pre $(d)$, $j u s t(d)$, and cons $(d)$ to denote the prerequisite, the set of justifi cations, and the consequent of $d$, respectively. Open defaults, i.e., defaults with free variables, are usually interpreted as schemata representing all of their closed instances. ${ }^{19}$

\footnotetext{
${ }^{19}$ Reiter treats open defaults somewhat differently and uses a more complicated method to define extensions for them.
} 
Default theories induce so-called extensions which represent acceptable belief sets a reasoner may adopt based on the available information. A formula $p$ is called a skeptical consequence of $(D, W)$ iff $p$ is contained in all extensions of $(D, W)$. $p$ is called a credulous consequence of $(D, W)$ iff $p$ is contained in at least one extension of $(D, W)$.

We will fi rst present a defi nition of extensions which is slightly different from (but equivalent to) Reiter's original defi nition. We have found that this defi nition is somewhat easier to digest. The original defi nition will be presented later.

Intuitively, $E$ is an extension of $(D, W)$ iff $E$ is a deductively closed (in the sense of classical logic) superset of $W$ satisfying the following two properties

1. all defaults that are "applicable" with respect to $E$ have been applied,

2. every formula in $E$ has a "derivation" from $W$ and applicable defaults.

To make the two requirements more precise we introduce the following notion:

\section{Definition A.4 (Default Proof).}

Let $(D, W)$ be a default theory, $S$ a set of formulae, and $p$ a formula. A $(D, W)$ default proof for $p$ is a fi nite sequence $P=\left(d_{1}, \ldots, d_{n}\right)$ of defaults in $D$ such that:

1. $W \cup\left\{\operatorname{cons}\left(d_{1}\right), \ldots, \operatorname{cons}\left(d_{i-1}\right)\right\} \vdash \operatorname{pre}\left(d_{i}\right)$, for $i \in\{1, \ldots, n\}$,

2. $W \cup\left\{\operatorname{cons}\left(d_{1}\right), \ldots, \operatorname{cons}\left(d_{n}\right)\right\} \vdash p$.

$P$ is valid in $S$ iff $S$ does not contain the negation of a justifi cation of a default in $P$.

As usual $\vdash$ denotes classical provability. We now can state the defi nition of extensions formally:

Definition A.5 (Extension 1).

Let $(D, W)$ be a default theory. $E$ is an extension of $(D, W)$ iff $E$ is a deductively closed superset of $W$ satisfying the conditions

1. if $A: B_{1}, \ldots, B_{n} / C \in D, A \in E$ and for all i $(1 \leq i \leq n) \neg B_{i} \notin E$, then $C$ in $E$, and

2. $p \in E$ implies there is a $(D, W)$-default proof for $p$ valid in $E$.

Reiter's equivalent original defi nition is more compact. It defi nes extensions as fi xpoints of a certain operator.

\section{Definition A.6 (Extension 2).}

Let $(D, W)$ be a default theory, $S$ a set of formulae. Let $\Gamma(S)$ be the smallest set such that:

1. $W \subseteq \Gamma(S)$, 
2. $\operatorname{Th}(\Gamma(S))=\Gamma(S)$,

3. if $A: B_{1}, \ldots, B_{n} / C \in D, A \in \Gamma(S), \neg B_{i} \notin S(1 \leq i \leq n)$, then $C \in \Gamma(S)$.

$E$ is an extension of $(D, W)$ iff $E=\Gamma(E)$, that is, if $E$ is a fi xpoint of $\Gamma$.

We fi nally give a third, quasi-inductive characterization of extensions, also due to Reiter. This version is often used in proofs about default logic and makes the way in which formulae have to be grounded in the premises more explicit. Let $E$ be a set of formulae and defi ne, for a given default theory $(D, W)$, a sequence of sets of formulae as follows:

$$
\begin{aligned}
& E_{0}=W, \text { and for } i \geq 0 \\
& E_{i+1}=T h\left(E_{i}\right) \cup\left\{C \mid A: B_{1}, \ldots, B_{n} / C \in D, A \in E_{i}, \neg B_{i} \notin E\right\} .
\end{aligned}
$$

It can be shown that $E$ is an extension of $(D, W)$ iff $E=\bigcup_{i=0}^{\infty} E_{i}$. The appearance of $E$ in the defi nition of $E_{i+1}$ is what renders this alternative defi nition of extensions non-constructive.

Default theories may have an arbitrary number of extensions (including zero). Extensions are always consistent if $W$ is and if there are no degenerate defaults without consistency conditions. If $W$ is inconsistent then the single extension of $(D, W)$ is the set of all formulae. Extensions are maximal in the following sense: if $E$ is an extension then there is no extension $E^{\prime}$ such that $E^{\prime} \subset E$.

\section{A.4 Circumscription}

Circumscription is a method of computing the closure of a theory by restricting its models to those that have minimal extensions of some of the predicates and functions. Since its fi rst formulation by McCarthy [McCarthy, 1980], it has taken on several different forms, including domain circumscription [McCarthy, 1979] (minimizing the elements in the universe of models), and the most popular and useful version, parallel predicate circumscription [McCarthy, 1980; McCarthy, 1986; Lifschitz, 1985] which we present here.

Although circumscription was originally presented as a schema for adding more formulae to a theory (just as Clark's completion does), here we describe it in terms of restricting the models of the theory. This view leads to the generalization of circumscription by model preference theories, and is more useful analytically in relating circumscription to other nonmonotonic formalisms. More detailed references to circumscription can be found in Lifschitz' excellent survey article [Lifschitz, 1994].

Choose a language $\mathcal{L}$, and let $P$ be the set of predicate symbols that we are interested in minimizing, and $Z$ another set of predicate symbols whose interpretation we allow to vary across compared models. For example, if we wish to minimize the number of cannibals, we would let $P=\{C\}$, and $Z$ be all other predicate symbols (the importance of $Z$ will be indicated later). Suppose $A$ is a theory containing the statements $C\left(p_{1}\right), C\left(p_{2}\right)$, and $C\left(p_{3}\right)$, but no other assertions 


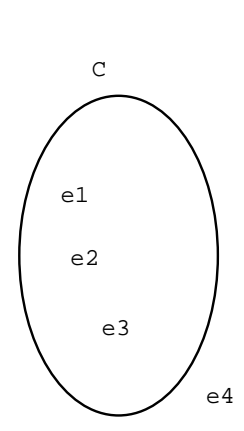

Model M1

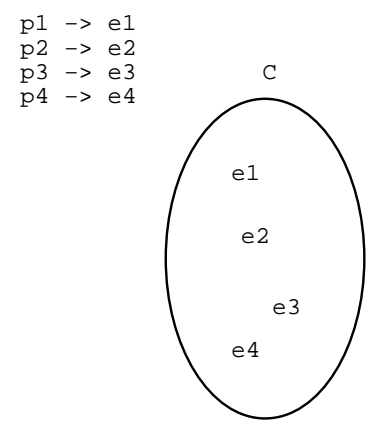

Model M2

Figure 5. Two Models of a Theory with the Same Valuation.

using $C$. Then every model of $A$ will have at least the individuals referred to by $p_{1}, p_{2}$, and $p_{3}$ with property $C$. Now consider two models with the same valuation function from terms to individuals, as in Figure 5. In model $M_{1}$, the extension of the predicate $C$ includes just the three individuals $e_{1}, e_{2}$, and $e_{3}$. In model $M_{2}$ there is a fourth individual, $e_{4}$, who is a cannibal. Circumscription would prefer $M_{1}$ to $M_{2}$, since the extension of $C$ in $M_{1}$ is a proper subset of its extension in $M_{2}$. Under appropriate assumptions (that these terms refer to different individuals), circumscription would yield the result $\neg C\left(p_{4}\right)$, which is not present in the original theory.

Let $A(P, Z)$ be a fi rst-order sentence containing the symbols $P$ and $Z$. Circumscription prefers models of $A(P, Z)$ that are minimal in the predicates $P$, assuming that these models have the same interpretation for all symbols not in $P$ or $Z$. A may contain predicates other than $P$ and $Z$; these are called the fixed symbols.

To state this more formally, let $M_{1}$ and $M_{2}$ be two models of $A(P, Z) .|M|$ is the universe of model $M$, and $M \llbracket K \rrbracket$ is the interpretation of the symbol $K$ in $M$.

Then

\section{Definition A.7 (Minimal Models).}

$M_{1} \leq^{P ; Z} M_{2}$ iff $\begin{cases}1 . & \left|M_{1}\right|=\left|M_{2}\right| . \\ 2 . & M_{1} \llbracket K \rrbracket=M_{2} \llbracket K \rrbracket \text { for all } K \text { not in } P, Z . \\ 3 . & M_{1} \llbracket P_{i} \rrbracket \subseteq M_{2} \llbracket P_{i} \rrbracket \text { for all } P_{i} \in P .\end{cases}$

$\leq^{P ; Z}$ is a preorder relation (reflexive and transitive) on models, but not necessarily a partial order, since it is not antireflexive. We defi ne the strict order $M_{1}<{ }^{(P ; Z)} M_{2}$ as $M_{1} \leq^{P ; Z} M_{2}$ and not $M_{2} \leq^{P ; Z} M_{1}$. The preferred models of $A(P, Z)$ are those that are minimal according to the strict ordering. 